Article

\title{
A Novel Approach to Decision-Making with Pythagorean Fuzzy Information
}

\author{
Sumera $\mathrm{Naz}^{1}$, Samina Ashraf ${ }^{2}$ and Muhammad Akram ${ }^{1, *}$ (D) \\ 1 Department of Mathematics, University of the Punjab, Quaid-e-Azam Campus, Lahore 54590, Pakistan; \\ nsumeranaz@gmail.com \\ 2 Quality Enhancement Cell, Lahore College for Women University, Lahore 54600, Pakistan; \\ saminaa561@gmail.com \\ * Correspondence: m.akram@pucit.edu.pk
}

Received: 6 May 2018; Accepted: 1 June 2018; Published: 7 June 2018

\begin{abstract}
A Pythagorean fuzzy set (PFS) is a powerful tool for depicting fuzziness and uncertainty. This model is more flexible and practical as compared to an intuitionistic fuzzy model. This paper proposes a new graph, called Pythagorean fuzzy graph (PFG). We investigate some properties of our proposed graphs. We determine the degree and total degree of a vertex of PFGs. Furthermore, we present the concept of Pythagorean fuzzy preference relations (PFPRs). In particular, we solve decision-making problems, including evaluation of hospitals, partner selection in supply chain management, and electronic learning main factors evaluation by using PFGs.
\end{abstract}

Keywords: Pythagorean fuzzy graph; Pythagorean fuzzy preference relation; decision making

MSC: 03E05

\section{Introduction}

Since Zadeh's seminal work [1], the classical logic has been extended to fuzzy logic, which is characterized by a membership function in $[0,1]$ and provides a powerful alternative to probability theory to characterize imprecision, uncertainty, and obscureness in various fields. Gradually, it has been discovered that sometimes the membership function of the fuzzy set is not enough to reveal the characters of things because of the complexity of data and the ambiguity of the human mind. To overcome this shortcoming of the fuzzy set, Atanassov [2] extended fuzzy set to an intuitionistic fuzzy set (IFS) by adding a non-membership function and a hesitancy function. An IFS is able to describe the things from three aspects of superiority, inferiority and hesitation, which are usually represented by the intuitionistic fuzzy numbers (IFNs) [3]. To capture more useful information under imprecise and uncertain circumstances, Yager [4-6] recently proposed the concept of Pythagorean fuzzy set (PFS) as a new evaluation format, which is characterized by the membership and the non-membership degree satisfying the condition that their square sum is not greater than 1 . Zhang and $\mathrm{Xu}$ [7] provided the detailed mathematical expression for PFS and put forward the concept of Pythagorean fuzzy number (PFN). The PFS is more general than the IFS because the space of PFS's membership degree is greater than the space of IFS's membership degree. For instance, when a decision-maker gives the evaluation information whose membership degree is 0.4 and non-membership degree is 0.9 , it can be known that the IFN fails to address this issue because $0.4+0.9>1$. However, $(0.4)^{2}+(0.9)^{2}<1$, that is to say, the PFN is capable of representing this evaluation information, as shown in Figure 1. For this case, the PFS shows its wider applicability than the IFS. PFSs as a novel evaluation have been prosperously applied in various fields, such as the internet stocks investment [8], the service quality of domestic airline [7] and the governor selection of the Asian Infrastructure Investment Bank [9]. 


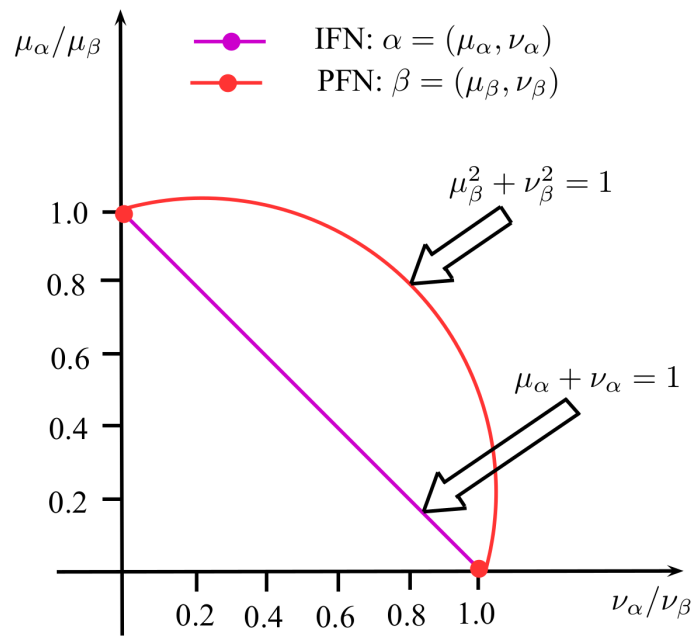

Figure 1. Comparison of spaces of the intuitionistic fuzzy numbers (IFNs) and the Pythagorean fuzzy numbers (PFNs).

A graph is a convenient way of interpreting information involving the relationship between objects. Fuzzy graphs are designed to represent structures of relationships between objects such that the existence of a concrete object (vertex) and relationship between two objects (edge) are matters of degree. The concept of fuzzy graphs was initiated by Kaufmann [10], based on Zadeh's fuzzy relations [11]. Later, another elaborated definition of fuzzy graph with fuzzy vertex and fuzzy edges was introduced by Rosenfeld [12] and obtaining analogs of several graph theoretical concepts such as paths, cycles and connectedness, etc., he developed the structure of fuzzy graphs. Mordeson and Peng [13] defined some operations on fuzzy graphs and investigated their properties. Later, the degrees of the vertices of the resultant graphs, obtained from two given fuzzy graphs using these operations, were determined in $[14,15]$. Parvathi and Karunambigai considered intuitionistic fuzzy graphs (IFGs). Later, IFGs were discussed by Akram and Davvaz [16]. After the inception of IFGs, many researchers $[17,18]$ generalized the concept of fuzzy graphs to IFGs. Naz et al. $[19,20]$ discussed some basic notions of single valued neutrosophic graphs along with its application in multi criteria decision-making. More recently, Akram et al. introduced many new concepts related to m-polar fuzzy graph, fuzzy soft graph, rough fuzzy graph, neutrosophic graph and their extensions [21-25]. This paper proposes a new graph, called Pythagorean fuzzy graph (PFG). In particular, we solve decision-making problems, including evaluation of hospitals, partner selection in supply chain management, electronic learning main factors evaluation by using PFGs.

\section{Pythagorean Fuzzy Graphs}

Definition 1. A PFS $\mathcal{Q}$ in $Z \times Z$ is said to be a Pythagorean fuzzy relation (PFR) in $Z$, denoted by

$$
\mathcal{Q}=\left\{\left\langle x y, \mu_{\mathcal{Q}}(x y), v_{\mathcal{Q}}(x y)\right\rangle \mid x y \in Z \times Z\right\},
$$

where $\mu_{\mathcal{Q}}: Z \times Z \rightarrow[0,1]$ and $v_{\mathcal{Q}}: Z \times Z \rightarrow[0,1]$ represent the membership and non-membership function of $\mathcal{Q}$, respectively, such that $0 \leq \mu_{\mathcal{Q}}^{2}(x y)+v_{\mathcal{Q}}^{2}(x y) \leq 1$ for all $x y \in Z \times Z$.

Definition 2. A PFG on a non-empty set $Z$ is a pair $\mathcal{G}=(\mathcal{P}, \mathcal{Q})$, where $\mathcal{P}$ is a PFS on $Z$ and $\mathcal{Q}$ is a PFR on Z such that

$$
\mu_{\mathcal{Q}}(x y) \leq \min \left\{\mu_{\mathcal{P}}(x), \mu_{\mathcal{P}}(y)\right\}, v_{\mathcal{Q}}(x y) \geq \max \left\{v_{\mathcal{P}}(x), v_{\mathcal{P}}(y)\right\}
$$

and $0 \leq \mu_{\mathcal{Q}}^{2}(x y)+v_{\mathcal{Q}}^{2}(x y) \leq 1$ for all $x, y \in Z$. 
Remark 1. - We call $\mathcal{P}$ and $\mathcal{Q}$ the Pythagorean fuzzy vertex set and the Pythagorean fuzzy edge set of $\mathcal{G}$, respectively.

- If $\mathcal{Q}$ is a symmetric on $\mathcal{P}$, then $\mathcal{G}=(\mathcal{P}, \mathcal{Q})$ is called PFG.

- If $\mathcal{Q}$ is not symmetric on $\mathcal{P}$, then $\mathcal{D}=(\mathcal{P}, \overrightarrow{\mathcal{Q}})$ is called Pythagorean fuzzy digraph.

- The proposed concept of PFGs is a generalization of the notion of Akram and Davoaz's IFGs [16].

Example 1. Consider a graph $G=(V, E)$, where $V=\{l, m, n, o, p, q, r\}$ and $E=\{l m, m n, n o, n r, p q, q r\}$. Let $\mathcal{P}$ and $\mathcal{Q}$ be the Pythagorean fuzzy vertex set and the Pythagorean fuzzy edge set defined on $V$ and $E$, respectively:

$$
\begin{aligned}
\mathcal{P} & =\left\langle\left(\frac{l}{0.7}, \frac{m}{0.6}, \frac{n}{0.7}, \frac{o}{0.8}, \frac{p}{0.4}, \frac{q}{0.5}, \frac{r}{0.6}\right),\left(\frac{l}{0.5}, \frac{m}{0.7}, \frac{n}{0.4}, \frac{o}{0.4}, \frac{p}{0.8}, \frac{q}{0.6}, \frac{r}{0.3}\right)\right\rangle, \\
\mathcal{Q} & =\left\langle\left(\frac{l m}{0.5}, \frac{m n}{0.4}, \frac{n o}{0.6}, \frac{n r}{0.3}, \frac{p q}{0.3}, \frac{q r}{0.4}\right),\left(\frac{l m}{0.8}, \frac{m n}{0.9}, \frac{n o}{0.5}, \frac{n r}{0.8}, \frac{p q}{0.9}, \frac{q r}{0.7}\right)\right\rangle .
\end{aligned}
$$

By direct calculations, it is easy to see from Figure 2 that $\mathcal{G}=(\mathcal{P}, \mathcal{Q})$ is a PFG.

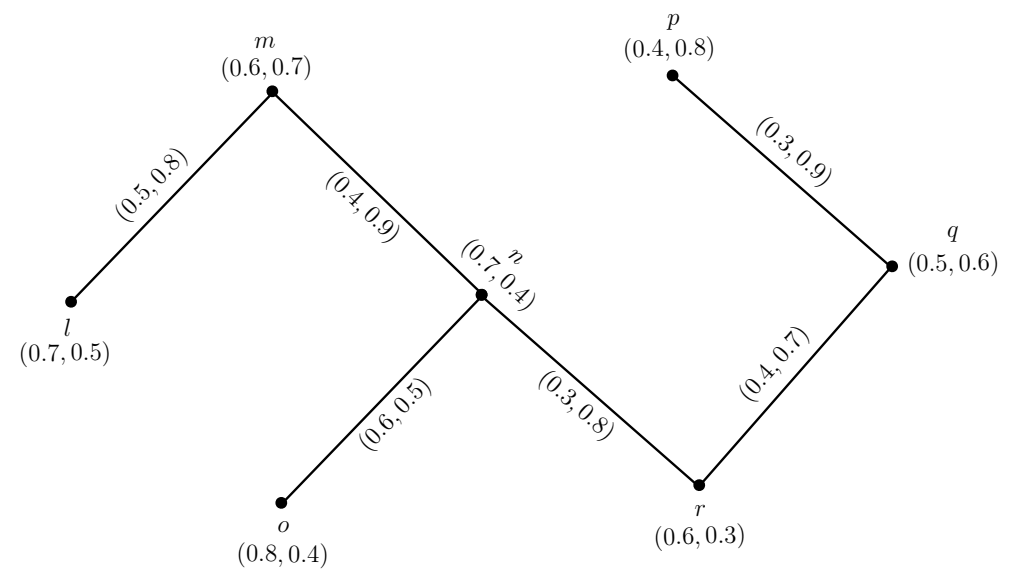

Figure 2. Pythagorean fuzzy graph (PFG).

Definition 3. The degree and total degree of a vertex $x \in V$ in a PFG $\mathcal{G}$ is defined as $\mathrm{d}_{\mathcal{G}}(x)=\left(\mathrm{d}_{\mu}(x), \mathrm{d}_{v}(x)\right)$ and $\operatorname{td}_{\mathcal{G}}(x)=\left(\operatorname{td}_{\mu}(x), \operatorname{td}_{v}(x)\right)$, respectively, where

$$
\begin{aligned}
\mathrm{d}_{\mu}(x) & =\sum_{x, y \neq x \in V} \mu_{\mathcal{Q}}(x y), \mathrm{d}_{v}(x)=\sum_{x, y \neq x \in V} v_{\mathcal{Q}}(x y), \\
\operatorname{td}_{\mu}(x) & =\sum_{x, y \neq x \in V} \mu_{\mathcal{Q}}(x y)+\mu_{\mathcal{P}}(x), \operatorname{td}_{v}(x)=\sum_{x, y \neq x \in V} v_{\mathcal{Q}}(x y)+v_{\mathcal{P}}(x) .
\end{aligned}
$$

The degree of a vertex $n$ is $\mathrm{d}_{\mathcal{G}}(n)=(1.3,2.2)$ and the total degree of a vertex $n$ in Figure 2 is $t \mathrm{~d}_{\mathcal{G}}(n)=(2.0,2.6)$.

Definition 4. Let $\mathcal{G}_{1}=\left(\mathcal{P}_{1}, \mathcal{Q}_{1}\right)$ and $\mathcal{G}_{2}=\left(\mathcal{P}_{2}, \mathcal{Q}_{2}\right)$ be two PFGs of the graphs $G_{1}=\left(V_{1}, E_{1}\right)$ and $G_{2}=\left(V_{2}, E_{2}\right)$, respectively. The direct product of $\mathcal{G}_{1}$ and $\mathcal{G}_{2}$ is denoted by $\mathcal{G}_{1} \times \mathcal{G}_{2}=\left(\mathcal{P}_{1} \times \mathcal{P}_{2}, \mathcal{Q}_{1} \times \mathcal{Q}_{2}\right)$ and defined as:

(i) $\left\{\begin{array}{l}\left(\mu_{\mathcal{P}_{1}} \times \mu_{\mathcal{P}_{2}}\right)\left(x_{1}, x_{2}\right)=\mu_{\mathcal{P}_{1}}\left(x_{1}\right) \wedge \mu_{\mathcal{P}_{2}}\left(x_{2}\right) \\ \left(v_{\mathcal{P}_{1}} \times v_{\mathcal{P}_{2}}\right)\left(x_{1}, x_{2}\right)=v_{\mathcal{P}_{1}}\left(x_{1}\right) \vee v_{\mathcal{P}_{2}}\left(x_{2}\right) \text { for all }\left(x_{1}, x_{2}\right) \in V_{1} \times V_{2}\end{array}\right.$

(ii) $\left\{\begin{array}{l}\left(\mu_{\mathcal{Q}_{1}} \times \mu_{\mathcal{Q}_{2}}\right)\left(\left(x_{1}, x_{2}\right)\left(y_{1}, y_{2}\right)\right)=\mu_{\mathcal{Q}_{1}}\left(x_{1} y_{1}\right) \wedge \mu_{\mathcal{Q}_{2}}\left(x_{2} y_{2}\right) \\ \left(v_{\mathcal{Q}_{1}} \times v_{\mathcal{Q}_{2}}\right)\left(\left(x_{1}, x_{2}\right)\left(y_{1}, y_{2}\right)\right)=v_{\mathcal{Q}_{1}}\left(x_{1} y_{1}\right) \vee v_{\mathcal{Q}_{2}}\left(x_{2} y_{2}\right) \text { for all } x_{1} y_{1} \in E_{1}, \text { for all } x_{2} y_{2} \in E_{2} .\end{array}\right.$

Proposition 1. Let $\mathcal{G}_{1}$ and $\mathcal{G}_{2}$ be the PFGs of the graphs $G_{1}$ and $G_{2}$, respectively. The direct product $\mathcal{G}_{1} \times \mathcal{G}_{2}$ of $\mathcal{G}_{1}$ and $\mathcal{G}_{2}$ is a PFG of $G_{1} \times G_{2}$. 
Definition 5. Let $\mathcal{G}_{1}=\left(\mathcal{P}_{1}, \mathcal{Q}_{1}\right)$ and $\mathcal{G}_{2}=\left(\mathcal{P}_{2}, \mathcal{Q}_{2}\right)$ be two PFGs. Then, for any vertex, $\left(x_{1}, x_{2}\right) \in V_{1} \times V_{2}$,

$$
\begin{aligned}
\left(d_{\mu}\right)_{\mathcal{G}_{1} \times \mathcal{G}_{2}}\left(x_{1}, x_{2}\right) & =\sum_{\left(x_{1}, x_{2}\right)\left(y_{1}, y_{2}\right) \in E_{1} \times E_{2}}\left(\mu_{\mathcal{Q}_{1}} \times \mu_{\mathcal{Q}_{2}}\right)\left(\left(x_{1}, x_{2}\right)\left(y_{1}, y_{2}\right)\right) \\
& =\sum_{x_{1} y_{1} \in E_{1}, x_{2} y_{2} \in E_{2}} \mu_{\mathcal{Q}_{1}}\left(x_{1} y_{1}\right) \wedge \mu_{\mathcal{Q}_{2}}\left(x_{2} y_{2}\right), \\
\left(d_{v}\right)_{\mathcal{G}_{1} \times \mathcal{G}_{2}}\left(x_{1}, x_{2}\right) & =\sum_{\left(x_{1}, x_{2}\right)\left(y_{1}, y_{2}\right) \in E_{1} \times E_{2}}\left(v_{\mathcal{Q}_{1}} \times v_{\mathcal{Q}_{2}}\right)\left(\left(x_{1}, x_{2}\right)\left(y_{1}, y_{2}\right)\right) \\
& =\sum_{x_{1} y_{1} \in E_{1}, x_{2} y_{2} \in E_{2}} v_{\mathcal{Q}_{1}}\left(x_{1} y_{1}\right) \vee v_{\mathcal{Q}_{2}}\left(x_{2} y_{2}\right) .
\end{aligned}
$$

Theorem 1. Let $\mathcal{G}_{1}=\left(\mathcal{P}_{1}, \mathcal{Q}_{1}\right)$ and $\mathcal{G}_{2}=\left(\mathcal{P}_{2}, \mathcal{Q}_{2}\right)$ be two PFGs. If $\mu_{\mathcal{Q}_{2}} \geq \mu_{\mathcal{Q}_{1}}, v_{\mathcal{Q}_{2}} \leq v_{\mathcal{Q}_{1}}$, then $d_{\mathcal{G}_{1} \times \mathcal{G}_{2}}\left(x_{1}, x_{2}\right)=d_{\mathcal{G}_{1}}\left(x_{1}\right)$ and if $\mu_{\mathcal{Q}_{1}} \geq \mu_{\mathcal{Q}_{2}}, v_{\mathcal{Q}_{1}} \leq v_{\mathcal{Q}_{2}}$, then $d_{\mathcal{G}_{1} \times \mathcal{G}_{2}}\left(x_{1}, x_{2}\right)=d_{\mathcal{G}_{2}}\left(x_{2}\right)$ for all $\left(x_{1}, x_{2}\right) \in V_{1} \times V_{2}$.

Proof. By definition of vertex degree of $\mathcal{G}_{1} \times \mathcal{G}_{2}$, we have

$$
\begin{aligned}
\left(d_{\mu}\right)_{\mathcal{G}_{1} \times \mathcal{G}_{2}}\left(x_{1}, x_{2}\right) & =\sum_{\left(x_{1}, x_{2}\right)\left(y_{1}, y_{2}\right) \in E_{1} \times E_{2}}\left(\mu_{\mathcal{Q}_{1}} \times \mu_{\mathcal{Q}_{2}}\right)\left(\left(x_{1}, x_{2}\right)\left(y_{1}, y_{2}\right)\right) \\
& =\sum_{x_{1} y_{1} \in E_{1}, x_{2} y_{2} \in E_{2}} \mu_{\mathcal{Q}_{1}}\left(x_{1} y_{1}\right) \wedge \mu_{\mathcal{Q}_{2}}\left(x_{2} y_{2}\right) \\
& =\sum_{x_{1} y_{1} \in E_{1}} \mu_{\mathcal{Q}_{1}}\left(x_{1} y_{1}\right) \quad\left(\text { since } \mu_{\mathcal{Q}_{2}} \geq \mu_{\mathcal{Q}_{1}}\right) \\
& =\left(d_{\mu}\right) \mathcal{G}_{1}\left(x_{1}\right), \\
\left(d_{v}\right)_{\mathcal{G}_{1} \times \mathcal{G}_{2}}\left(x_{1}, x_{2}\right) & =\sum_{\left(x_{1}, x_{2}\right)\left(y_{1}, y_{2}\right) \in E_{1} \times E_{2}}\left(v_{\mathcal{Q}_{1}} \times v_{\mathcal{Q}_{2}}\right)\left(\left(x_{1}, x_{2}\right)\left(y_{1}, y_{2}\right)\right) \\
& =\sum_{x_{1} y_{1} \in E_{1}, x_{2} y_{2} \in E_{2}} v_{\mathcal{Q}_{1}}\left(x_{1} y_{1}\right) \vee v_{\mathcal{Q}_{2}}\left(x_{2} y_{2}\right) \\
& =\sum_{x_{1} y_{1} \in E_{1}} v_{\mathcal{Q}_{1}}\left(x_{1} y_{1}\right) \quad\left(\text { since } v_{\mathcal{Q}_{2}} \leq v_{\mathcal{Q}_{1}}\right) \\
& =\left(d_{v}\right)_{\mathcal{G}_{1}}\left(x_{1}\right) .
\end{aligned}
$$

Hence, $d_{\mathcal{G}_{1} \times \mathcal{G}_{2}}\left(x_{1}, x_{2}\right)=d_{\mathcal{G}_{1}}\left(x_{1}\right)$. Similarly, it is easy to show that, if $\mu_{\mathcal{Q}_{1}} \geq \mu_{\mathcal{Q}_{2}}, v_{\mathcal{Q}_{1}} \leq v_{\mathcal{Q}_{2}}$, then $d_{\mathcal{G}_{1} \times \mathcal{G}_{2}}\left(x_{1}, x_{2}\right)=d_{\mathcal{G}_{2}}\left(x_{2}\right)$.

Definition 6. Let $\mathcal{G}_{1}=\left(\mathcal{P}_{1}, \mathcal{Q}_{1}\right)$ and $\mathcal{G}_{2}=\left(\mathcal{P}_{2}, \mathcal{Q}_{2}\right)$ be two PFGs. For any vertex $\left(x_{1}, x_{2}\right) \in V_{1} \times V_{2}$,

$$
\begin{aligned}
\left(t d_{\mu}\right)_{\mathcal{G}_{1} \times \mathcal{G}_{2}}\left(x_{1}, x_{2}\right) & =\sum_{\left(x_{1}, x_{2}\right)\left(y_{1}, y_{2}\right) \in E_{1} \times E_{2}}\left(\mu_{\mathcal{Q}_{1}} \times \mu_{\mathcal{Q}_{2}}\right)\left(\left(x_{1}, x_{2}\right)\left(y_{1}, y_{2}\right)\right)+\left(\mu_{\mathcal{P}_{1}} \times \mu_{\mathcal{P}_{2}}\right)\left(x_{1}, x_{2}\right) \\
& =\sum_{x_{1} y_{1} \in E_{1}, x_{2} y_{2} \in E_{2}} \mu_{\mathcal{Q}_{1}}\left(x_{1} y_{1}\right) \wedge \mu_{\mathcal{Q}_{2}}\left(x_{2} y_{2}\right)+\mu_{\mathcal{P}_{1}}\left(x_{1}\right) \wedge \mu_{\mathcal{P}_{2}}\left(x_{2}\right), \\
\left(t d_{v}\right)_{\mathcal{G}_{1} \times \mathcal{G}_{2}}\left(x_{1}, x_{2}\right) & =\sum_{\left(x_{1}, x_{2}\right)\left(y_{1}, y_{2}\right) \in E_{1} \times E_{2}}\left(v_{\mathcal{Q}_{1}} \times v_{\mathcal{Q}_{2}}\right)\left(\left(x_{1}, x_{2}\right)\left(y_{1}, y_{2}\right)\right)+\left(v_{\mathcal{P}_{1}} \times v_{\mathcal{P}_{2}}\right)\left(x_{1}, x_{2}\right) \\
& =\sum_{x_{1} y_{1} \in E_{1}, x_{2} y_{2} \in E_{2}} v_{\mathcal{Q}_{1}}\left(x_{1} y_{1}\right) \vee v_{\mathcal{Q}_{2}}\left(x_{2} y_{2}\right)+v_{\mathcal{P}_{1}}\left(x_{1}\right) \vee v_{\mathcal{P}_{2}}\left(x_{2}\right) .
\end{aligned}
$$

Theorem 2. Let $\mathcal{G}_{1}=\left(\mathcal{P}_{1}, \mathcal{Q}_{1}\right)$ and $\mathcal{G}_{2}=\left(\mathcal{P}_{2}, \mathcal{Q}_{2}\right)$ be two PFGs. If

(i) $\mu_{\mathcal{Q}_{2}} \geq \mu_{\mathcal{Q}_{1}}$, then $\left(t d_{\mu}\right)_{\mathcal{G}_{1} \times \mathcal{G}_{2}}\left(x_{1}, x_{2}\right)=\left(d_{\mu}\right)_{\mathcal{G}_{1}}\left(x_{1}\right)+\mu_{\mathcal{P}_{1}}\left(x_{1}\right) \wedge \mu_{\mathcal{P}_{2}}\left(x_{2}\right)$; 
(ii) $v_{\mathcal{Q}_{2}} \leq v_{\mathcal{Q}_{1}}$, then $\left(t d_{v}\right)_{\mathcal{G}_{1} \times \mathcal{G}_{2}}\left(x_{1}, x_{2}\right)=\left(d_{v}\right)_{\mathcal{G}_{1}}\left(x_{1}\right)+v_{\mathcal{P}_{1}}\left(x_{1}\right) \vee v_{\mathcal{P}_{2}}\left(x_{2}\right)$;

(iii) $\mu_{\mathcal{Q}_{1}} \geq \mu_{\mathcal{Q}_{2}}$, then $\left(t d_{\mu}\right)_{\mathcal{G}_{1} \times \mathcal{G}_{2}}\left(x_{1}, x_{2}\right)=\left(d_{\mu}\right)_{\mathcal{G}_{2}}\left(x_{2}\right)+\mu_{\mathcal{P}_{1}}\left(x_{1}\right) \wedge \mu_{\mathcal{P}_{2}}\left(x_{2}\right)$;

(vi) $v_{\mathcal{Q}_{1}} \leq v_{\mathcal{Q}_{2}}$, then $\left(t d_{v}\right)_{\mathcal{G}_{1} \times \mathcal{G}_{2}}\left(x_{1}, x_{2}\right)=\left(d_{v}\right)_{\mathcal{G}_{2}}\left(x_{2}\right)+v_{\mathcal{P}_{1}}\left(x_{1}\right) \vee v_{\mathcal{P}_{2}}\left(x_{2}\right)$

for all $\left(x_{1}, x_{2}\right) \in V_{1} \times V_{2}$.

Proof. The proof is straightforward using Definition 6 and Theorem 1.

Example 2. Consider two PFGs $\mathcal{G}_{1}=\left(\mathcal{P}_{1}, \mathcal{Q}_{1}\right)$ and $\mathcal{G}_{2}=\left(\mathcal{P}_{2}, \mathcal{Q}_{2}\right)$ on $V_{1}=\{l, m, n\}$ and $V_{2}=\{o, p\}$, respectively, as shown in Figure 3. Their direct product $\mathcal{G}_{1} \times \mathcal{G}_{2}$ is shown in Figure 4.

$\mathcal{G}_{1}$

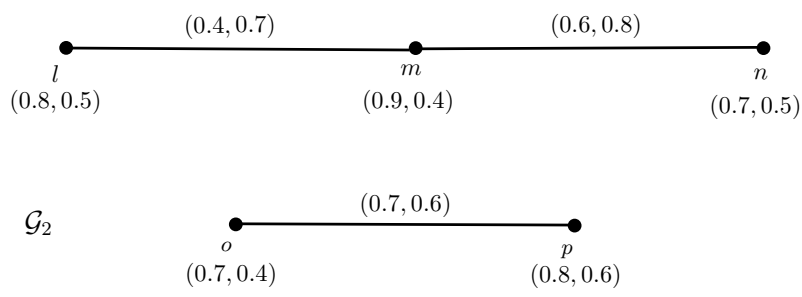

Figure 3. PFGs.

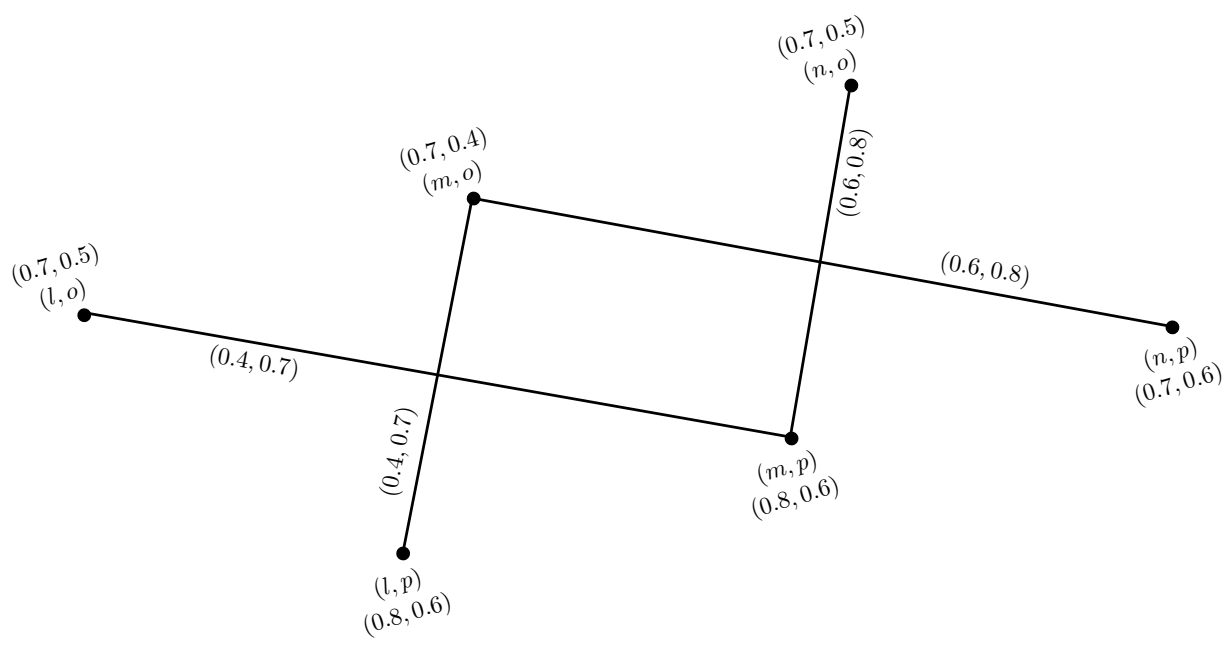

Figure 4. Direct product of two PFGs.

Since $\mu_{\mathcal{Q}_{2}} \geq \mu_{\mathcal{Q}_{1}}, v_{\mathcal{Q}_{2}} \leq v_{\mathcal{Q}_{1}}$, so, by Theorem 1, we have

$$
\left(d_{\mu}\right)_{\mathcal{G}_{1} \times \mathcal{G}_{2}}(m, p)=\left(d_{\mu}\right)_{\mathcal{G}_{1}}(m)=1.0,\left(d_{v}\right)_{\mathcal{G}_{1} \times \mathcal{G}_{2}}(m, p)=\left(d_{v}\right)_{\mathcal{G}_{1}}(m)=1.5
$$

Therefore, $d_{\mathcal{G}_{1} \times \mathcal{G}_{2}}(m, p)=(1.0,1.5)$.

In addition, by Theorem 2, we have

$$
\begin{aligned}
& \left(t d_{\mu}\right)_{\mathcal{G}_{1} \times \mathcal{G}_{2}}(m, p)=\left(d_{\mu}\right)_{\mathcal{G}_{1}}(m)+\mu_{\mathcal{P}_{1}}(m) \wedge \mu_{\mathcal{P}_{2}}(p)=1.8 \\
& \left(t d_{v}\right)_{\mathcal{G}_{1} \times \mathcal{G}_{2}}(m, p)=\left(d_{v}\right)_{\mathcal{G}_{1}}(m)+v_{\mathcal{P}_{1}}(m) \vee v_{\mathcal{P}_{2}}(p)=2.1
\end{aligned}
$$

Therefore, $t d_{\mathcal{G}_{1} \times \mathcal{G}_{2}}(m, p)=(1.8,2.1)$.

Similarly, it is easy to find the degree and total degree of all the vertices in $\mathcal{G}_{1} \times \mathcal{G}_{2}$.

Definition 7. Let $\mathcal{G}_{1}=\left(\mathcal{P}_{1}, \mathcal{Q}_{1}\right)$ and $\mathcal{G}_{2}=\left(\mathcal{P}_{2}, \mathcal{Q}_{2}\right)$ be two PFGs of $G_{1}=\left(V_{1}, E_{1}\right)$ and $G_{2}=\left(V_{2}, E_{2}\right)$, respectively. The Cartesian product of $\mathcal{G}_{1}$ and $\mathcal{G}_{2}$ is denoted by $\mathcal{G}_{1} \square \mathcal{G}_{2}=\left(\mathcal{P}_{1} \square \mathcal{P}_{2}, \mathcal{Q}_{1} \square \mathcal{Q}_{2}\right)$ and defined as: 

(i) $\left\{\begin{array}{l}\left(\mu_{\mathcal{P}_{1}} \square \mu_{\mathcal{P}_{2}}\right)\left(x_{1}, x_{2}\right)=\mu_{\mathcal{P}_{1}}\left(x_{1}\right) \wedge \mu_{\mathcal{P}_{2}}\left(x_{2}\right) \\ \left(v_{\mathcal{P}_{1}} \square v_{\mathcal{P}_{2}}\right)\left(x_{1}, x_{2}\right)=v_{\mathcal{P}_{1}}\left(x_{1}\right) \vee v_{\mathcal{P}_{2}}\left(x_{2}\right) \quad \text { for all }\left(x_{1}, x_{2}\right) \in V_{1} \times V_{2},\end{array}\right.$
(ii) $\left\{\begin{array}{l}\left(\mu_{\mathcal{Q}_{1}} \square \mu_{\mathcal{Q}_{2}}\right)\left(\left(x, x_{2}\right)\left(x, y_{2}\right)\right)=\mu_{\mathcal{P}_{1}}(x) \wedge \mu_{\mathcal{Q}_{2}}\left(x_{2} y_{2}\right) \\ \left(v_{\mathcal{Q}_{1}} \square v_{\mathcal{Q}_{2}}\right)\left(\left(x, x_{2}\right)\left(x, y_{2}\right)\right)=v_{\mathcal{P}_{1}}(x) \vee v_{\mathcal{Q}_{2}}\left(x_{2} y_{2}\right) \quad \text { for all } x \in V_{1} \text {, for all } x_{2} y_{2} \in E_{2} \text {, }\end{array}\right.$
(iii) $\left\{\begin{array}{l}\left(\mu_{\mathcal{Q}_{1}} \square \mu_{\mathcal{Q}_{2}}\right)\left(\left(x_{1}, z\right)\left(y_{1}, z\right)\right)=\mu_{\mathcal{Q}_{1}}\left(x_{1} y_{1}\right) \wedge \mu_{\mathcal{P}_{2}}(z) \\ \left(v_{\mathcal{Q}_{1}} \square v_{\mathcal{Q}_{2}}\right)\left(\left(x_{1}, z\right)\left(y_{1}, z\right)\right)=v_{\mathcal{Q}_{1}}\left(x_{1} y_{1}\right) \vee v_{\mathcal{P}_{2}}(z) \quad \text { for all } z \in V_{2}, \text { for all } x_{1} y_{1} \in E_{1} .\end{array}\right.$

Proposition 2. Let $\mathcal{G}_{1}$ and $\mathcal{G}_{2}$ be the PFGs of the graphs $G_{1}$ and $G_{2}$, respectively. The Cartesian product $\mathcal{G}_{1} \square \mathcal{G}_{2}$ of $\mathcal{G}_{1}$ and $\mathcal{G}_{2}$ is a PFG of $G_{1} \square G_{2}$.

Definition 8. Let $\mathcal{G}_{1}=\left(\mathcal{P}_{1}, \mathcal{Q}_{1}\right)$ and $\mathcal{G}_{2}=\left(\mathcal{P}_{2}, \mathcal{Q}_{2}\right)$ be two PFGs. For any vertex $\left(x_{1}, x_{2}\right) \in V_{1} \times V_{2}$,

$$
\begin{aligned}
\left(d_{\mu}\right)_{\mathcal{G}_{1} \square \mathcal{G}_{2}}\left(x_{1}, x_{2}\right) & =\sum_{\left(x_{1}, x_{2}\right)\left(y_{1}, y_{2}\right) \in E_{1} \square E_{2}}\left(\mu_{\mathcal{Q}_{1}} \square \mu_{\mathcal{Q}_{2}}\right)\left(\left(x_{1}, x_{2}\right)\left(y_{1}, y_{2}\right)\right) \\
& =\sum_{x_{1}=y_{1}, x_{2} y_{2} \in E_{2}} \mu_{\mathcal{P}_{1}}\left(x_{1}\right) \wedge \mu_{\mathcal{Q}_{2}}\left(x_{2} y_{2}\right)+\sum_{x_{2}=y_{2}, x_{1} y_{1} \in E_{1}} \mu_{\mathcal{P}_{2}}\left(x_{2}\right) \wedge \mu_{\mathcal{Q}_{1}}\left(x_{1} y_{1}\right), \\
\left(d_{v}\right)_{\mathcal{G}_{1} \square \mathcal{G}_{2}}\left(x_{1}, x_{2}\right) & =\sum_{\left(x_{1}, x_{2}\right)\left(y_{1}, y_{2}\right) \in E_{1} \square E_{2}}\left(v_{\mathcal{Q}_{1}} \square v_{\mathcal{Q}_{2}}\right)\left(\left(x_{1}, x_{2}\right)\left(y_{1}, y_{2}\right)\right) \\
& =\sum_{x_{1}=y_{1}, x_{2} y_{2 \in E_{2}}} v_{\mathcal{P}_{1}}\left(x_{1}\right) \vee v_{\mathcal{Q}_{2}}\left(x_{2} y_{2}\right)+\sum_{x_{2}=y_{2}, x_{1} y_{1} \in E_{1}} v_{\mathcal{P}_{2}}\left(x_{2}\right) \vee v_{\mathcal{Q}_{1}}\left(x_{1} y_{1}\right) .
\end{aligned}
$$

Theorem 3. Let $\mathcal{G}_{1}=\left(\mathcal{P}_{1}, \mathcal{Q}_{1}\right)$ and $\mathcal{G}_{2}=\left(\mathcal{P}_{2}, \mathcal{Q}_{2}\right)$ be two PFGs. If $\mu_{\mathcal{P}_{1}} \geq \mu_{\mathcal{Q}_{2}}, v_{\mathcal{P}_{1}} \leq v_{\mathcal{Q}_{2}}$ and $\mu_{\mathcal{P}_{2}} \geq \mu_{\mathcal{Q}_{1}}, v_{\mathcal{P}_{2}} \leq v_{\mathcal{Q}_{1}}$. Then $d_{\mathcal{G}_{1} \square \mathcal{G}_{2}}\left(x_{1}, x_{2}\right)=d_{\mathcal{G}_{1}}\left(x_{1}\right)+d_{\mathcal{G}_{2}}\left(x_{2}\right)$ for all $\left(x_{1}, x_{2}\right) \in V_{1} \times V_{2}$.

Proof. By definition of vertex degree of $\mathcal{G}_{1} \square \mathcal{G}_{2}$, we have

$$
\begin{aligned}
\left(d_{\mu}\right)_{\mathcal{G}_{1} \square \mathcal{G}_{2}}\left(x_{1}, x_{2}\right)= & \sum_{\left(x_{1}, x_{2}\right)\left(y_{1}, y_{2}\right) \in E_{1} \square E_{2}}\left(\mu_{\mathcal{Q}_{1}} \square \mu_{\mathcal{Q}_{2}}\right)\left(\left(x_{1}, x_{2}\right)\left(y_{1}, y_{2}\right)\right) \\
= & \sum_{x_{1}=y_{1}, x_{2} y_{2} \in E_{2}} \mu_{\mathcal{P}_{1}}\left(x_{1}\right) \wedge \mu_{\mathcal{Q}_{2}}\left(x_{2} y_{2}\right)+\sum_{x_{2}=y_{2}, x_{1} y_{1} \in E_{1}} \mu_{\mathcal{P}_{2}}\left(x_{2}\right) \wedge \mu_{\mathcal{Q}_{1}}\left(x_{1} y_{1}\right) \\
= & \sum_{x_{2} y_{2 \in E_{2}}} \mu_{\mathcal{Q}_{2}}\left(x_{2} y_{2}\right)+\sum_{x_{1} y_{1} \in E_{1}} \mu_{\mathcal{Q}_{1}}\left(x_{1} y_{1}\right) \\
& \left(\text { by using } \mu_{\mathcal{P}_{1}} \geq \mu_{\mathcal{Q}_{2}} \text { and } \mu_{\mathcal{P}_{2}} \geq \mu_{\mathcal{Q}_{1}}\right) \\
= & \left(d_{\mu}\right)_{\mathcal{G}_{1}}\left(x_{1}\right)+\left(d_{\mu}\right) \mathcal{G}_{2}\left(x_{2}\right), \\
\left(d_{v}\right)_{\mathcal{G}_{1} \square \mathcal{G}_{2}}\left(x_{1}, x_{2}\right)= & \sum_{\left(x_{1}, x_{2}\right)\left(y_{1}, y_{2}\right) \in E_{1} \square E_{2}}\left(v_{\mathcal{Q}_{1}} \square v_{\mathcal{Q}_{2}}\right)\left(\left(x_{1}, x_{2}\right)\left(y_{1}, y_{2}\right)\right) \\
= & \sum_{x_{1}=y_{1}, x_{2} y_{2 \in E_{2}}} v_{\mathcal{P}_{1}}\left(x_{1}\right) \vee v_{\mathcal{Q}_{2}}\left(x_{2} y_{2}\right)+\sum_{x_{2}=y_{2}, x_{1} y_{1} \in E_{1}} v_{\mathcal{P}_{2}}\left(x_{2}\right) \vee v_{\mathcal{Q}_{1}}\left(x_{1} y_{1}\right) \\
= & \sum_{x_{2} y_{2} \in E_{2}} v_{\mathcal{Q}_{2}}\left(x_{2} y_{2}\right)+\sum_{x_{1} y_{1} \in E_{1}} v_{\mathcal{Q}_{1}}\left(x_{1} y_{1}\right) \\
& \left(\text { by using } v_{\mathcal{P}_{1}} \leq v_{\mathcal{Q}_{2}} \text { and } v_{\mathcal{P}_{2}} \leq v_{\mathcal{Q}_{1}}\right) \\
= & \left(d_{v}\right)_{\mathcal{G}_{1}}\left(x_{1}\right)+\left(d_{v}\right) \\
&
\end{aligned}
$$

Hence, $d_{\mathcal{G}_{1} \square \mathcal{G}_{2}}\left(x_{1}, x_{2}\right)=d_{\mathcal{G}_{1}}\left(x_{1}\right)+d_{\mathcal{G}_{2}}\left(x_{2}\right)$. 
Definition 9. Let $\mathcal{G}_{1}=\left(\mathcal{P}_{1}, \mathcal{Q}_{1}\right)$ and $\mathcal{G}_{2}=\left(\mathcal{P}_{2}, \mathcal{Q}_{2}\right)$ be two PFGs. For any vertex $\left(x_{1}, x_{2}\right) \in V_{1} \times V_{2}$,

$$
\begin{aligned}
\left(t d_{\mu}\right)_{\mathcal{G}_{1} \square \mathcal{G}_{2}}\left(x_{1}, x_{2}\right)= & \sum_{\left(x_{1}, x_{2}\right)\left(y_{1}, y_{2}\right) \in E_{1} \square E_{2}}\left(\mu_{\mathcal{Q}_{1}} \square \mu_{\mathcal{Q}_{2}}\right)\left(\left(x_{1}, x_{2}\right)\left(y_{1}, y_{2}\right)\right)+\left(\mu_{\mathcal{P}_{1}} \square \mu_{\mathcal{P}_{2}}\right)\left(x_{1}, x_{2}\right) \\
= & \sum_{x_{1}=y_{1}, x_{2} y_{2} \in E_{2}} \mu_{\mathcal{P}_{1}}\left(x_{1}\right) \wedge \mu_{\mathcal{Q}_{2}}\left(x_{2} y_{2}\right)+\sum_{x_{2}=y_{2}, x_{1} y_{1} \in E_{1}} \mu_{\mathcal{P}_{2}}\left(x_{2}\right) \wedge \mu_{\mathcal{Q}_{1}}\left(x_{1} y_{1}\right) \\
& +\mu_{\mathcal{P}_{1}}\left(x_{1}\right) \wedge \mu_{\mathcal{P}_{2}}\left(x_{2}\right), \\
\left(t d_{v}\right)_{\mathcal{G}_{1} \square \mathcal{G}_{2}}\left(x_{1}, x_{2}\right)= & \sum_{\left(x_{1}, x_{2}\right)\left(y_{1}, y_{2}\right) \in E_{1} \square E_{2}}\left(v_{\mathcal{Q}_{1}} \square v_{\mathcal{Q}_{2}}\right)\left(\left(x_{1}, x_{2}\right)\left(y_{1}, y_{2}\right)\right)+\left(v_{\mathcal{P}_{1}} \square v_{\mathcal{P}_{2}}\right)\left(x_{1}, x_{2}\right) \\
= & \sum_{x_{1}=y_{1}, x_{2} y_{2 \in E_{2}}} v_{\mathcal{P}_{1}}\left(x_{1}\right) \vee v_{\mathcal{Q}_{2}}\left(x_{2} y_{2}\right)+\sum_{x_{2}=y_{2}, x_{1} y_{1} \in E_{1}} v_{\mathcal{P}_{2}}\left(x_{2}\right) \vee v_{\mathcal{Q}_{1}}\left(x_{1} y_{1}\right) \\
& +v_{\mathcal{P}_{1}}\left(x_{1}\right) \vee v_{\mathcal{P}_{2}}\left(x_{2}\right) .
\end{aligned}
$$

Theorem 4. Let $\mathcal{G}_{1}=\left(\mathcal{P}_{1}, \mathcal{Q}_{1}\right)$ and $\mathcal{G}_{2}=\left(\mathcal{P}_{2}, \mathcal{Q}_{2}\right)$ be two PFGs. If

(i) $\mu_{\mathcal{P}_{1}} \geq \mu_{\mathcal{Q}_{2}}$ and $\mu_{\mathcal{P}_{2}} \geq \mu_{\mathcal{Q}_{1}}$, then $\left(t d_{\mu}\right)_{\mathcal{G}_{1} \square \mathcal{G}_{2}}\left(x_{1}, x_{2}\right)=\left(t d_{\mu}\right)_{\mathcal{G}_{1}}\left(x_{1}\right)+\left(t d_{\mu}\right)_{\mathcal{G}_{2}}\left(x_{2}\right)-\mu_{\mathcal{P}_{1}}\left(x_{1}\right) \vee$ $\mu_{\mathcal{P}_{2}}\left(x_{2}\right)$

(ii) $v_{\mathcal{P}_{1}} \leq v_{\mathcal{Q}_{2}}$ and $v_{\mathcal{P}_{2}} \leq v_{\mathcal{Q}_{1}}$, then $\left(t d_{v}\right)_{\mathcal{G}_{1} \square \mathcal{G}_{2}}\left(x_{1}, x_{2}\right)=\left(t d_{v}\right)_{\mathcal{G}_{1}}\left(x_{1}\right)+\left(t d_{v}\right)_{\mathcal{G}_{2}}\left(x_{2}\right)$

$-v_{\mathcal{P}_{1}}\left(x_{1}\right) \wedge v_{\mathcal{P}_{2}}\left(x_{2}\right)$

for all $\left(x_{1}, x_{2}\right) \in V_{1} \times V_{2}$.

Proof. By definition of vertex total degree of $\mathcal{G}_{1} \square \mathcal{G}_{2}$,

(i) If $\mu_{\mathcal{P}_{1}} \geq \mu_{\mathcal{Q}_{2}}, \mu_{\mathcal{P}_{2}} \geq \mu_{\mathcal{Q}_{1}}$

$$
\begin{aligned}
\left(t d_{\mu}\right)_{\mathcal{G}_{1} \square \mathcal{G}_{2}}\left(x_{1}, x_{2}\right)= & \sum_{x_{1}=y_{1}, x_{2} y_{2} \in E_{2}} \mu_{\mathcal{P}_{1}}\left(x_{1}\right) \wedge \mu_{\mathcal{Q}_{2}}\left(x_{2} y_{2}\right)+\sum_{x_{2}=y_{2}, x_{1} y_{1} \in E_{1}} \mu_{\mathcal{P}_{2}}\left(x_{2}\right) \wedge \mu_{\mathcal{Q}_{1}}\left(x_{1} y_{1}\right) \\
& +\mu_{\mathcal{P}_{1}}\left(x_{1}\right) \wedge \mu_{\mathcal{P}_{2}}\left(x_{2}\right) \\
= & \sum_{x_{2} y_{2 \in E_{2}}} \mu_{\mathcal{Q}_{2}}\left(x_{2} y_{2}\right)+\sum_{x_{1} y_{1} \in E_{1}} \mu_{\mathcal{Q}_{1}}\left(x_{1} y_{1}\right)+\mu_{\mathcal{P}_{1}}\left(x_{1}\right)+\mu_{\mathcal{P}_{2}}\left(x_{2}\right) \\
= & -\mu_{\mathcal{P}_{1}}\left(x_{1}\right) \vee \mu_{\mathcal{P}_{2}}\left(x_{2}\right) \\
& \sum_{2 y_{2} \in E_{2}} \mu_{\mathcal{Q}_{2}}\left(x_{2} y_{2}\right)+\mu_{\mathcal{P}_{2}}\left(x_{2}\right)+\sum_{x_{1} y_{1} \in E_{1}} \mu_{\mathcal{Q}_{1}}\left(x_{1} y_{1}\right)+\mu_{\mathcal{P}_{1}}\left(x_{1}\right) \\
& -\mu_{\mathcal{P}_{1}}\left(x_{1}\right) \vee \mu_{\mathcal{P}_{2}}\left(x_{2}\right) \\
= & \left(t d_{\mu}\right)_{\mathcal{G}_{1}}\left(x_{1}\right)+\left(t d_{\mu}\right)_{\mathcal{G}_{2}}\left(x_{2}\right)-\mu_{\mathcal{P}_{1}}\left(x_{1}\right) \vee \mu_{\mathcal{P}_{2}}\left(x_{2}\right)
\end{aligned}
$$

(ii) If $v_{\mathcal{P}_{1}} \leq v_{\mathcal{Q}_{2}}, v_{\mathcal{P}_{2}} \leq v_{\mathcal{Q}_{1}}$

$$
\begin{aligned}
\left(t d_{v}\right)_{\mathcal{G}_{1} \square \mathcal{G}_{2}}\left(x_{1}, x_{2}\right)= & \sum_{x_{1}=y_{1}, x_{2} y_{2 \in E_{2}}} v_{\mathcal{P}_{1}}\left(x_{1}\right) \vee v_{\mathcal{Q}_{2}}\left(x_{2} y_{2}\right)+\sum_{x_{2}=y_{2}, x_{1} y_{1} \in E_{1}} v_{\mathcal{P}_{2}}\left(x_{2}\right) \vee v_{\mathcal{Q}_{1}}\left(x_{1} y_{1}\right) \\
& +v_{\mathcal{P}_{1}}\left(x_{1}\right) \vee v_{\mathcal{P}_{2}}\left(x_{2}\right) \\
= & \sum_{x_{2} y_{2} \in E_{2}} v_{\mathcal{Q}_{2}}\left(x_{2} y_{2}\right)+\sum_{x_{1} y_{1} \in E_{1}} v_{\mathcal{Q}_{1}}\left(x_{1} y_{1}\right)+v_{\mathcal{P}_{1}}\left(x_{1}\right)+v_{\mathcal{P}_{2}}\left(x_{2}\right) \\
& -v_{\mathcal{P}_{1}}\left(x_{1}\right) \wedge v_{\mathcal{P}_{2}}\left(x_{2}\right) \\
= & \left(t d_{v}\right)_{\mathcal{G}_{1}}\left(x_{1}\right)+\left(t d_{v}\right)_{\mathcal{G}_{2}}\left(x_{2}\right)-v_{\mathcal{P}_{1}}\left(x_{1}\right) \wedge v_{\mathcal{P}_{2}}\left(x_{2}\right) .
\end{aligned}
$$

Example 3. Consider two PFGs $\mathcal{G}_{1}$ and $\mathcal{G}_{2}$ as in Example 2, where $\mu_{\mathcal{P}_{1}} \geq \mu_{\mathcal{Q}_{2}}, v_{\mathcal{P}_{1}} \leq v_{\mathcal{Q}_{2}}$ and $\mu_{\mathcal{P}_{2}} \geq \mu_{\mathcal{Q}_{1}}, v_{\mathcal{P}_{2}} \leq v_{\mathcal{Q}_{1}}$. Their Cartesian product $\mathcal{G}_{1} \square \mathcal{G}_{2}$ is shown in Figure 5 . 


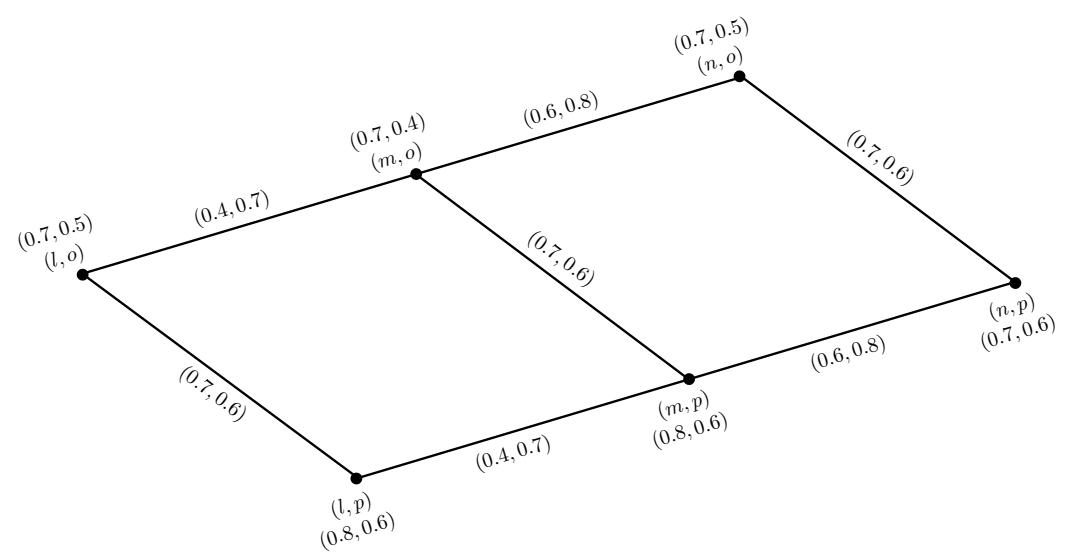

Figure 5. Cartesian product of two PFGs.

Then, by Theorem 3, we have

$$
\begin{gathered}
\left(d_{\mu}\right)_{\mathcal{G}_{1} \square \mathcal{G}_{2}}(l, o)=\left(d_{\mu}\right)_{\mathcal{G}_{1}}(l)+\left(d_{\mu}\right)_{\mathcal{G}_{2}}(o)=1.1, \\
\left(d_{v}\right)_{\mathcal{G}_{1} \square \mathcal{G}_{2}}(l, o)=\left(d_{\nu}\right)_{\mathcal{G}_{1}}(l)+\left(d_{v}\right)_{\mathcal{G}_{2}}(o)=1.3 .
\end{gathered}
$$

Therefore, $d_{\mathcal{G}_{1}} \square \mathcal{G}_{2}(l, o)=(1.1,1.3)$.

In addition, by Theorem 4, we have

$$
\begin{aligned}
& \left(t d_{\mu}\right)_{\mathcal{G}_{1} \square \mathcal{G}_{2}}(l, o)=\left(t d_{\mu}\right)_{\mathcal{G}_{1}}(l)+\left(t d_{\mu}\right)_{\mathcal{G}_{2}}(o)-\mu_{\mathcal{P}_{1}}(l) \vee \mu_{\mathcal{P}_{2}}(o)=1.8, \\
& \left(t d_{v}\right)_{\mathcal{G}_{1} \square \mathcal{G}_{2}}(l, o)=\left(t d_{v}\right)_{\mathcal{G}_{1}}(l)+\left(t d_{v}\right)_{\mathcal{G}_{2}}(o)-v_{\mathcal{P}_{1}}(l) \wedge v_{\mathcal{P}_{2}}(o)=1.8 .
\end{aligned}
$$

Therefore, $t d_{\mathcal{G}_{1}} \square \mathcal{G}_{2}(l, o)=(1.8,1.8)$.

Similarly, we can find the degree and total degree of all the vertices in $\mathcal{G}_{1} \square \mathcal{G}_{2}$.

Definition 10. Let $\mathcal{G}_{1}=\left(\mathcal{P}_{1}, \mathcal{Q}_{1}\right)$ and $\mathcal{G}_{2}=\left(\mathcal{P}_{2}, \mathcal{Q}_{2}\right)$ be two PFGs of the graphs $G_{1}=\left(V_{1}, E_{1}\right)$ and $G_{2}=\left(V_{2}, E_{2}\right)$, respectively. The semi-strong product of $\mathcal{G}_{1}$ and $\mathcal{G}_{2}$, denoted by $\mathcal{G}_{1} \bullet \mathcal{G}_{2}=\left(\mathcal{P}_{1} \bullet \mathcal{P}_{2}, \mathcal{Q}_{1} \bullet \mathcal{Q}_{2}\right)$, is defined as:

(i) $\left\{\begin{array}{l}\left(\mu_{\mathcal{P}_{1}} \bullet \mu_{\mathcal{P}_{2}}\right)\left(x_{1}, x_{2}\right)=\mu_{\mathcal{P}_{1}}\left(x_{1}\right) \wedge \mu_{\mathcal{P}_{2}}\left(x_{2}\right) \\ \left(v_{\mathcal{P}_{1}} \bullet v_{\mathcal{P}_{2}}\right)\left(x_{1}, x_{2}\right)=v_{\mathcal{P}_{1}}\left(x_{1}\right) \vee v_{\mathcal{P}_{2}}\left(x_{2}\right)\end{array} \quad\right.$ for all $\left(x_{1}, x_{2}\right) \in V_{1} \times V_{2}$,

(ii) $\left\{\begin{array}{l}\left(\mu_{\mathcal{Q}_{1}} \bullet \mu_{\mathcal{Q}_{2}}\right)\left(\left(x, x_{2}\right)\left(x, y_{2}\right)\right)=\mu_{\mathcal{P}_{1}}(x) \wedge \mu_{\mathcal{Q}_{2}}\left(x_{2} y_{2}\right) \\ \left(v_{\mathcal{Q}_{1}} \bullet v_{\mathcal{Q}_{2}}\right)\left(\left(x, x_{2}\right)\left(x, y_{2}\right)\right)=v_{\mathcal{P}_{1}}(x) \vee v_{\mathcal{Q}_{2}}\left(x_{2} y_{2}\right)\end{array}\right.$ for all $x \in V_{1}$, for all $x_{2} y_{2} \in E_{2}$,

(iii) $\left\{\begin{array}{l}\left(\mu_{\mathcal{Q}_{1}} \bullet \mu_{\mathcal{Q}_{2}}\right)\left(\left(x_{1}, x_{2}\right)\left(y_{1}, y_{2}\right)\right)=\mu_{\mathcal{P}_{1}}\left(x_{1} y_{1}\right) \wedge \mu_{\mathcal{Q}_{2}}\left(x_{2} y_{2}\right) \\ \left(v_{\mathcal{Q}_{1}} \bullet v_{\mathcal{Q}_{2}}\right)\left(\left(x_{1}, x_{2}\right)\left(y_{1}, y_{2}\right)\right)=v_{\mathcal{P}_{1}}\left(x_{1} y_{1}\right) \vee v_{\mathcal{Q}_{2}}\left(x_{2} y_{2}\right) \text { for all } x_{1} y_{1} \in E_{1} \text {, for all } x_{2} y_{2} \in E_{2} .\end{array}\right.$

Proposition 3. Let $\mathcal{G}_{1}$ and $\mathcal{G}_{2}$ be the PFGs of the graphs $G_{1}$ and $G_{2}$, respectively. The semi-strong product $\mathcal{G}_{1} \bullet \mathcal{G}_{2}$ of $\mathcal{G}_{1}$ and $\mathcal{G}_{2}$ is a PFG of $G_{1} \bullet G_{2}$.

Definition 11. Let $\mathcal{G}_{1}=\left(\mathcal{P}_{1}, \mathcal{Q}_{1}\right)$ and $\mathcal{G}_{2}=\left(\mathcal{P}_{2}, \mathcal{Q}_{2}\right)$ be two PFGs. For any vertex $\left(x_{1}, x_{2}\right) \in V_{1} \times V_{2}$,

$$
\begin{aligned}
\left(d_{\mu}\right)_{\mathcal{G}_{1} \bullet \mathcal{G}_{2}}\left(x_{1}, x_{2}\right) & =\sum_{\left(x_{1}, x_{2}\right)\left(y_{1}, y_{2}\right) \in E_{1} \bullet E_{2}}\left(\mu_{\mathcal{Q}_{1}} \bullet \mu_{\mathcal{Q}_{2}}\right)\left(\left(x_{1}, x_{2}\right)\left(y_{1}, y_{2}\right)\right) \\
& =\sum_{x_{1}=y_{1}, x_{2} y_{2} \in E_{2}} \mu_{\mathcal{P}_{1}}\left(x_{1}\right) \wedge \mu_{\mathcal{Q}_{2}}\left(x_{2} y_{2}\right)+\sum_{x_{1} y_{1} \in E_{1}, x_{2} y_{2} \in E_{2}} \mu_{\mathcal{Q}_{1}}\left(x_{1} y_{1}\right) \wedge \mu_{\mathcal{Q}_{2}}\left(x_{2} y_{2}\right), \\
\left(d_{v}\right)_{\mathcal{G}_{1} \bullet \mathcal{G}_{2}}\left(x_{1}, x_{2}\right) & =\sum_{\left(x_{1}, x_{2}\right)\left(y_{1}, y_{2}\right) \in E_{1} \bullet E_{2}}\left(v_{\mathcal{Q}_{1}} \bullet v_{\mathcal{Q}_{2}}\right)\left(\left(x_{1}, x_{2}\right)\left(y_{1}, y_{2}\right)\right) \\
& =\sum_{x_{1}=y_{1}, x_{2} y_{2} \in E_{2}} v_{\mathcal{P}_{1}}\left(x_{1}\right) \vee v_{\mathcal{Q}_{2}}\left(x_{2} y_{2}\right)+\sum_{x_{1} y_{1} \in E_{1}, x_{2} y_{2} \in E_{2}} v_{\mathcal{Q}_{1}}\left(x_{1} y_{1}\right) \vee v_{\mathcal{Q}_{2}}\left(x_{2} y_{2}\right) .
\end{aligned}
$$


Theorem 5. Let $\mathcal{G}_{1}=\left(\mathcal{P}_{1}, \mathcal{Q}_{1}\right)$ and $\mathcal{G}_{2}=\left(\mathcal{P}_{2}, \mathcal{Q}_{2}\right)$ be two PFGs. If $\mu_{\mathcal{P}_{1}} \geq \mu_{\mathcal{Q}_{2}}, v_{\mathcal{P}_{1}} \leq v_{\mathcal{Q}_{2}}, \mu_{\mathcal{Q}_{1}} \leq \mu_{\mathcal{Q}_{2}}$, $v_{\mathcal{Q}_{1}} \geq v_{\mathcal{Q}_{2}}$. Then, $d_{\mathcal{G}_{1}} \bullet \mathcal{G}_{2}\left(x_{1}, x_{2}\right)=d_{\mathcal{G}_{1}}\left(x_{1}\right)+d_{\mathcal{G}_{2}}\left(x_{2}\right)$ for all $\left(x_{1}, x_{2}\right) \in V_{1} \times V_{2}$.

Proof. By definition of vertex degree of $\mathcal{G}_{1} \bullet \mathcal{G}_{2}$, we have

$$
\begin{aligned}
\left(d_{\mu}\right)_{\mathcal{G}_{1} \bullet \mathcal{G}_{2}}\left(x_{1}, x_{2}\right) & =\sum_{\left(x_{1}, x_{2}\right)\left(y_{1}, y_{2}\right) \in E_{1} \bullet E_{2}}\left(\mu_{\mathcal{Q}_{1}} \bullet \mu_{\mathcal{Q}_{2}}\right)\left(\left(x_{1}, x_{2}\right)\left(y_{1}, y_{2}\right)\right) \\
& =\sum_{x_{1}=y_{1}, x_{2} y_{2 \in E_{2}}} \mu_{\mathcal{P}_{1}}\left(x_{1}\right) \wedge \mu_{\mathcal{Q}_{2}}\left(x_{2} y_{2}\right)+\sum_{x_{1} y_{1} \in E_{1}, x_{2} y_{2} \in E_{2}} \mu_{\mathcal{Q}_{1}}\left(x_{1} y_{1}\right) \wedge \mu_{\mathcal{Q}_{2}}\left(x_{2} y_{2}\right) \\
& =\sum_{x_{2} y_{2} \in E_{2}} \mu_{\mathcal{Q}_{2}}\left(x_{2} y_{2}\right)+\sum_{x_{1} y_{1} \in E_{1}} \mu_{\mathcal{Q}_{1}}\left(x_{1} y_{1}\right) \\
& \left(\text { Since } \mu_{\mathcal{P}_{1}} \geq \mu_{\mathcal{Q}_{2}} \text { and } \mu_{\mathcal{Q}_{1}} \leq \mu_{\mathcal{Q}_{2}}\right) \\
= & \left(d_{\mu}\right)_{\mathcal{G}_{2}}\left(x_{2}\right)+\left(d_{\mu}\right)_{\mathcal{G}_{1}}\left(x_{1}\right) .
\end{aligned}
$$

Analogously, it is easy to show that $\left(d_{v}\right)_{\mathcal{G}_{1} \bullet \mathcal{G}_{2}}\left(x_{1}, x_{2}\right)=\left(d_{v}\right)_{\mathcal{G}_{1}}\left(x_{1}\right)+\left(d_{v}\right)_{\mathcal{G}_{2}}\left(x_{2}\right)$. Hence, $d_{\mathcal{G}_{1}} \bullet \mathcal{G}_{2}\left(x_{1}, x_{2}\right)=d_{\mathcal{G}_{1}}\left(x_{1}\right)+d_{\mathcal{G}_{2}}\left(x_{2}\right)$.

Definition 12. Let $\mathcal{G}_{1}=\left(\mathcal{P}_{1}, \mathcal{Q}_{1}\right)$ and $\mathcal{G}_{2}=\left(\mathcal{P}_{2}, \mathcal{Q}_{2}\right)$ be two PFGs. For any vertex $\left(x_{1}, x_{2}\right) \in V_{1} \times V_{2}$,

$$
\begin{aligned}
\left(t d_{\mu}\right)_{\mathcal{G}_{1} \bullet \mathcal{G}_{2}}\left(x_{1}, x_{2}\right)= & \sum_{\left(x_{1}, x_{2}\right)\left(y_{1}, y_{2}\right) \in E_{1} \bullet E_{2}}\left(\mu_{\mathcal{Q}_{1}} \bullet \mu_{\mathcal{Q}_{2}}\right)\left(\left(x_{1}, x_{2}\right)\left(y_{1}, y_{2}\right)\right)+\left(\mu_{\mathcal{P}_{1}} \bullet \mu_{\mathcal{P}_{2}}\right)\left(x_{1}, x_{2}\right) \\
= & \sum_{x_{1}=y_{1}, x_{2} y_{2} \in E_{2}} \mu_{\mathcal{P}_{1}}\left(x_{1}\right) \wedge \mu_{\mathcal{Q}_{2}}\left(x_{2} y_{2}\right)+\sum_{x_{1} y_{1} \in E_{1}, x_{2} y_{2} \in E_{2}} \mu_{\mathcal{Q}_{1}}\left(x_{1} y_{1}\right) \wedge \mu_{\mathcal{Q}_{2}}\left(x_{2} y_{2}\right) \\
& +\mu_{\mathcal{P}_{1}}\left(x_{1}\right) \wedge \mu_{\mathcal{P}_{2}}\left(x_{2}\right), \\
\left(t d_{v}\right)_{\mathcal{G}_{1} \bullet \mathcal{G}_{2}}\left(x_{1}, x_{2}\right)= & \sum_{\left(x_{1}, x_{2}\right)\left(y_{1}, y_{2}\right) \in E_{1} \bullet E_{2}}\left(v_{\mathcal{Q}_{1}} \bullet v_{\mathcal{Q}_{2}}\right)\left(\left(x_{1}, x_{2}\right)\left(y_{1}, y_{2}\right)\right)+\left(v_{\mathcal{P}_{1}} \bullet v_{\mathcal{P}_{2}}\right)\left(x_{1}, x_{2}\right) \\
= & \sum_{x_{1}=y_{1}, x_{2} y_{2 \in E_{2}}} v_{\mathcal{P}_{1}}\left(x_{1}\right) \vee v_{\mathcal{Q}_{2}}\left(x_{2} y_{2}\right)+\sum_{x_{1} y_{1} \in E_{1}, x_{2} y_{2} \in E_{2}} v_{\mathcal{Q}_{1}}\left(x_{1} y_{1}\right) \vee v_{\mathcal{Q}_{2}}\left(x_{2} y_{2}\right) \\
& +v_{\mathcal{P}_{1}}\left(x_{1}\right) \vee v_{\mathcal{P}_{2}}\left(x_{2}\right) .
\end{aligned}
$$

Theorem 6. Let $\mathcal{G}_{1}=\left(\mathcal{P}_{1}, \mathcal{Q}_{1}\right)$ and $\mathcal{G}_{2}=\left(\mathcal{P}_{2}, \mathcal{Q}_{2}\right)$ be two PFGs. If

(i) $\mu_{\mathcal{P}_{1}} \geq \mu_{\mathcal{Q}_{2}}, \mu_{\mathcal{Q}_{1}} \leq \mu_{\mathcal{Q}_{2}}$, then $\left(t d_{\mu}\right)_{\mathcal{G}_{1} \bullet \mathcal{G}_{2}}\left(x_{1}, x_{2}\right)=\left(t d_{\mu}\right)_{\mathcal{G}_{1}}\left(x_{1}\right)+\left(t d_{\mu}\right)_{\mathcal{G}_{2}}\left(x_{2}\right)-\mu_{\mathcal{P}_{1}}\left(x_{1}\right) \vee \mu_{\mathcal{P}_{2}}\left(x_{2}\right)$;

(ii) $v_{\mathcal{P}_{1}} \leq v_{\mathcal{Q}_{2}}, v_{\mathcal{Q}_{1}} \geq v_{\mathcal{Q}_{2}}$, then $\left(t d_{v}\right)_{\mathcal{G}_{1} \bullet \mathcal{G}_{2}}\left(x_{1}, x_{2}\right)=\left(t d_{v}\right)_{\mathcal{G}_{1}}\left(x_{1}\right)+\left(t d_{v}\right)_{\mathcal{G}_{2}}\left(x_{2}\right)-v_{\mathcal{P}_{1}}\left(x_{1}\right) \wedge v_{\mathcal{P}_{2}}\left(x_{2}\right)$

for all $\left(x_{1}, x_{2}\right) \in V_{1} \times V_{2}$.

Proof. By definition of vertex total degree of $\mathcal{G}_{1} \bullet \mathcal{G}_{2}$,

(i) If $\mu_{\mathcal{P}_{1}} \geq \mu_{\mathcal{Q}_{2}}, \mu_{\mathcal{Q}_{1}} \leq \mu_{\mathcal{Q}_{2}}$

$$
\begin{aligned}
\left(t d_{\mu}\right)_{\mathcal{G}_{1}} \bullet \mathcal{G}_{2}\left(x_{1}, x_{2}\right)= & \sum_{x_{1}=y_{1}, x_{2} y_{2} \in E_{2}} \mu_{\mathcal{P}_{1}}\left(x_{1}\right) \wedge \mu_{\mathcal{Q}_{2}}\left(x_{2} y_{2}\right)+\sum_{x_{1} y_{1} \in E_{1}, x_{2} y_{2} \in E_{2}} \mu_{\mathcal{Q}_{1}}\left(x_{1} y_{1}\right) \wedge \mu_{\mathcal{Q}_{2}}\left(x_{2} y_{2}\right) \\
& +\mu_{\mathcal{P}_{1}}\left(x_{1}\right) \wedge \mu_{\mathcal{P}_{2}}\left(x_{2}\right) \\
= & \sum_{x_{2} y_{2} \in E_{2}} \mu_{\mathcal{Q}_{2}}\left(x_{2} y_{2}\right)+\sum_{x_{1} y_{1} \in E_{1}} \mu_{\mathcal{Q}_{1}}\left(x_{1} y_{1}\right)+\mu_{\mathcal{P}_{1}}\left(x_{1}\right)+\mu_{\mathcal{P}_{2}}\left(x_{2}\right) \\
& -\mu_{\mathcal{P}_{1}}\left(x_{1}\right) \vee \mu_{\mathcal{P}_{2}}\left(x_{2}\right) \\
= & \left(t d_{\mu}\right)_{\mathcal{G}_{1}}\left(x_{1}\right)+\left(t d_{\mu}\right)_{\mathcal{G}_{2}}\left(x_{2}\right)-\mu_{\mathcal{P}_{1}}\left(x_{1}\right) \vee \mu_{\mathcal{P}_{2}}\left(x_{2}\right) .
\end{aligned}
$$

Analogously, we can prove (ii).

Example 4. Consider two PFGs $\mathcal{G}_{1}$ and $\mathcal{G}_{2}$ as given in Example 2, where $\mu_{\mathcal{P}_{1}} \geq \mu_{\mathcal{Q}_{2}}, v_{\mathcal{P}_{1}} \leq v_{\mathcal{Q}_{2}}, \mu_{\mathcal{Q}_{1}} \leq \mu_{\mathcal{Q}_{2}}$, $v_{\mathcal{Q}_{1}} \geq v_{\mathcal{Q}_{2}}$, and their semi-strong product $\mathcal{G}_{1} \bullet \mathcal{G}_{2}$ is shown in Figure 6 .

Thus, by Theorem 5, we have 


$$
\begin{array}{r}
\left(d_{\mu}\right)_{\mathcal{G}_{1} \bullet \mathcal{G}_{2}}(m, p)=\left(d_{\mu}\right)_{\mathcal{G}_{1}}(m)+\left(d_{\mu}\right)_{\mathcal{G}_{2}}(p)=1.7 \\
\left(d_{v}\right)_{\mathcal{G}_{1} \bullet \mathcal{G}_{2}}(m, p)=\left(d_{v}\right)_{\mathcal{G}_{1}}(m)+\left(d_{v}\right)_{\mathcal{G}_{2}}(p)=2.1 .
\end{array}
$$

Therefore, $d_{\mathcal{G}_{1}} \bullet \mathcal{G}_{2}(m, p)=(1.7,2.1)$.

In addition, by Theorem 6, we have

$$
\begin{aligned}
\left(t d_{\mu}\right)_{\mathcal{G}_{1} \bullet \mathcal{G}_{2}}(m, p) & =\left(t d_{\mu}\right)_{\mathcal{G}_{1}}(m)+\left(t d_{\mu}\right)_{\mathcal{G}_{2}}(p)-\mu_{\mathcal{P}_{1}}(m) \vee \mu_{\mathcal{P}_{2}}(p)=2.5 \\
\left(t d_{v}\right)_{\mathcal{G}_{1}} \bullet \mathcal{G}_{2}(m, p) & =\left(t d_{v}\right)_{\mathcal{G}_{1}}(m)+\left(t d_{v}\right)_{\mathcal{G}_{2}}(p)-v_{\mathcal{P}_{1}}(m) \wedge v_{\mathcal{P}_{2}}(p)=2.7 .
\end{aligned}
$$

Therefore, $t d_{\mathcal{G}_{1} \bullet \mathcal{G}_{2}}(m, p)=(2.5,2.7)$.

Similarly, we can find the degree and total degree of all the vertices in $\mathcal{G}_{1} \bullet \mathcal{G}_{2}$.

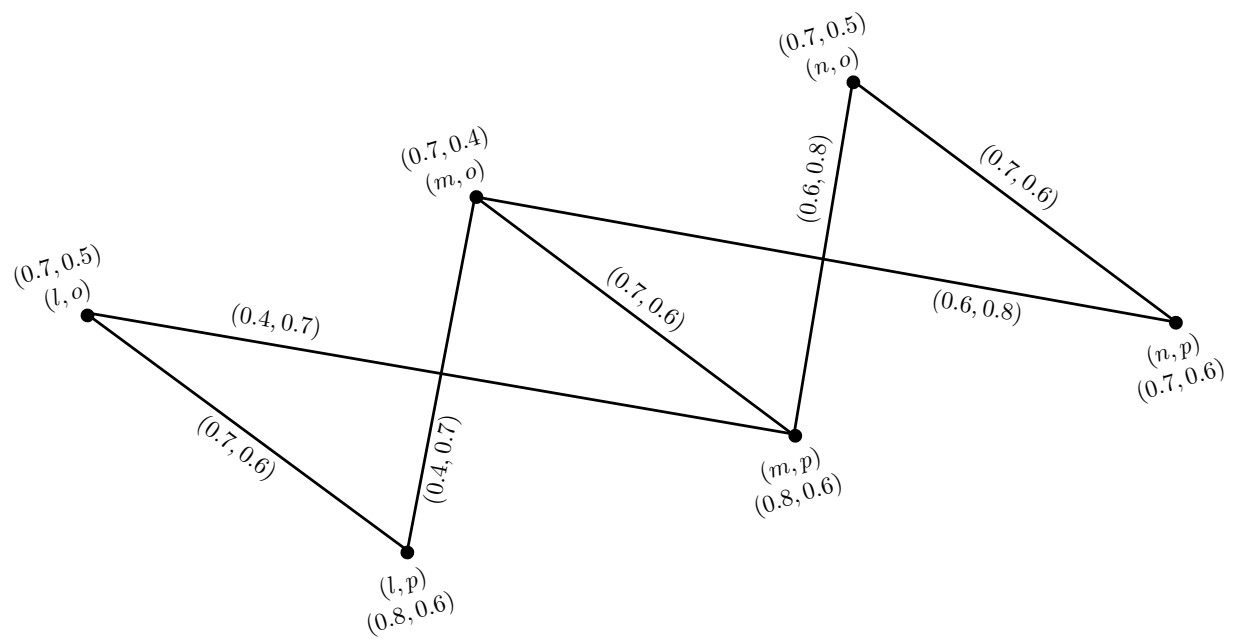

Figure 6. Semi-strong product of two PFGs.

Definition 13. Let $\mathcal{G}_{1}=\left(\mathcal{P}_{1}, \mathcal{Q}_{1}\right)$ and $\mathcal{G}_{2}=\left(\mathcal{P}_{2}, \mathcal{Q}_{2}\right)$ be two PFGs of $G_{1}=\left(V_{1}, E_{1}\right)$ and $G_{2}=\left(V_{2}, E_{2}\right)$, respectively. The strong product of these two PFGs is denoted by $\mathcal{G}_{1} \otimes \mathcal{G}_{2}=\left(\mathcal{P}_{1} \otimes \mathcal{P}_{2}, \mathcal{Q}_{1} \otimes \mathcal{Q}_{2}\right)$ and defined as:

(i) $\left\{\begin{array}{l}\left(\mu_{\mathcal{P}_{1}} \otimes \mu_{\mathcal{P}_{2}}\right)\left(x_{1}, x_{2}\right)=\mu_{\mathcal{P}_{1}}\left(x_{1}\right) \wedge \mu_{\mathcal{P}_{2}}\left(x_{2}\right) \\ \left(v_{\mathcal{P}_{1}} \otimes v_{\mathcal{P}_{2}}\right)\left(x_{1}, x_{2}\right)=v_{\mathcal{P}_{1}}\left(x_{1}\right) \vee v_{\mathcal{P}_{2}}\left(x_{2}\right) \quad \text { for all }\left(x_{1}, x_{2}\right) \in V_{1} \times V_{2},\end{array}\right.$

(ii) $\left\{\begin{array}{l}\left(\mu_{\mathcal{Q}_{1}} \otimes \mu_{\mathcal{Q}_{2}}\right)\left(\left(x, x_{2}\right)\left(x, y_{2}\right)\right)=\mu_{\mathcal{P}_{1}}(x) \wedge \mu_{\mathcal{Q}_{2}}\left(x_{2} y_{2}\right) \\ \left(v_{\mathcal{Q}_{1}} \otimes v_{\mathcal{Q}_{2}}\right)\left(\left(x, x_{2}\right)\left(x, y_{2}\right)\right)=v_{\mathcal{P}_{1}}(x) \vee v_{\mathcal{Q}_{2}}\left(x_{2} y_{2}\right) \quad \text { for all } x \in V_{1}, \text { for all } x_{2} y_{2} \in E_{2},\end{array}\right.$

(iii) $\left\{\begin{array}{l}\left(\mu_{\mathcal{Q}_{1}} \otimes \mu_{\mathcal{Q}_{2}}\right)\left(\left(x_{1}, z\right)\left(y_{1}, z\right)\right)=\mu_{\mathcal{Q}_{1}}\left(x_{1} y_{1}\right) \wedge \mu_{\mathcal{P}_{2}}(z) \\ \left(v_{\mathcal{Q}_{1}} \otimes v_{\mathcal{Q}_{2}}\right)\left(\left(x_{1}, z\right)\left(y_{1}, z\right)\right)=v_{\mathcal{Q}_{1}}\left(x_{1} y_{1}\right) \vee v_{\mathcal{P}_{2}}(z) \quad \text { for all } z \in V_{2}, \text { for all } x_{1} y_{1} \in E_{1},\end{array}\right.$

(iv) $\left\{\begin{array}{l}\left(\mu_{\mathcal{Q}_{1}} \otimes \mu_{\mathcal{Q}_{2}}\right)\left(\left(x_{1}, x_{2}\right)\left(y_{1}, y_{2}\right)\right)=\mu_{\mathcal{Q}_{1}}\left(x_{1} y_{1}\right) \wedge \mu_{\mathcal{Q}_{2}}\left(x_{2} y_{2}\right) \\ \left(v_{\mathcal{Q}_{1}} \otimes v_{\mathcal{Q}_{2}}\right)\left(\left(x_{1}, x_{2}\right)\left(y_{1}, y_{2}\right)\right)=v_{\mathcal{Q}_{1}}\left(x_{1} y_{1}\right) \vee v_{\mathcal{Q}_{2}}\left(x_{2} y_{2}\right) \quad \text { for all } x_{1} y_{1} \in E_{1}, \text { for all } x_{2} y_{2} \in E_{2} .\end{array}\right.$

Proposition 4. Let $\mathcal{G}_{1}$ and $\mathcal{G}_{2}$ be the PFGs of the graphs $G_{1}$ and $G_{2}$, respectively. The strong product $\mathcal{G}_{1} \otimes \mathcal{G}_{2}$ of $\mathcal{G}_{1}$ and $\mathcal{G}_{2}$ is a PFG of $G_{1} \otimes G_{2}$.

Definition 14. Let $\mathcal{G}_{1}=\left(\mathcal{P}_{1}, \mathcal{Q}_{1}\right)$ and $\mathcal{G}_{2}=\left(\mathcal{P}_{2}, \mathcal{Q}_{2}\right)$ be two PFGs. For any vertex $\left(x_{1}, x_{2}\right) \in V_{1} \times V_{2}$,

$$
\begin{aligned}
\left(d_{\mu}\right)_{\mathcal{G}_{1} \otimes \mathcal{G}_{2}}\left(x_{1}, x_{2}\right)= & \sum_{\left(x_{1}, x_{2}\right)\left(y_{1}, y_{2}\right) \in E_{1} \otimes E_{2}}\left(\mu_{\mathcal{Q}_{1}} \otimes \mu_{\mathcal{Q}_{2}}\right)\left(\left(x_{1}, x_{2}\right)\left(y_{1}, y_{2}\right)\right) \\
= & \sum_{x_{1}=y_{1}, x_{2} y_{2} \in E_{2}} \mu_{\mathcal{P}_{1}}\left(x_{1}\right) \wedge \mu_{\mathcal{Q}_{2}}\left(x_{2} y_{2}\right)+\sum_{x_{2}=y_{2}, x_{1} y_{1} \in E_{1}} \mu_{\mathcal{P}_{2}}\left(x_{2}\right) \wedge \mu_{\mathcal{Q}_{1}}\left(x_{1} y_{1}\right) \\
& +\sum_{x_{1} y_{1} \in E_{1}, x_{2} y_{2} \in E_{2}} \mu_{\mathcal{Q}_{1}}\left(x_{1} y_{1}\right) \wedge \mu_{\mathcal{Q}_{2}}\left(x_{2} y_{2}\right)
\end{aligned}
$$




$$
\begin{aligned}
\left(d_{v}\right)_{\mathcal{G}_{1} \otimes \mathcal{G}_{2}}\left(x_{1}, x_{2}\right)= & \sum_{\left(x_{1}, x_{2}\right)\left(y_{1}, y_{2}\right) \in E_{1} \otimes E_{2}}\left(v_{\mathcal{Q}_{1}} \otimes v_{\mathcal{Q}_{2}}\right)\left(\left(x_{1}, x_{2}\right)\left(y_{1}, y_{2}\right)\right) \\
= & \sum_{x_{1}=y_{1}, x_{2} y_{2} \in E_{2}} v_{\mathcal{P}_{1}}\left(x_{1}\right) \vee v_{\mathcal{Q}_{2}}\left(x_{2} y_{2}\right)+\sum_{x_{2}=y_{2}, x_{1} y_{1} \in E_{1}} v_{\mathcal{P}_{2}}\left(x_{2}\right) \vee v_{\mathcal{Q}_{1}}\left(x_{1} y_{1}\right) \\
& +\sum_{x_{1} y_{1} \in E_{1}, x_{2} y_{2} \in E_{2}} v_{\mathcal{Q}_{1}}\left(x_{1} y_{1}\right) \vee v_{\mathcal{Q}_{2}}\left(x_{2} y_{2}\right) .
\end{aligned}
$$

Theorem 7. Let $\mathcal{G}_{1}=\left(\mathcal{P}_{1}, \mathcal{Q}_{1}\right)$ and $\mathcal{G}_{2}=\left(\mathcal{P}_{2}, \mathcal{Q}_{2}\right)$ be two PFGs. If $\mu_{\mathcal{P}_{1}} \geq \mu_{\mathcal{Q}_{2}}, v_{\mathcal{P}_{1}} \leq v_{\mathcal{Q}_{2}}, \mu_{\mathcal{P}_{2}} \geq \mu_{\mathcal{Q}_{1}}$, $v_{\mathcal{P}_{2}} \leq v_{\mathcal{Q}_{1}}, \mu_{\mathcal{Q}_{1}} \leq \mu_{\mathcal{Q}_{2}}, v_{\mathcal{Q}_{1}} \geq v_{\mathcal{Q}_{2}}$. Then, for all $\left(x_{1}, x_{2}\right) \in V_{1} \times V_{2}, d_{\mathcal{G}_{1} \otimes \mathcal{G}_{2}}\left(x_{1}, x_{2}\right)=p_{2} d_{\mathcal{G}_{1}}\left(x_{1}\right)+$ $d_{\mathcal{G}_{2}}\left(x_{2}\right)$, where $p_{2}=\left|V_{2}\right|$.

Proof. By definition of vertex degree of $\mathcal{G}_{1} \otimes \mathcal{G}_{2}$, we have

$$
\begin{aligned}
\left(d_{\mu}\right)_{\mathcal{G}_{1} \otimes \mathcal{G}_{2}}\left(x_{1}, x_{2}\right)= & \sum_{\left(x_{1}, x_{2}\right)\left(y_{1}, y_{2}\right) \in E_{1} \otimes E_{2}}\left(\mu_{\mathcal{Q}_{1}} \otimes \mu_{\mathcal{Q}_{2}}\right)\left(\left(x_{1}, x_{2}\right)\left(y_{1}, y_{2}\right)\right) \\
= & \sum_{x_{1}=y_{1}, x_{2} y_{2} \in E_{2}} \mu_{\mathcal{P}_{1}}\left(x_{1}\right) \wedge \mu_{\mathcal{Q}_{2}}\left(x_{2} y_{2}\right)+\sum_{x_{2}=y_{2}, x_{1} y_{1} \in E_{1}} \mu_{\mathcal{P}_{2}}\left(x_{2}\right) \wedge \mu_{\mathcal{Q}_{1}}\left(x_{1} y_{1}\right) \\
& +\sum_{x_{1} y_{1} \in E_{1}, x_{2} y_{2} \in E_{2}} \mu_{\mathcal{Q}_{1}}\left(x_{1} y_{1}\right) \wedge \mu_{\mathcal{Q}_{2}}\left(x_{2} y_{2}\right) \\
= & \sum_{x_{2} y_{2} \in E_{2}} \mu_{\mathcal{Q}_{2}}\left(x_{2} y_{2}\right)+\sum_{x_{1} y_{1} \in E_{1}} \mu_{\mathcal{Q}_{1}}\left(x_{1} y_{1}\right)+\sum_{x_{1} y_{1} \in E_{1}} \mu_{\mathcal{Q}_{1}}\left(x_{1} y_{1}\right) \\
& \left(\text { Since } \mu_{\mathcal{P}_{1}} \geq \mu_{\mathcal{Q}_{2}}, \mu_{\mathcal{P}_{2}} \geq \mu_{\mathcal{Q}_{1}} \text { and } \mu_{\mathcal{Q}_{1}} \leq \mu_{\mathcal{Q}_{2}}\right) \\
= & p_{2}\left(d_{\mu}\right)_{\mathcal{G}_{1}}\left(x_{1}\right)+\left(d_{\mu}\right)_{\mathcal{G}_{2}}\left(x_{2}\right) .
\end{aligned}
$$

Analogously, it is easy to show that $\left(d_{v}\right)_{\mathcal{G}_{1} \bowtie \mathcal{G}_{2}}\left(x_{1}, x_{2}\right)=p_{2}\left(d_{v}\right)_{\mathcal{G}_{1}}\left(x_{1}\right)+\left(d_{v}\right)_{\mathcal{G}_{2}}\left(x_{2}\right)$. Hence, $d_{\mathcal{G}_{1} \otimes \mathcal{G}_{2}}\left(x_{1}, x_{2}\right)=p_{2} d_{\mathcal{G}_{1}}\left(x_{1}\right)+d_{\mathcal{G}_{2}}\left(x_{2}\right)$.

Definition 15. Let $\mathcal{G}_{1}=\left(\mathcal{P}_{1}, \mathcal{Q}_{1}\right)$ and $\mathcal{G}_{2}=\left(\mathcal{P}_{2}, \mathcal{Q}_{2}\right)$ be two PFGs. For any vertex $\left(x_{1}, x_{2}\right) \in V_{1} \times V_{2}$,

$$
\begin{aligned}
\left(t d_{\mu}\right)_{\mathcal{G}_{1} \otimes \mathcal{G}_{2}}\left(x_{1}, x_{2}\right)= & \sum_{\left(x_{1}, x_{2}\right)\left(y_{1}, y_{2}\right) \in E_{1} \otimes E_{2}}\left(\mu_{\mathcal{Q}_{1}} \otimes \mu_{\mathcal{Q}_{2}}\right)\left(\left(x_{1}, x_{2}\right)\left(y_{1}, y_{2}\right)\right)+\left(\mu_{\mathcal{P}_{1}} \otimes \mu_{\mathcal{P}_{2}}\right)\left(x_{1}, x_{2}\right) \\
= & \sum_{x_{1}=y_{1}, x_{2} y_{2} \in E_{2}} \mu_{\mathcal{P}_{1}}\left(x_{1}\right) \wedge \mu_{\mathcal{Q}_{2}}\left(x_{2} y_{2}\right)+\sum_{x_{2}=y_{2}, x_{1} y_{1} \in E_{1}} \mu_{\mathcal{P}_{2}}\left(x_{2}\right) \wedge \mu_{\mathcal{Q}_{1}}\left(x_{1} y_{1}\right) \\
& +\sum_{x_{1} y_{1} \in E_{1}, x_{2} y_{2} \in E_{2}} \mu_{\mathcal{Q}_{1}}\left(x_{1} y_{1}\right) \wedge \mu_{\mathcal{Q}_{2}}\left(x_{2} y_{2}\right)+\mu_{\mathcal{P}_{1}}\left(x_{1}\right) \wedge \mu_{\mathcal{P}_{2}}\left(x_{2}\right), \\
\left(t d_{v}\right)_{\mathcal{G}_{1} \otimes \mathcal{G}_{2}}\left(x_{1}, x_{2}\right)= & \sum_{\left(x_{1}, x_{2}\right)\left(y_{1}, y_{2}\right) \in E_{1} \otimes E_{2}}\left(v_{\mathcal{Q}_{1}} \otimes v_{\mathcal{Q}_{2}}\right)\left(\left(x_{1}, x_{2}\right)\left(y_{1}, y_{2}\right)\right)+\left(v_{\mathcal{P}_{1}} \otimes v_{\mathcal{P}_{2}}\right)\left(x_{1}, x_{2}\right) \\
= & \sum_{x_{1}=y_{1}, x_{2} y_{2} \in E_{2}} v_{\mathcal{P}_{1}}\left(x_{1}\right) \vee v_{\mathcal{Q}_{2}}\left(x_{2} y_{2}\right)+\sum_{x_{2}=y_{2}, x_{1} y_{1} \in E_{1}} v_{\mathcal{P}_{2}}\left(x_{2}\right) \vee v_{\mathcal{Q}_{1}}\left(x_{1} y_{1}\right) \\
& +\sum_{x_{1} y_{1} \in E_{1}, x_{2} y_{2} \in E_{2}} v_{\mathcal{Q}_{1}}\left(x_{1} y_{1}\right) \vee v_{\mathcal{Q}_{2}}\left(x_{2} y_{2}\right)+v_{\mathcal{P}_{1}}\left(x_{1}\right) \vee v_{\mathcal{P}_{2}}\left(x_{2}\right) .
\end{aligned}
$$

Theorem 8. Let $\mathcal{G}_{1}=\left(\mathcal{P}_{1}, \mathcal{Q}_{1}\right)$ and $\mathcal{G}_{2}=\left(\mathcal{P}_{2}, \mathcal{Q}_{2}\right)$ be two PFGs. If

(i) $\mu_{\mathcal{P}_{1}} \geq \mu_{\mathcal{Q}_{2}}, \mu_{\mathcal{P}_{2}} \geq \mu_{\mathcal{Q}_{1}}, \mu_{\mathcal{Q}_{1}} \leq \mu_{\mathcal{Q}_{2}}$, then $(t d \mu)_{\mathcal{G}_{1} \otimes \mathcal{G}_{2}}\left(x_{1}, x_{2}\right)=\left(t d_{\mu}\right)_{\mathcal{G}_{2}}\left(x_{2}\right)+p_{2}\left(t d_{\mu}\right)_{\mathcal{G}_{1}}\left(x_{1}\right)-$ $\left(p_{2}-1\right) \mu_{\mathcal{P}_{1}}\left(x_{1}\right)-\mu_{\mathcal{P}_{1}}\left(x_{1}\right) \vee \mu_{\mathcal{P}_{2}}\left(x_{2}\right)$

(ii) $v_{\mathcal{P}_{1}} \leq v_{\mathcal{Q}_{2}}, v_{\mathcal{P}_{2}} \leq v_{\mathcal{Q}_{1}}, \mu_{\mathcal{Q}_{1}} \geq \mu_{\mathcal{Q}_{2}}$, then $\left(t d_{v}\right)_{\mathcal{G}_{1} \otimes \mathcal{G}_{2}}\left(x_{1}, x_{2}\right)=\left(t d_{v}\right)_{\mathcal{G}_{2}}\left(x_{2}\right)+p_{2}\left(t d_{v}\right)_{\mathcal{G}_{1}}\left(x_{1}\right)-$ $\left(p_{2}-1\right) v_{\mathcal{P}_{1}}\left(x_{1}\right)-v_{\mathcal{P}_{1}}\left(x_{1}\right) \wedge v_{\mathcal{P}_{2}}\left(x_{2}\right)$

for all $\left(x_{1}, x_{2}\right) \in V_{1} \times V_{2}$.

Proof. For any vertex $\left(x_{1}, x_{2}\right) \in V_{1} \times V_{2}$ ， 
(i) If $\mu_{\mathcal{P}_{1}} \geq \mu_{\mathcal{Q}_{2}}, \mu_{\mathcal{P}_{2}} \geq \mu_{\mathcal{Q}_{1}}, \mu_{\mathcal{Q}_{1}} \leq \mu_{\mathcal{Q}_{2}}$

$$
\begin{aligned}
\left(t d_{\mu}\right)_{\mathcal{G}_{1} \otimes \mathcal{G}_{2}}\left(x_{1}, x_{2}\right)= & \sum_{x_{1}=y_{1}, x_{2} y_{2 \in E_{2}}} \mu_{\mathcal{P}_{1}}\left(x_{1}\right) \wedge \mu_{\mathcal{Q}_{2}}\left(x_{2} y_{2}\right)+\sum_{x_{2}=y_{2}, x_{1} y_{1} \in E_{1}} \mu_{\mathcal{P}_{2}}\left(x_{2}\right) \wedge \mu_{\mathcal{Q}_{1}}\left(x_{1} y_{1}\right) \\
& +\sum_{x_{1} y_{1} \in E_{1}, x_{2} y_{2} \in E_{2}} \mu_{\mathcal{Q}_{1}}\left(x_{1} y_{1}\right) \wedge \mu_{\mathcal{Q}_{2}}\left(x_{2} y_{2}\right)+\mu_{\mathcal{P}_{1}}\left(x_{1}\right) \wedge \mu_{\mathcal{P}_{2}}\left(x_{2}\right) \\
= & \sum_{x_{2} y_{2} \in E_{2}} \mu_{\mathcal{Q}_{2}}\left(x_{2} y_{2}\right)+\sum_{x_{1} y_{1} \in E_{1}} \mu_{\mathcal{Q}_{1}}\left(x_{1} y_{1}\right)+\sum_{x_{1} y_{1} \in E_{1}} \mu_{\mathcal{Q}_{1}}\left(x_{1} y_{1}\right) \\
= & +\mu_{\mathcal{P}_{1}}\left(x_{1}\right)+\mu_{\mathcal{P}_{2}}\left(x_{2}\right)-\mu_{\mathcal{P}_{1}}\left(x_{1}\right) \vee \mu_{\mathcal{P}_{2}}\left(x_{2}\right) \\
= & \sum_{x_{2} y_{2} \in E_{2}} \mu_{\mathcal{Q}_{2}}\left(x_{2} y_{2}\right)+\mu_{\mathcal{P}_{2}}\left(x_{2}\right)+p_{2} \sum_{x_{1} y_{1} \in E_{1}} \mu_{\mathcal{Q}_{1}}\left(x_{1} y_{1}\right)+\mu_{\mathcal{P}_{1}}\left(x_{1}\right) \\
& -\mu_{\mathcal{P}_{1}}\left(x_{1}\right) \vee \mu_{\mathcal{P}_{2}}\left(x_{2}\right) \\
= & \sum_{x_{2} y_{2} \in E_{2}} \mu_{\mathcal{Q}_{2}}\left(x_{2} y_{2}\right)+\mu_{\mathcal{P}_{2}}\left(x_{2}\right)+p_{2}\left(\sum_{x_{1} y_{1} \in E_{1}} \mu_{\mathcal{Q}_{1}}\left(x_{1} y_{1}\right)+\mu_{\mathcal{P}_{1}}\left(x_{1}\right)\right) \\
& -\left(p_{2}-1\right) \mu_{\mathcal{P}_{1}}\left(x_{1}\right)-\mu_{\mathcal{P}_{1}}\left(x_{1}\right) \vee \mu_{\mathcal{P}_{2}}\left(x_{2}\right) \\
= & \left(t d_{\mu}\right)_{\mathcal{G}_{2}}\left(x_{2}\right)+p_{2}\left(t d_{\mu}\right) \mathcal{G}_{1}\left(x_{1}\right)-\left(p_{2}-1\right) \mu_{\mathcal{P}_{1}}\left(x_{1}\right)-\mu_{\mathcal{P}_{1}}\left(x_{1}\right) \vee \mu_{\mathcal{P}_{2}}\left(x_{2}\right) .
\end{aligned}
$$

Analogously, we can prove (ii).

Example 5. Consider two PFGs $\mathcal{G}_{1}$ and $\mathcal{G}_{2}$ as in Example 2, where $\mu_{\mathcal{P}_{1}} \geq \mu_{\mathcal{Q}_{2}}, v_{\mathcal{P}_{1}} \leq v_{\mathcal{Q}_{2}}, \mu_{\mathcal{P}_{2}} \geq \mu_{\mathcal{Q}_{1}}$, $v_{\mathcal{P}_{2}} \leq v_{\mathcal{Q}_{1}}, \mu_{\mathcal{Q}_{1}} \leq \mu_{\mathcal{Q}_{2}}, v_{\mathcal{Q}_{1}} \geq v_{\mathcal{Q}_{2}}$ and their strong product $\mathcal{G}_{1} \otimes \mathcal{G}_{2}$ is shown in Figure 7 .

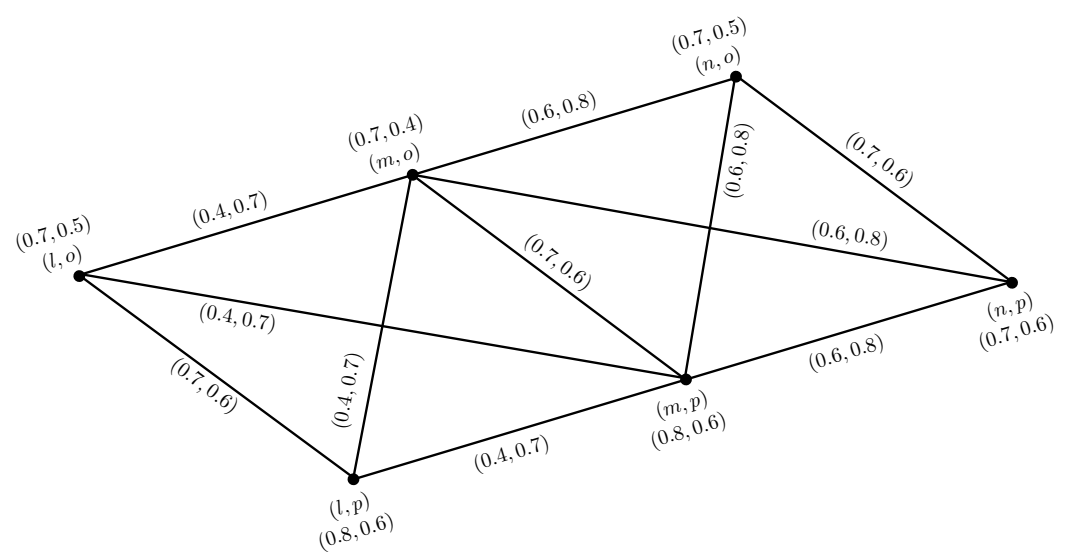

Figure 7. Strong product of two PFGs.

Then, by Theorem 7, we must have

$$
\begin{array}{r}
\left(d_{\mu}\right)_{\mathcal{G}_{1} \otimes \mathcal{G}_{2}}(m, o)=p_{2}\left(d_{\mu}\right)_{\mathcal{G}_{1}}(m)+\left(d_{\mu}\right)_{\mathcal{G}_{2}}(o)=2.7, \\
\left(d_{v}\right)_{\mathcal{G}_{1} \otimes \mathcal{G}_{2}}(m, o)=p_{2}\left(d_{v}\right)_{\mathcal{G}_{1}}(m)+\left(d_{v}\right)_{\mathcal{G}_{2}}(o)=3.6 .
\end{array}
$$

Therefore, $d_{\mathcal{G}_{1} \bowtie \mathcal{G}_{2}}(m, p)=(2.7,3.6)$.

In addition, by Theorem 8 , we must have

$$
\begin{aligned}
& \left(t d_{\mu}\right)_{\mathcal{G}_{1} \otimes \mathcal{G}_{2}}(m, o)=p_{2}\left(t d_{\mu}\right)_{\mathcal{G}_{1}}(m)+\left(t d_{\mu}\right)_{\mathcal{G}_{2}}(o)-\left(p_{2}-1\right) \mu_{\mathcal{P}_{1}}(m)-\mu_{\mathcal{P}_{1}}(m) \vee \mu_{\mathcal{P}_{2}}(o)=3.4 \\
& \left(t d_{v}\right)_{\mathcal{G}_{1} \otimes \mathcal{G}_{2}}(m, o)=p_{2}\left(t d_{v}\right)_{\mathcal{G}_{1}}(m)+\left(t d_{v}\right)_{\mathcal{G}_{2}}(o)-\left(p_{2}-1\right) v_{\mathcal{P}_{1}}(m)-v_{\mathcal{P}_{1}}(m) \wedge v_{\mathcal{P}_{2}}(o)=4.0
\end{aligned}
$$

Therefore, $t d_{\mathcal{G}_{1} \otimes \mathcal{G}_{2}}(m, o)=(3.4,4.0)$.

Similarly, we can find the degree and total degree of all the vertices in $\mathcal{G}_{1} \otimes \mathcal{G}_{2}$. 
Definition 16. Let $\mathcal{G}_{1}=\left(\mathcal{P}_{1}, \mathcal{Q}_{1}\right)$ and $\mathcal{G}_{2}=\left(\mathcal{P}_{2}, \mathcal{Q}_{2}\right)$ be two PFGs of $G_{1}=\left(V_{1}, E_{1}\right)$ and $G_{2}=\left(V_{2}, E_{2}\right)$, respectively. The lexicographic product of these two PFGs is denoted by $\mathcal{G}_{1} \circ \mathcal{G}_{2}=\left(\mathcal{P}_{1} \circ \mathcal{P}_{2}, \mathcal{Q}_{1} \circ \mathcal{Q}_{2}\right)$ and defined as follows:

(i) $\left\{\begin{array}{l}\left(\mu_{\mathcal{P}_{1}} \circ \mu_{\mathcal{P}_{2}}\right)\left(x_{1}, x_{2}\right)=\mu_{\mathcal{P}_{1}}\left(x_{1}\right) \wedge \mu_{\mathcal{P}_{2}}\left(x_{2}\right) \\ \left(v_{\mathcal{P}_{1}} \circ v_{\mathcal{P}_{2}}\right)\left(x_{1}, x_{2}\right)=v_{\mathcal{P}_{1}}\left(x_{1}\right) \vee v_{\mathcal{P}_{2}}\left(x_{2}\right) \quad \text { for all }\left(x_{1}, x_{2}\right) \in V_{1} \times V_{2},\end{array}\right.$

(ii) $\left\{\begin{array}{l}\left(\mu_{\mathcal{Q}_{1}} \circ \mu_{\mathcal{Q}_{2}}\right)\left(\left(x, x_{2}\right)\left(x, y_{2}\right)\right)=\mu_{\mathcal{P}_{1}}(x) \wedge \mu_{\mathcal{Q}_{2}}\left(x_{2} y_{2}\right) \\ \left(v_{\mathcal{Q}_{1}} \circ v_{\mathcal{Q}_{2}}\right)\left(\left(x, x_{2}\right)\left(x, y_{2}\right)\right)=v_{\mathcal{P}_{1}}(x) \vee v_{\mathcal{Q}_{2}}\left(x_{2} y_{2}\right)\end{array} \quad\right.$ for all $x \in V_{1}$, for all $x_{2} y_{2} \in E_{2}$,

(iii) $\left\{\begin{array}{l}\left(\mu_{\mathcal{Q}_{1}} \circ \mu_{\mathcal{Q}_{2}}\right)\left(\left(x_{1}, z\right)\left(y_{1}, z\right)\right)=\mu_{\mathcal{Q}_{1}}\left(x_{1} y_{1}\right) \wedge \mu_{\mathcal{P}_{2}}(z) \\ \left(v_{\mathcal{Q}_{1}} \circ v_{\mathcal{Q}_{2}}\right)\left(\left(x_{1}, z\right)\left(y_{1}, z\right)\right)=v_{\mathcal{Q}_{1}}\left(x_{1} y_{1}\right) \vee v_{\mathcal{P}_{2}}(z) \quad \text { for all } z \in V_{2}, \text { for all } x_{1} y_{1} \in E_{1},\end{array}\right.$

(iv) $\left\{\begin{array}{l}\left(\mu_{\mathcal{Q}_{1}} \circ \mu_{\mathcal{Q}_{2}}\right)\left(\left(x_{1}, x_{2}\right)\left(y_{1}, y_{2}\right)\right)=\mu_{\mathcal{P}_{2}}\left(x_{2}\right) \wedge \mu_{\mathcal{P}_{2}}\left(y_{2}\right) \wedge \mu_{\mathcal{Q}_{1}}\left(x_{1} y_{1}\right) \\ \left(v_{\mathcal{Q}_{1}} \circ v_{\mathcal{Q}_{2}}\right)\left(\left(x_{1}, x_{2}\right)\left(y_{1}, y_{2}\right)\right)=v_{\mathcal{P}_{2}}\left(x_{2}\right) \vee v_{\mathcal{P}_{2}}\left(y_{2}\right) \vee v_{\mathcal{Q}_{1}}\left(x_{1} y_{1}\right) \text { for all } x_{1} y_{1} \in E_{1}, x_{2} \neq y_{2} .\end{array}\right.$

Proposition 5. The lexicographic product $\mathcal{G}_{1}\left[\mathcal{G}_{2}\right]$ of two PFGs of $G_{1}$ and $G_{2}$ is a PFG of $G_{1}\left[G_{2}\right]$.

Definition 17. Let $\mathcal{G}_{1}=\left(\mathcal{P}_{1}, \mathcal{Q}_{1}\right)$ and $\mathcal{G}_{2}=\left(\mathcal{P}_{2}, \mathcal{Q}_{2}\right)$ be two PFGs. For any vertex $\left(x_{1}, x_{2}\right) \in V_{1} \times V_{2}$,

$$
\begin{aligned}
\left(d_{\mu}\right)_{\mathcal{G}_{1}\left[\mathcal{G}_{2}\right]}\left(x_{1}, x_{2}\right)= & \sum_{\left(x_{1}, x_{2}\right)\left(y_{1}, y_{2}\right) \in E_{1} \circ E_{2}}\left(\mu_{\mathcal{Q}_{1}} \circ \mu_{\mathcal{Q}_{2}}\right)\left(\left(x_{1}, x_{2}\right)\left(y_{1}, y_{2}\right)\right) \\
= & \sum_{x_{1}=y_{1}, x_{2} y_{2} \in E_{2}} \mu_{\mathcal{P}_{1}}\left(x_{1}\right) \wedge \mu_{\mathcal{Q}_{2}}\left(x_{2} y_{2}\right)+\sum_{x_{2}=y_{2}, x_{1} y_{1} \in E_{1}} \mu_{\mathcal{P}_{2}}\left(x_{2}\right) \wedge \mu_{\mathcal{Q}_{1}}\left(x_{1} y_{1}\right) \\
& +\sum_{x_{2} \neq y_{2}, x_{1} y_{1} \in E_{1}} \mu_{\mathcal{P}_{2}}\left(y_{2}\right) \wedge \mu_{\mathcal{P}_{2}}\left(x_{2}\right) \wedge \mu_{\mathcal{Q}_{1}}\left(x_{1} y_{1}\right), \\
\left(d_{v}\right)_{\mathcal{G}_{1}\left[\mathcal{G}_{2}\right]}\left(x_{1}, x_{2}\right)= & \sum_{\left(x_{1}, x_{2}\right)\left(y_{1}, y_{2}\right) \in E_{1} \circ E_{2}}\left(v_{\mathcal{Q}_{1}} \circ v_{\mathcal{Q}_{2}}\right)\left(\left(x_{1}, x_{2}\right)\left(y_{1}, y_{2}\right)\right) \\
= & \sum_{1} \sum_{y_{1}, x_{2} y_{2} \in E_{2}} v_{\mathcal{P}_{1}}\left(x_{1}\right) \vee v_{\mathcal{Q}_{2}}\left(x_{2} y_{2}\right)+\sum_{x_{2}=y_{2}, x_{1} y_{1} \in E_{1}} v_{\mathcal{P}_{2}}\left(x_{2}\right) \vee v_{\mathcal{Q}_{1}}\left(x_{1} y_{1}\right) \\
& +\sum_{x_{2} \neq y_{2}, x_{1} y_{1} \in E_{1}} v_{\mathcal{P}_{2}}\left(y_{2}\right) \vee v_{\mathcal{P}_{2}}\left(x_{2}\right) \vee v_{\mathcal{Q}_{1}}\left(x_{1} y_{1}\right) .
\end{aligned}
$$

Theorem 9. Let $\mathcal{G}_{1}=\left(\mathcal{P}_{1}, \mathcal{Q}_{1}\right)$ and $\mathcal{G}_{2}=\left(\mathcal{P}_{2}, \mathcal{Q}_{2}\right)$ be two PFGs. If $\mu_{\mathcal{P}_{1}} \geq \mu_{\mathcal{Q}_{2}}, v_{\mathcal{P}_{1}} \leq v_{\mathcal{Q}_{2}}$ and $\mu_{\mathcal{P}_{2}} \geq \mu_{\mathcal{Q}_{1}}, v_{\mathcal{P}_{2}} \leq v_{\mathcal{Q}_{1}}$. Then, $d_{\mathcal{G}_{1}\left[\mathcal{G}_{2}\right]}\left(x_{1}, x_{2}\right)=p_{2} d_{\mathcal{G}_{1}}\left(x_{1}\right)+d_{\mathcal{G}_{2}}\left(x_{2}\right)$ for all $\left(x_{1}, x_{2}\right) \in V_{1} \times V_{2}$.

Proof. For any vertex $\left(x_{1}, x_{2}\right) \in V_{1} \times V_{2}$,

$$
\begin{aligned}
\left(d_{\mu}\right)_{\mathcal{G}_{1}\left[\mathcal{G}_{2}\right]}\left(x_{1}, x_{2}\right)= & \sum_{\left(x_{1}, x_{2}\right)\left(y_{1}, y_{2}\right) \in E_{1} \circ E_{2}}\left(\mu_{\mathcal{Q}_{1}} \circ \mu_{\mathcal{Q}_{2}}\right)\left(\left(x_{1}, x_{2}\right)\left(y_{1}, y_{2}\right)\right) \\
= & \sum_{x_{1}=y_{1}, x_{2} y_{2 \in E_{2}}} \mu_{\mathcal{P}_{1}}\left(x_{1}\right) \wedge \mu_{\mathcal{Q}_{2}}\left(x_{2} y_{2}\right)+\sum_{x_{2}=y_{2}, x_{1} y_{1} \in E_{1}} \mu_{\mathcal{P}_{2}}\left(x_{2}\right) \wedge \mu_{\mathcal{Q}_{1}}\left(x_{1} y_{1}\right) \\
& +\sum_{x_{2} \neq y_{2}, x_{1} y_{1} \in E_{1}} \mu_{\mathcal{P}_{2}}\left(y_{2}\right) \wedge \mu_{\mathcal{P}_{2}}\left(x_{2}\right) \wedge \mu_{\mathcal{Q}_{1}}\left(x_{1} y_{1}\right) \\
= & \sum_{x_{2} y_{2 \in E_{2}}} \mu_{\mathcal{Q}_{2}}\left(x_{2} y_{2}\right)+\sum_{x_{1} y_{1} \in E_{1}} \mu_{\mathcal{Q}_{1}}\left(x_{1} y_{1}\right)+\sum_{x_{1} y_{1} \in E_{1}} \mu_{\mathcal{Q}_{1}}\left(x_{1} y_{1}\right) \\
& \left(\text { Since } \mu_{\mathcal{P}_{1}} \geq \mu_{\mathcal{Q}_{2}} \text { and } \mu_{\mathcal{P}_{2}} \geq \mu_{\left.\mathcal{Q}_{1}\right)}\right) \\
= & \left(d_{\mu}\right)_{\mathcal{G}_{2}}\left(x_{2}\right)+p_{2}\left(d_{\mu}\right)_{\mathcal{G}_{1}}\left(x_{1}\right) .
\end{aligned}
$$

Analogously, we can show that $\left(d_{v}\right)_{\mathcal{G}_{1}\left[\mathcal{G}_{2}\right]}\left(x_{1}, x_{2}\right)=\left(d_{v}\right)_{\mathcal{G}_{2}}\left(x_{2}\right)+p_{2}\left(d_{v}\right)_{\mathcal{G}_{1}}\left(x_{1}\right)$. Hence, $d_{\mathcal{G}_{1}\left[\mathcal{G}_{2}\right]}\left(x_{1}, x_{2}\right)=d_{\mathcal{G}_{2}}\left(x_{2}\right)+p_{2} d_{\mathcal{G}_{1}}\left(x_{1}\right)$. 
Definition 18. Let $\mathcal{G}_{1}=\left(\mathcal{P}_{1}, \mathcal{Q}_{1}\right)$ and $\mathcal{G}_{2}=\left(\mathcal{P}_{2}, \mathcal{Q}_{2}\right)$ be two PFGs. For any vertex $\left(x_{1}, x_{2}\right) \in V_{1} \times V_{2}$,

$$
\begin{aligned}
\left(t d_{\mu}\right)_{\mathcal{G}_{1}\left[\mathcal{G}_{2}\right]}\left(x_{1}, x_{2}\right)= & \sum_{\left(x_{1}, x_{2}\right)\left(y_{1}, y_{2}\right) \in E_{1} \circ E_{2}}\left(\mu_{\mathcal{Q}_{1}} \circ \mu_{\mathcal{Q}_{2}}\right)\left(\left(x_{1}, x_{2}\right)\left(y_{1}, y_{2}\right)\right)+\left(\mu_{\mathcal{P}_{1}} \circ \mu_{\mathcal{P}_{2}}\right)\left(x_{1}, x_{2}\right) \\
= & \sum_{x_{1}=y_{1}, x_{2} y_{2} \in E_{2}} \mu_{\mathcal{P}_{1}}\left(x_{1}\right) \wedge \mu_{\mathcal{Q}_{2}}\left(x_{2} y_{2}\right)+\sum_{x_{2}=y_{2}, x_{1} y_{1} \in E_{1}} \mu_{\mathcal{P}_{2}}\left(x_{2}\right) \wedge \mu_{\mathcal{Q}_{1}}\left(x_{1} y_{1}\right) \\
& +\sum_{x_{2} \neq y_{2}, x_{1} y_{1} \in E_{1}} \mu_{\mathcal{P}_{2}}\left(y_{2}\right) \wedge \mu_{\mathcal{P}_{2}}\left(x_{2}\right) \wedge \mu_{\mathcal{Q}_{1}}\left(x_{1} y_{1}\right)+\mu_{\mathcal{P}_{1}}\left(x_{1}\right) \wedge \mu_{\mathcal{P}_{2}}\left(x_{2}\right), \\
\left(t d_{v}\right)_{\mathcal{G}_{1}\left[\mathcal{G}_{2}\right]}\left(x_{1}, x_{2}\right)= & \sum_{\left(x_{1}, x_{2}\right)\left(y_{1}, y_{2}\right) \in E_{1} \circ E_{2}}\left(v_{\mathcal{Q}_{1}} \circ v_{\mathcal{Q}_{2}}\right)\left(\left(x_{1}, x_{2}\right)\left(y_{1}, y_{2}\right)\right)+\left(v_{\mathcal{P}_{1}} \circ v_{\mathcal{P}_{2}}\right)\left(x_{1}, x_{2}\right) \\
= & \sum_{x_{1}=y_{1}, x_{2} y_{2} \in E_{2}} v_{\mathcal{P}_{1}}\left(x_{1}\right) \vee v_{\mathcal{Q}_{2}}\left(x_{2} y_{2}\right)+\sum_{x_{2}=y_{2}, x_{1} y_{1} \in E_{1}} v_{\mathcal{P}_{2}}\left(x_{2}\right) \vee v_{\mathcal{Q}_{1}}\left(x_{1} y_{1}\right) \\
& +\sum_{x_{2} \neq y_{2}, x_{1} y_{1} \in E_{1}} v_{\mathcal{P}_{2}}\left(y_{2}\right) \vee v_{\mathcal{P}_{2}}\left(x_{2}\right) \vee v_{\mathcal{Q}_{1}}\left(x_{1} y_{1}\right)+v_{\mathcal{P}_{1}}\left(x_{1}\right) \vee v_{\mathcal{P}_{2}}\left(x_{2}\right) .
\end{aligned}
$$

Theorem 10. Let $\mathcal{G}_{1}=\left(\mathcal{P}_{1}, \mathcal{Q}_{1}\right)$ and $\mathcal{G}_{2}=\left(\mathcal{P}_{2}, \mathcal{Q}_{2}\right)$ be two PFGs. If

(i) $\mu_{\mathcal{P}_{1}} \geq \mu_{\mathcal{Q}_{2}}$ and $\mu_{\mathcal{P}_{2}} \geq \mu_{\mathcal{Q}_{1}}$, then $\left(t d_{\mu}\right)_{\mathcal{G}_{1}\left[\mathcal{G}_{2}\right]}\left(x_{1}, x_{2}\right)=\left(t d_{\mu}\right)_{\mathcal{G}_{2}}\left(x_{2}\right)+p_{2}\left(t d_{\mu}\right)_{\mathcal{G}_{1}}\left(x_{1}\right)-\left(p_{2}-\right.$ 1) $\mu_{\mathcal{P}_{1}}\left(x_{1}\right)-\mu_{\mathcal{P}_{1}}\left(x_{1}\right) \vee \mu_{\mathcal{P}_{2}}\left(x_{2}\right)$;

(ii) $v_{\mathcal{P}_{1}} \leq v_{\mathcal{Q}_{2}}$ and $v_{\mathcal{P}_{2}} \leq v_{\mathcal{Q}_{1}}$, then $\left(t d_{v}\right)_{\mathcal{G}_{1}\left[\mathcal{G}_{2}\right]}\left(x_{1}, x_{2}\right)=\left(t d_{v}\right)_{\mathcal{G}_{2}}\left(x_{2}\right)+p_{2}\left(t d_{v}\right)_{\mathcal{G}_{1}}\left(x_{1}\right)-\left(p_{2}-\right.$ 1) $v_{\mathcal{P}_{1}}\left(x_{1}\right)-v_{\mathcal{P}_{1}}\left(x_{1}\right) \wedge v_{\mathcal{P}_{2}}\left(x_{2}\right)$

for all $\left(x_{1}, x_{2}\right) \in V_{1} \times V_{2}$.

Proof. For any vertex $\left(x_{1}, x_{2}\right) \in V_{1} \times V_{2}$,

(i) If $\mu_{\mathcal{P}_{1}} \geq \mu_{\mathcal{Q}_{2}}, \mu_{\mathcal{P}_{2}} \geq \mu_{\mathcal{Q}_{1}}$

$$
\begin{aligned}
\left(t d_{\mu}\right)_{\mathcal{G}_{1}\left[\mathcal{G}_{2}\right]}\left(x_{1}, x_{2}\right)= & \sum_{x_{1}=y_{1}, x_{2} y_{2} \in E_{2}} \mu_{\mathcal{P}_{1}}\left(x_{1}\right) \wedge \mu_{\mathcal{Q}_{2}}\left(x_{2} y_{2}\right)+\sum_{x_{2}=y_{2}, x_{1} y_{1} \in E_{1}} \mu_{\mathcal{P}_{2}}\left(x_{2}\right) \wedge \mu_{\mathcal{Q}_{1}}\left(x_{1} y_{1}\right) \\
& +\sum_{x_{2} \neq y_{2}, x_{1} y_{1} \in E_{1}} \mu_{\mathcal{P}_{2}}\left(y_{2}\right) \wedge \mu_{\mathcal{P}_{2}}\left(x_{2}\right) \wedge \mu_{\mathcal{Q}_{1}}\left(x_{1} y_{1}\right)+\mu_{\mathcal{P}_{1}}\left(x_{1}\right) \wedge \mu_{\mathcal{P}_{2}}\left(x_{2}\right) \\
= & \sum_{x_{2} y_{2} \in E_{2}} \mu_{\mathcal{Q}_{2}}\left(x_{2} y_{2}\right)+\sum_{x_{1} y_{1} \in E_{1}} \mu_{\mathcal{Q}_{1}}\left(x_{1} y_{1}\right)+\sum_{x_{1} y_{1} \in E_{1}} \mu_{\mathcal{Q}_{1}}\left(x_{1} y_{1}\right) \\
& +\mu_{\mathcal{P}_{1}}\left(x_{1}\right)+\mu_{\mathcal{P}_{2}}\left(x_{2}\right)-\mu_{\mathcal{P}_{1}}\left(x_{1}\right) \vee \mu_{\mathcal{P}_{2}}\left(x_{2}\right) \\
= & \left(t d_{\mu}\right)_{\mathcal{G}_{2}}\left(x_{2}\right)+p_{2}\left(t d_{\mu}\right)_{\mathcal{G}_{1}}\left(x_{1}\right)-\left(p_{2}-1\right) \mu_{\mathcal{P}_{1}}\left(x_{1}\right) \\
& -\mu_{\mathcal{P}_{1}}\left(x_{1}\right) \vee \mu_{\mathcal{P}_{2}}\left(x_{2}\right) .
\end{aligned}
$$

Analogously, we can prove (ii).

Example 6. Consider two PFGs $\mathcal{G}_{1}$ and $\mathcal{G}_{2}$ as in Example 2, where $\mu_{\mathcal{P}_{1}} \geq \mu_{\mathcal{Q}_{2}}, v_{\mathcal{P}_{1}} \leq v_{\mathcal{Q}_{2}}$ and $\mu_{\mathcal{P}_{2}} \geq \mu_{\mathcal{Q}_{1}}$, $v_{\mathcal{P}_{2}} \leq v_{\mathcal{Q}_{1}}$ and their lexicographic product $\mathcal{G}_{1} \circ \mathcal{G}_{2}$ is shown in Figure 8.

Then, by Theorem 9, we must have

$$
\begin{array}{r}
\left(d_{\mu}\right)_{\mathcal{G}_{1} \circ \mathcal{G}_{2}}(m, p)=p_{2}\left(d_{\mu}\right)_{\mathcal{G}_{1}}(m)+\left(d_{\mu}\right)_{\mathcal{G}_{2}}(p)=2.7 \\
\left(d_{v}\right)_{\mathcal{G}_{1} \circ \mathcal{G}_{2}}(m, p)=p_{2}\left(d_{v}\right)_{\mathcal{G}_{1}}(m)+\left(d_{v}\right)_{\mathcal{G}_{2}}(p)=3.6
\end{array}
$$

Therefore, $d_{\mathcal{G}_{1} \circ \mathcal{G}_{2}}(m, p)=(2.7,3.6)$.

In addition, by Theorem 10, we must have

$$
\begin{aligned}
& \left(t d_{\mu}\right)_{\mathcal{G}_{1} \circ \mathcal{G}_{2}}(m, p)=p_{2}\left(t d_{\mu}\right)_{\mathcal{G}_{1}}(m)+\left(t d_{\mu}\right)_{\mathcal{G}_{2}}(p)-\left(p_{2}-1\right) \mu_{\mathcal{P}_{1}}(m)-\mu_{\mathcal{P}_{1}}(m) \vee \mu_{\mathcal{P}_{2}}(p)=3.5, \\
& \left(t d_{v}\right)_{\mathcal{G}_{1} \circ \mathcal{G}_{2}}(m, p)=p_{2}\left(t d_{v}\right)_{\mathcal{G}_{1}}(m)+\left(t d_{v}\right)_{\mathcal{G}_{2}}(p)-\left(p_{2}-1\right) v_{\mathcal{P}_{1}}(m)-v_{\mathcal{P}_{1}}(m) \wedge v_{\mathcal{P}_{2}}(p)=4.2
\end{aligned}
$$

Therefore, $d_{\mathcal{G}_{1} \circ \mathcal{G}_{2}}(m, p)=(3.5,4.2)$.

Similarly, we can find the degree and total degree of all the vertices in $\mathcal{G}_{1} \circ \mathcal{G}_{2}$. 


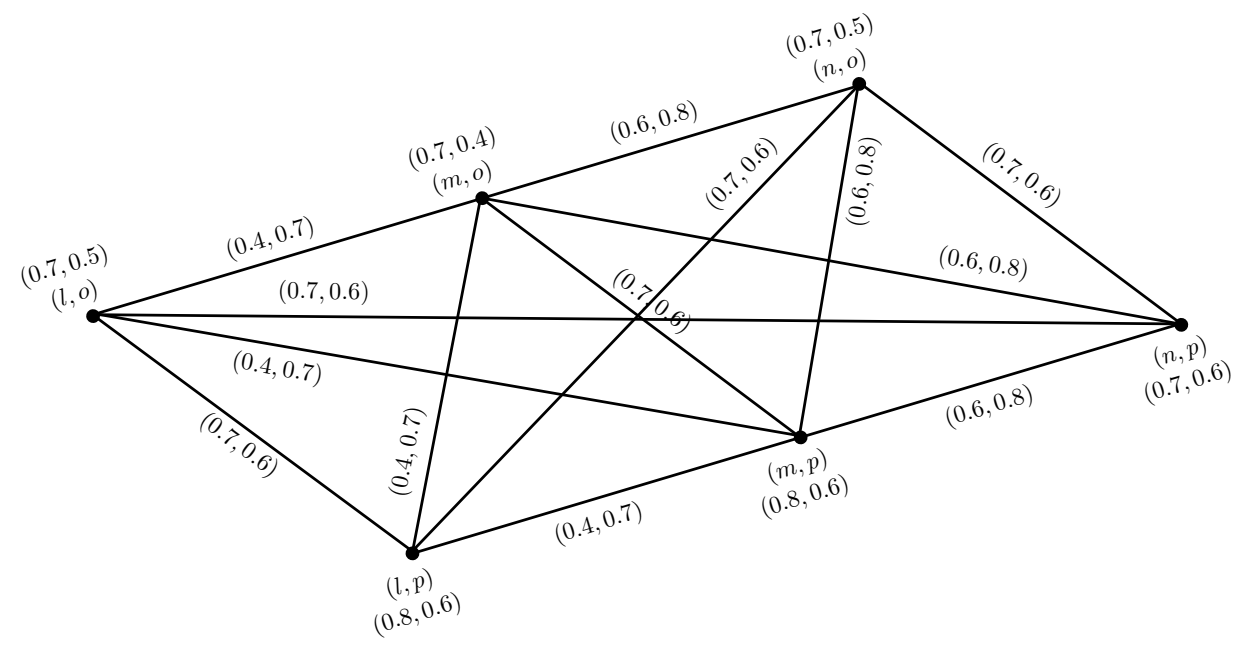

Figure 8. Lexicographic product of two PFGs.

Definition 19. The union $\mathcal{G}_{1} \cup \mathcal{G}_{2}=\left(\mathcal{P}_{1} \cup \mathcal{P}_{2}, \mathcal{Q}_{1} \cup \mathcal{Q}_{2}\right)$ of two PFGs $\mathcal{G}_{1}=\left(\mathcal{P}_{1}, \mathcal{Q}_{1}\right)$ and $\mathcal{G}_{2}=\left(\mathcal{P}_{2}, \mathcal{Q}_{2}\right)$ of the graphs $G_{1}=\left(V_{1}, E_{1}\right)$ and $G_{2}=\left(V_{2}, E_{2}\right)$, respectively, is defined as follows:

(i) $\quad\left(\mu_{\mathcal{P}_{1}} \cup \mu_{\mathcal{P}_{2}}\right)(x)= \begin{cases}\mu_{\mathcal{P}_{1}}(x) & \text { if } x \in V_{1}-V_{2}, \\ \mu_{\mathcal{P}_{2}}(x) & \text { if } x \in V_{2}-V_{1}, \\ \mu_{\mathcal{P}_{1}}(x) \vee \mu_{\mathcal{P}_{2}}(x) & \text { if } x \in V_{1} \cap V_{2},\end{cases}$

(ii) $\left(v_{\mathcal{P}_{1}} \cup v_{\mathcal{P}_{2}}\right)(x)= \begin{cases}v_{\mathcal{P}_{1}}(x) & \text { if } x \in V_{1}-V_{2}, \\ v_{\mathcal{P}_{2}}(x) & \text { if } x \in V_{2}-V_{1}, \\ v_{\mathcal{P}_{1}}(x) \wedge v_{\mathcal{P}_{2}}(x) & \text { if } x \in V_{1} \cap V_{2},\end{cases}$

(iii) $\left(\mu_{\mathcal{Q}_{1}} \cup \mu_{\mathcal{Q}_{2}}\right)(x y)= \begin{cases}\mu_{\mathcal{Q}_{1}}(x y) & \text { if } x y \in E_{1}-E_{2}, \\ \mu_{\mathcal{Q}_{2}}(x y) & \text { if } x y \in E_{2}-E_{1}, \\ \mu_{\mathcal{Q}_{1}}(x y) \vee \mu_{\mathcal{Q}_{2}}(x y) & \text { if } x y \in E_{1} \cap E_{2},\end{cases}$

(iv) $\left(v_{\mathcal{Q}_{1}} \cup v_{\mathcal{Q}_{2}}\right)(x y)= \begin{cases}v_{\mathcal{Q}_{1}}(x y) & \text { if } x y \in E_{1}-E_{2}, \\ v_{\mathcal{Q}_{2}}(x y) & \text { if } x y \in E_{2}-E_{1}, \\ v_{\mathcal{Q}_{1}}(x y) \wedge v_{\mathcal{Q}_{2}}(x y) & \text { if } x y \in E_{1} \cap E_{2} .\end{cases}$

Theorem 11. The union $\mathcal{G}_{1} \cup \mathcal{G}_{2}$ of $\mathcal{G}_{1}$ and $\mathcal{G}_{2}$ is a PFG of $G_{1} \cup G_{2}$ if and only if $\mathcal{G}_{1}$ and $\mathcal{G}_{2}$ are PFGs of $G_{1}$ and $G_{2}$, respectively, where $\mathcal{P}_{1}, \mathcal{P}_{2}, \mathcal{Q}_{1}$ and $\mathcal{Q}_{2}$ are the Pythagorean fuzzy subsets of $V_{1}, V_{2}, E_{1}$ and $E_{2}$, respectively and $V_{1} \cap V_{2}=\varnothing$.

Definition 20. Let $\mathcal{G}_{1}=\left(\mathcal{P}_{1}, \mathcal{Q}_{1}\right)$ and $\mathcal{G}_{2}=\left(\mathcal{P}_{2}, \mathcal{Q}_{2}\right)$ be two PFGs. For any vertex $x \in V_{1} \cup V_{2}$, there are three cases to consider.

Case 1: Either $x \in V_{1}-V_{2}$ or $x \in V_{2}-V_{1}$. Then, no edge incident at $x$ lies in $E_{1} \cap E_{2}$. Thus, for $x \in V_{1}-V_{2}$,

$$
\begin{aligned}
\left(d_{\mu}\right)_{\mathcal{G}_{1} \cup \mathcal{G}_{2}}(x)= & \sum_{x y \in E_{1}} \mu_{\mathcal{Q}_{1}}(x y)=\left(d_{\mu}\right)_{\mathcal{G}_{1}}(x),\left(d_{v}\right)_{\mathcal{G}_{1} \cup \mathcal{G}_{2}}(x)=\sum_{x y \in E_{1}} v_{\mathcal{Q}_{1}}(x y)=\left(d_{v}\right)_{\mathcal{G}_{1}}(x), \\
& \left(t d_{\mu}\right)_{\mathcal{G}_{1} \cup \mathcal{G}_{2}}(x)=\left(t d_{\mu}\right)_{\mathcal{G}_{1}}(x),\left(t d_{v}\right)_{\mathcal{G}_{1} \cup \mathcal{G}_{2}}(x)=\left(t d_{v}\right)_{\mathcal{G}_{1}}(x) .
\end{aligned}
$$

For $x \in V_{2}-V_{1}$,

$$
\begin{gathered}
\left(d_{\mu}\right)_{\mathcal{G}_{1} \cup \mathcal{G}_{2}}(x)=\sum_{x y \in E_{2}} \mu_{\mathcal{Q}_{2}}(x y)=\left(d_{\mu}\right)_{\mathcal{G}_{2}}(x),\left(d_{v}\right)_{\mathcal{G}_{1} \cup \mathcal{G}_{2}}(x)=\sum_{x y \in E_{2}} v_{\mathcal{Q}_{2}}(x y)=\left(d_{v}\right)_{\mathcal{G}_{2}}(x) . \\
\left(t d_{\mu}\right)_{\mathcal{G}_{1} \cup \mathcal{G}_{2}}(x)=\left(t d_{\mu}\right)_{\mathcal{G}_{2}}(x),\left(t d_{v}\right)_{\mathcal{G}_{1} \cup \mathcal{G}_{2}}(x)=\left(t d_{v}\right)_{\mathcal{G}_{2}}(x)
\end{gathered}
$$


Case 2: $x \in V_{1} \cap V_{2}$ but no edge incident at $x$ lies in $E_{1} \cap E_{2}$. Then, any edge incident at $x$ is either in $E_{1}-E_{2}$ or in $E_{2}-E_{1}$.

$$
\begin{aligned}
\left(d_{\mu}\right)_{\mathcal{G}_{1} \cup \mathcal{G}_{2}}(x) & =\sum_{x y \in E_{1} \cup E_{2}}\left(\mu_{\mathcal{Q}_{1}} \cup \mu_{\mathcal{Q}_{2}}\right)(x y) \\
& =\sum_{x y \in E_{1}} \mu_{\mathcal{Q}_{1}}(x y)+\sum_{x y \in E_{2}} \mu_{\mathcal{Q}_{2}}(x y) \\
& =\left(d_{\mu}\right)_{\mathcal{G}_{1}}(x)+\left(d_{\mu}\right)_{\mathcal{G}_{2}}(x) .
\end{aligned}
$$

Similarly, $\left(d_{v}\right)_{\mathcal{G}_{1} \cup \mathcal{G}_{2}}(x)=\left(d_{v}\right)_{\mathcal{G}_{1}}(x)+\left(d_{v}\right)_{\mathcal{G}_{2}}(x)$,

$$
\begin{aligned}
\left(t d_{\mu}\right)_{\mathcal{G}_{1} \cup \mathcal{G}_{2}}(x) & =\sum_{x y \in E_{1} \cup E_{2}}\left(\mu_{\mathcal{Q}_{1}} \cup \mu_{\mathcal{Q}_{2}}\right)(x y)+\mu_{\mathcal{P}_{1}}(x) \vee \mu_{\mathcal{P}_{2}}(x) \\
& =\left(d_{\mu}\right)_{\mathcal{G}_{1}}(x)+\left(d_{\mu}\right)_{\mathcal{G}_{2}}(x)+\mu_{\mathcal{P}_{1}}(x) \vee \mu_{\mathcal{P}_{2}}(x) \\
& =\left(t d_{\mu}\right)_{\mathcal{G}_{1}}(x)+\left(t d_{\mu}\right)_{\mathcal{G}_{2}}(x)-\mu_{\mathcal{P}_{1}}(x) \wedge \mu_{\mathcal{P}_{2}}(x) .
\end{aligned}
$$

Similarly, $\left(t d_{v}\right)_{\mathcal{G}_{1} \cup \mathcal{G}_{2}}(x)=\left(t d_{v}\right)_{\mathcal{G}_{1}}(x)+\left(t d_{v}\right)_{\mathcal{G}_{2}}(x)-v_{\mathcal{P}_{1}}(x) \vee v_{\mathcal{P}_{2}}(x)$.

Case 3: $x \in V_{1} \cap V_{2}$ and some edges incident at $x$ are in $E_{1} \cap E_{2}$.

$$
\begin{aligned}
\left(d_{\mu}\right)_{\mathcal{G}_{1} \cup \mathcal{G}_{2}}(x)= & \sum_{x y \in E_{1} \cup E_{2}}\left(\mu_{\mathcal{Q}_{1}} \cup \mu_{\mathcal{Q}_{2}}\right)(x y) \\
& \sum_{x y \in E_{1}-E_{2}} \mu_{\mathcal{Q}_{1}}(x y)+\sum_{x y \in E_{2}-E_{1}} \mu_{\mathcal{Q}_{2}}(x y)+\sum_{x y \in E_{1} \cap E_{2}} \mu_{\mathcal{Q}_{1}}(x y) \vee \mu_{\mathcal{Q}_{2}}(x y) \\
= & \left(\sum_{x y \in E_{1}-E_{2}} \mu_{\mathcal{Q}_{1}}(x y)+\sum_{x y \in E_{2}-E_{1}} \mu_{\mathcal{Q}_{2}}(x y)+\sum_{x y \in E_{1} \cap E_{2}} \mu_{\mathcal{Q}_{1}}(x y) \vee \mu_{\mathcal{Q}_{2}}(x y)\right. \\
& \left.+\sum_{x y \in E_{1} \cap E_{2}} \mu_{\mathcal{Q}_{1}}(x y) \wedge \mu_{\mathcal{Q}_{2}}(x y)\right)-\sum_{x y \in E_{1} \cap E_{2}} \mu_{\mathcal{Q}_{1}}(x y) \wedge \mu_{\mathcal{Q}_{2}}(x y) \\
= & \sum_{x y \in E_{1}} \mu_{\mathcal{Q}_{1}}(x y)+\sum_{x y \in E_{2}} \mu_{\mathcal{Q}_{2}}(x y)-\sum_{x y \in E_{1} \cap E_{2}} \mu_{\mathcal{Q}_{1}}(x y) \wedge \mu_{\mathcal{Q}_{2}}(x y) \\
= & \left(d_{\mu}\right)_{\mathcal{G}_{1}}(x)+\left(d_{\mu}\right) \mathcal{G}_{\mathcal{G}_{2}}(x)-\sum_{x y \in E_{1} \cap E_{2}} \mu_{\mathcal{Q}_{1}}(x y) \wedge \mu_{\mathcal{Q}_{2}}(x y) .
\end{aligned}
$$

Similarly, $\left(d_{v}\right)_{\mathcal{G}_{1} \cup \mathcal{G}_{2}}(x)=\left(d_{v}\right)_{\mathcal{G}_{1}}(x)+\left(d_{v}\right)_{\mathcal{G}_{2}}(x)-\sum_{x y \in E_{1} \cap E_{2}} v_{\mathcal{Q}_{1}}(x y) \vee v_{\mathcal{Q}_{2}}(x y)$. In addition,

$$
\begin{aligned}
& \left(t d_{\mu}\right)_{\mathcal{G}_{1} \cup \mathcal{G}_{2}}(x)=\left(t d_{\mu}\right)_{\mathcal{G}_{1}}(x)+\left(t d_{\mu}\right)_{\mathcal{G}_{2}}(x)-\sum_{x y \in E_{1} \cap E_{2}} \mu_{\mathcal{Q}_{1}}(x y) \wedge \mu_{\mathcal{Q}_{2}}(x y)-\mu_{\mathcal{P}_{1}}(x) \wedge \mu_{\mathcal{P}_{2}}(x), \\
& \left(t d_{v}\right)_{\mathcal{G}_{1} \cup \mathcal{G}_{2}}(x)=\left(t d_{v}\right)_{\mathcal{G}_{1}}(x)+\left(t d_{v}\right)_{\mathcal{G}_{2}}(x)-\sum_{x y \in E_{1} \cap E_{2}} v_{\mathcal{Q}_{1}}(x y) \vee v_{\mathcal{Q}_{2}}(x y)-\mu_{\mathcal{P}_{1}}(x) \vee v_{\mathcal{P}_{2}}(x)
\end{aligned}
$$

Example 7. Consider two PFGs $\mathcal{G}_{1}=\left(\mathcal{P}_{1}, \mathcal{Q}_{1}\right)$ and $\mathcal{G}_{2}=\left(\mathcal{P}_{2}, \mathcal{Q}_{2}\right)$ on $V_{1}=\{j, k, l, m\}$ and $V_{2}=\{j, k, l\}$, respectively, as shown in Figure 9.

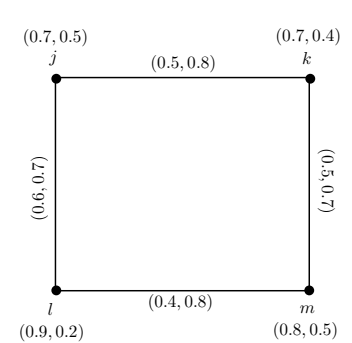

$\mathcal{G}_{1}$

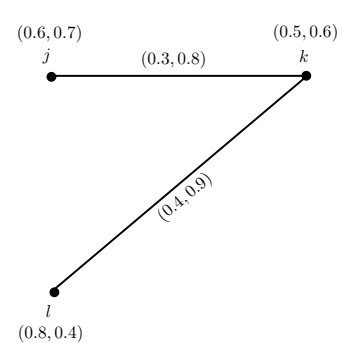

$\mathcal{G}_{2}$

Figure 9. PFGs. 
In addition, their union $\mathcal{G}_{1} \cup \mathcal{G}_{2}$ is shown in Figure 10.

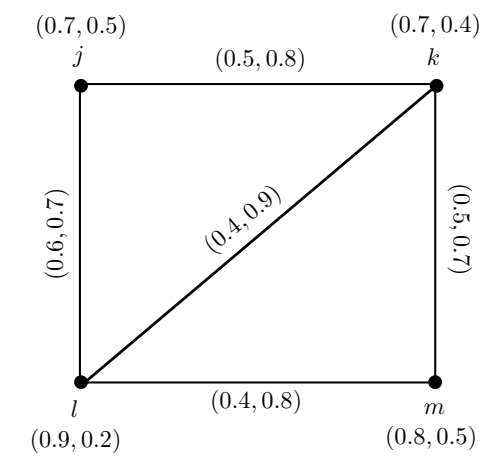

Figure 10. Union of two PFGs.

Since $m \in V_{1} \backslash V_{2}$, thus,

$$
\left(d_{\mu}\right)_{\mathcal{G}_{1} \cup \mathcal{G}_{2}}(m)=\left(d_{\mu}\right)_{\mathcal{G}_{1}}(m)=0.9,\left(d_{\nu}\right)_{\mathcal{G}_{1} \cup \mathcal{G}_{2}}(m)=\left(d_{\nu}\right)_{\mathcal{G}_{1}}(m)=1.5
$$

Therefore, $d_{\mathcal{G}_{1} \cup \mathcal{G}_{2}}(m)=d_{\mathcal{G}_{1}}(m)=(0.9,1.5)$.

$$
\left(t d_{\mu}\right)_{\mathcal{G}_{1} \cup \mathcal{G}_{2}}(m)=\left(t d_{\mu}\right)_{\mathcal{G}_{1}}(m)=1.7,\left(t d_{v}\right)_{\mathcal{G}_{1} \cup \mathcal{G}_{2}}(m)=\left(t d_{\nu}\right)_{\mathcal{G}_{1}}(m)=2.0
$$

Therefore, $t d_{\mathcal{G}_{1} \cup \mathcal{G}_{2}}(m)=t d_{\mathcal{G}_{1}}(m)=(1.7,2.0)$.

Since $l \in V_{1} \cap V_{2}$ but no edge incident at l lies in $E_{1} \cap E_{2}$,

$$
\begin{aligned}
& \left(d_{\mu}\right)_{\mathcal{G}_{1} \cup \mathcal{G}_{2}}(l)=\left(d_{\mu}\right)_{\mathcal{G}_{1}}(l)+\left(d_{\mu}\right)_{\mathcal{G}_{2}}(l)=1.4, \\
& \left(d_{v}\right)_{\mathcal{G}_{1} \cup \mathcal{G}_{2}}(l)=\left(d_{v}\right)_{\mathcal{G}_{1}}(l)+\left(d_{v}\right)_{\mathcal{G}_{2}}(l)=2.4 .
\end{aligned}
$$

Therefore, $d_{\mathcal{G}_{1} \cup \mathcal{G}_{2}}(l)=d_{\mathcal{G}_{1}}(l)+d_{\mathcal{G}_{2}}(l)=(1.4,2.4)$,

$$
\begin{aligned}
& \left(t d_{\mu}\right)_{\mathcal{G}_{1} \cup \mathcal{G}_{2}}(l)=\left(t d_{\mu}\right)_{\mathcal{G}_{1}}(l)+\left(t d_{\mu}\right)_{\mathcal{G}_{2}}(l)-\mu_{\mathcal{P}_{1}}(l) \wedge \mu_{\mathcal{P}_{2}}(l)=2.3 \\
& \left(t d_{v}\right)_{\mathcal{G}_{1} \cup \mathcal{G}_{2}}(l)=\left(t d_{v}\right)_{\mathcal{G}_{1}}(l)+\left(t d_{v}\right)_{\mathcal{G}_{2}}(l)-v_{\mathcal{P}_{1}}(l) \vee v_{\mathcal{P}_{2}}(l)=2.6 .
\end{aligned}
$$

Therefore, $t d_{\mathcal{G}_{1} \cup \mathcal{G}_{2}}(l)=(2.3,2.6)$.

Since $k \in V_{1} \cap V_{2}$ and $j k \in E_{1} \cap E_{2}$, thus,

$$
\begin{aligned}
& \left(d_{\mu}\right)_{\mathcal{G}_{1} \cup \mathcal{G}_{2}}(k)=\left(d_{\mu}\right)_{\mathcal{G}_{1}}(k)+\left(d_{\mu}\right)_{\mathcal{G}_{2}}(k)-\mu_{\mathcal{Q}_{1}}(j k) \wedge \mu_{\mathcal{Q}_{2}}(j k)=1.4 \\
& \left(d_{v}\right)_{\mathcal{G}_{1} \cup \mathcal{G}_{2}}(k)=\left(d_{v}\right)_{\mathcal{G}_{1}}(k)+\left(d_{v}\right)_{\mathcal{G}_{2}}(k)-v_{\mathcal{Q}_{1}}(j k) \vee v_{\mathcal{Q}_{2}}(j k)=2.4
\end{aligned}
$$

Therefore, $d_{\mathcal{G}_{1} \cup \mathcal{G}_{2}}(k)=(1.4,2.4)$ :

$$
\begin{aligned}
\left(t d_{\mu}\right)_{\mathcal{G}_{1} \cup \mathcal{G}_{2}}(k) & =\left(t d_{\mu}\right)_{\mathcal{G}_{1}}(k)+\left(t d_{\mu}\right)_{\mathcal{G}_{2}}(k)-\mu_{\mathcal{Q}_{1}}(j k) \wedge \mu_{\mathcal{Q}_{2}}(j k)-\mu_{\mathcal{P}_{1}}(k) \wedge \mu_{\mathcal{P}_{2}}(k) \\
& =1.7+1.2-0.3-0.5=2.1 \\
\left(t d_{v}\right)_{\mathcal{G}_{1} \cup \mathcal{G}_{2}}(k) & =\left(t d_{v}\right)_{\mathcal{G}_{1}}(k)+\left(t d_{v}\right)_{\mathcal{G}_{2}}(k)-v_{\mathcal{Q}_{1}}(j k) \vee v_{\mathcal{Q}_{2}}(j k)-v_{\mathcal{P}_{1}}(k) \vee v_{\mathcal{P}_{2}}(k) \\
& =1.9+2.3-0.8-0.6=2.8
\end{aligned}
$$

Therefore, $t d_{\mathcal{G}_{1} \cup \mathcal{G}_{2}}(k)=(2.1,2.8)$.

Definition 21. The ring-sum $\mathcal{G}_{1} \oplus \mathcal{G}_{2}=\left(\mathcal{P}_{1} \oplus \mathcal{P}_{2}, \mathcal{Q}_{1} \oplus \mathcal{Q}_{2}\right)$ of two PFGs $\mathcal{G}_{1}=\left(\mathcal{P}_{1}, \mathcal{Q}_{1}\right)$ and $\mathcal{G}_{2}=\left(\mathcal{P}_{2}, \mathcal{Q}_{2}\right)$ of the graphs $G_{1}=\left(V_{1}, E_{1}\right)$ and $G_{2}=\left(V_{2}, E_{2}\right)$, respectively, is defined as follows:

$$
\left(\mu_{\mathcal{P}_{1}} \oplus \mu_{\mathcal{P}_{2}}\right)(x)=\left(\mu_{\mathcal{P}_{1}} \cup \mu_{\mathcal{P}_{2}}\right)(x),\left(\nu_{\mathcal{P}_{1}} \oplus v_{\mathcal{P}_{2}}\right)(x)=\left(\nu_{\mathcal{P}_{1}} \cup v_{\mathcal{P}_{2}}\right)(x) \quad \text { if } x \in V_{1} \cup V_{2},
$$




$$
\begin{aligned}
& \left(\mu_{\mathcal{Q}_{1}} \oplus \mu_{\mathcal{Q}_{2}}\right)(x y)= \begin{cases}\mu_{\mathcal{Q}_{1}}(x y) & \text { if } x y \in E_{1}-E_{2}, \\
\mu_{\mathcal{Q}_{2}}(x y) & \text { if } x y \in E_{2}-E_{1} \\
0 & \text { if } x y \in E_{1} \cap E_{2} .\end{cases} \\
& \left(v_{\mathcal{Q}_{1}} \oplus v_{\mathcal{Q}_{2}}\right)(x y)= \begin{cases}v_{\mathcal{Q}_{1}}(x y) & \text { if } x y \in E_{1}-E_{2}, \\
v_{\mathcal{Q}_{2}}(x y) & \text { if } x y \in E_{2}-E_{1}, \\
0 & \text { if } x y \in E_{1} \cap E_{2} .\end{cases}
\end{aligned}
$$

Proposition 6. If $\mathcal{G}_{1}=\left(\mathcal{P}_{1}, \mathcal{Q}_{1}\right)$ and $\mathcal{G}_{2}=\left(\mathcal{P}_{2}, \mathcal{Q}_{2}\right)$ are the PFGs, then $\mathcal{G}_{1} \oplus \mathcal{G}_{2}$ is the PFG.

Definition 22. Let $\mathcal{G}_{1}=\left(\mathcal{P}_{1}, \mathcal{Q}_{1}\right)$ and $\mathcal{G}_{2}=\left(\mathcal{P}_{2}, \mathcal{Q}_{2}\right)$ be two PFGs. For any vertex $x \in V_{1} \oplus V_{2}$, there are two cases to consider.

Case 1: If either $x \in V_{1}-V_{2}$ or $x \in V_{2}-V_{1}$.

Case 2: If $x \in V_{1} \cap V_{2}$. Then, any edge incident at $x$ is either in $E_{1}-E_{2}$ or in $E_{2}-E_{1}$.

In both cases:

$$
\begin{aligned}
& \left(d_{\mu}\right)_{\mathcal{G}_{1} \oplus \mathcal{G}_{2}}(x)=\left(d_{\mu}\right)_{\mathcal{G}_{1} \cup \mathcal{G}_{2}}(x),\left(d_{v}\right)_{\mathcal{G}_{1} \oplus \mathcal{G}_{2}}(x)=\left(d_{v}\right)_{\mathcal{G}_{1} \cup \mathcal{G}_{2}}(x), \\
& \left(t d_{\mu}\right)_{\mathcal{G}_{1} \oplus \mathcal{G}_{2}}(x)=\left(t d_{\mu}\right)_{\mathcal{G}_{1} \cup \mathcal{G}_{2}}(x),\left(t d_{v}\right)_{\mathcal{G}_{1} \oplus \mathcal{G}_{2}}(x)=\left(t d_{v}\right)_{\mathcal{G}_{1} \cup \mathcal{G}_{2}}(x) .
\end{aligned}
$$

Definition 23. Let $\mathcal{G}_{1}=\left(\mathcal{P}_{1}, \mathcal{Q}_{1}\right)$ and $\mathcal{G}_{2}=\left(\mathcal{P}_{2}, \mathcal{Q}_{2}\right)$ be two PFGs of $G_{1}=\left(V_{1}, E_{1}\right)$ and $G_{2}=\left(V_{2}, E_{2}\right)$, respectively. The join of $\mathcal{G}_{1}$ and $\mathcal{G}_{2}$, denoted by $\mathcal{G}_{1}+\mathcal{G}_{2}=\left(\mathcal{P}_{1}+\mathcal{P}_{2}, \mathcal{Q}_{1}+\mathcal{Q}_{2}\right)$, is defined as:

(i) $\left\{\begin{array}{l}\left(\mu_{\mathcal{P}_{1}}+\mu_{\mathcal{P}_{2}}\right)(x)=\left(\mu_{\mathcal{P}_{1}} \cup \mu_{\mathcal{P}_{2}}\right)(x) \\ \left(v_{\mathcal{P}_{1}}+v_{\mathcal{P}_{2}}\right)(x)=\left(v_{\mathcal{P}_{1}} \cup v_{\mathcal{P}_{2}}\right)(x) \quad \text { for all } x \in V_{1} \cup V_{2},\end{array}\right.$

(ii) $\left\{\begin{array}{l}\left(\mu_{\mathcal{Q}_{1}}+\mu_{\mathcal{Q}_{2}}\right)(x y)=\left(\mu_{\mathcal{Q}_{1}} \cup \mu_{\mathcal{Q}_{2}}\right)(x y) \\ \left(v_{\mathcal{Q}_{1}}+v_{\mathcal{Q}_{2}}\right)(x y)=\left(v_{\mathcal{Q}_{1}} \cup v_{\mathcal{Q}_{2}}\right)(x y) \quad \text { if } x y \in E_{1} \cup E_{2}\end{array}\right.$

(iii) $\left\{\begin{array}{l}\left(\mu_{\mathcal{Q}_{1}}+\mu_{\mathcal{Q}_{2}}\right)(x y)=\mu_{\mathcal{P}_{1}}(x) \wedge \mu_{\mathcal{P}_{2}}(y) \\ \left(v_{\mathcal{Q}_{1}}+v_{\mathcal{Q}_{2}}\right)(x y)=v_{\mathcal{P}_{1}}(x) \vee v_{\mathcal{P}_{2}}(y) \quad \text { if } x y \in E^{\prime},\end{array}\right.$

where $E^{\prime}$ is the set of all edges joining the vertices of $V_{1}$ and $V_{2}, V_{1} \cap V_{2}=\varnothing$.

Theorem 12. The join $\mathcal{G}_{1}+\mathcal{G}_{2}$ of $\mathcal{G}_{1}$ and $\mathcal{G}_{2}$ is a PFG of $G_{1}+G_{2}$ if and only if $\mathcal{G}_{1}$ and $\mathcal{G}_{2}$ are PFGs of $G_{1}$ and $G_{2}$, respectively, where $\mathcal{P}_{1}, \mathcal{P}_{2}, \mathcal{Q}_{1}$ and $\mathcal{Q}_{2}$ are the Pythagorean fuzzy subsets of $V_{1}, V_{2}, E_{1}$ and $E_{2}$, respectively, and $V_{1} \cap V_{2}=\varnothing$.

Definition 24. Let $\mathcal{G}_{1}=\left(\mathcal{P}_{1}, \mathcal{Q}_{1}\right)$ and $\mathcal{G}_{2}=\left(\mathcal{P}_{2}, \mathcal{Q}_{2}\right)$ be two PFGs. For any vertex $x \in V_{1}+V_{2}$,

$$
\begin{gathered}
\left(d_{\mu}\right)_{\mathcal{G}_{1}+\mathcal{G}_{2}}(x)=\sum_{x y \in E_{1} \cup E_{2}}\left(\mu_{\mathcal{Q}_{1}} \cup \mu_{\mathcal{Q}_{2}}\right)(x y)+\sum_{x y \in E^{\prime}} \mu_{\mathcal{P}_{1}}(x) \wedge \mu_{\mathcal{P}_{2}}(y), \\
\left(d_{v}\right)_{\mathcal{G}_{1}+\mathcal{G}_{2}}(x)=\sum_{x y \in E_{1} \cup E_{2}}\left(v_{\mathcal{Q}_{1}} \cup v_{\mathcal{Q}_{2}}\right)(x y)+\sum_{x y \in E^{\prime}} v_{\mathcal{P}_{1}}(x) \vee v_{\mathcal{P}_{2}}(y) . \\
\left(t d_{\mu}\right)_{\mathcal{G}_{1}+\mathcal{G}_{2}}(x)=\sum_{x y \in E_{1} \cup E_{2}}\left(\mu_{\mathcal{Q}_{1}} \cup \mu_{\mathcal{Q}_{2}}\right)(x y)+\sum_{x y \in E^{\prime}} \mu_{\mathcal{P}_{1}}(x) \wedge \mu_{\mathcal{P}_{2}}(y)+\mu_{\mathcal{P}_{1}}(x) \vee \mu_{\mathcal{P}_{2}}(y), \\
\left(t d_{v}\right)_{\mathcal{G}_{1}+\mathcal{G}_{2}}(x)=\sum_{x y \in E_{1} \cup E_{2}}\left(v_{\mathcal{Q}_{1}} \cup v_{\mathcal{Q}_{2}}\right)(x y)+\sum_{x y \in E^{\prime}} v_{\mathcal{P}_{1}}(x) \vee v_{\mathcal{P}_{2}}(y)+v_{\mathcal{P}_{1}}(x) \wedge v_{\mathcal{P}_{2}}(y) .
\end{gathered}
$$

\section{Applications to Decision-Making}

Decision making is a common activity in daily life, aiming to select the best alternative from a given finite set of alternatives. In actual decision-making problems, decision makers usually rely on 
their intuition and prior expertise to make decisions. Owing to the complexity of decision-making problems, the precondition is to represent the fuzzy and vague information appropriately in the process of decision-making. To express the decision makers' or experts' preferences over the given alternatives (criteria), preference relation is one of the useful techniques by which the ranking of criteria can be obtained. For a set of criteria $X=\left\{x_{1}, x_{2}, \ldots, x_{n}\right\}$, the experts compare each pair of criteria and construct preference relations, respectively. If every element in the preference relations is a PFN, then the concept of the Pythagorean fuzzy preference relation (PFPR) can be put forward as follows:

Definition 25. A PFPR on the set $X=\left\{x_{1}, x_{2}, \ldots, x_{n}\right\}$ is represented by a matrix $R=\left(r_{i j}\right)_{n \times n}$, where $r_{i j}=\left(x_{i} x_{j}, \mu\left(x_{i} x_{j}\right), v\left(x_{i} x_{j}\right)\right)$ for all $i, j=1,2, \ldots, n$. For convenience, let $r_{i j}=\left(\mu_{i j}, v_{i j}\right)$ where $\mu_{i j}$ indicates the degree to which the object $x_{i}$ is preferred to the object $x_{j}, v_{i j}$ denotes the degree to which the object $x_{i}$ is not preferred to the object $x_{j}$, and $\pi_{i j}=\sqrt{1-\mu_{i j}^{2}-v_{i j}^{2}}$ is interpreted as a hesitancy degree, with the conditions:

$$
\mu_{i j}, v_{i j} \in[0,1], \mu_{i j}^{2}+v_{i j}^{2} \leq 1, \mu_{i j}=v_{j i}, \mu_{i i}=v_{i i}=0.5, \text { for all } i, j=1,2, \ldots, n \text {. }
$$

\subsection{Evaluation of Hospitals}

For a decision-making problem involving the evaluation of five hospitals $x_{i}, i=1,2, \ldots, 5$ of a city, assume that the decision maker compares the hospital $x_{1}$ with all the other hospitals $x_{i}, i=2,3,4,5$ under the criterion 'facilities', and provides the judgements by using a directed network of the PFPR whose vertices represent the hospitals as shown in Figure 11.

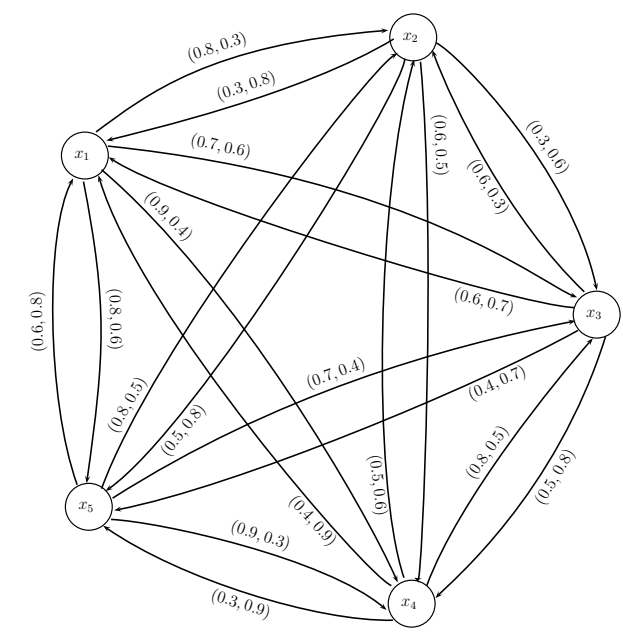

Figure 11. Directed network of the Pythagorean fuzzy preference relation (PFPR).

The corresponding adjacent matrix $R$ is as follows:

$$
R=\left(r_{i j}\right)_{5 \times 5}=\left[\begin{array}{rrrrr}
(0.5,0.5) & (0.8,0.3) & (0.7,0.6) & (0.9,0.4) & (0.8,0.6) \\
(0.3,0.8) & (0.5,0.5) & (0.3,0.6) & (0.6,0.5) & (0.5,0.8) \\
(0.6,0.7) & (0.6,0.3) & (0.5,0.5) & (0.5,0.8) & (0.4,0.7) \\
(0.4,0.9) & (0.5,0.6) & (0.8,0.5) & (0.5,0.5) & (0.3,0.9) \\
(0.6,0.8) & (0.8,0.5) & (0.7,0.4) & (0.9,0.3) & (0.5,0.5)
\end{array}\right]
$$

Using Pythagorean fuzzy weighted averaging (PFWA) operator [6],

$$
r_{i}=\operatorname{PFWA}\left(r_{i 1}, r_{i 2}, \ldots, r_{i n}\right)=\left(\sqrt{1-\left(\prod_{j=1}^{n}\left(1-\mu_{i j}^{2}\right)\right)^{1 / n}},\left(\prod_{j=1}^{n} v_{i j}\right)^{1 / n}\right), \quad i=1,2,3, \ldots, n,
$$


we aggregate all $r_{i j}, j=1,2, \ldots, 5$ corresponding to the hospital $x_{i}$, and then get the complex PFN $r_{i}$ of the hospital $x_{i}$, over all the other hospitals:

$$
\begin{aligned}
& r_{1}=(0.7788,0.4644), r_{2}=(0.4637,0.6258), r_{3}=(0.5292,0.5674), \\
& r_{4}=(0.5580,0.6560), r_{5}=(0.7475,0.4743) .
\end{aligned}
$$

Utilize the score function $s\left(r_{i}\right)=\mu_{i}^{2}-v_{i}^{2}$ [7] in order to calculate the scores of $r_{i}, i=1,2, \ldots, 5$ :

$$
s\left(r_{1}\right)=0.3909, s\left(r_{2}\right)=-0.1766, s\left(r_{3}\right)=-0.0419, s\left(r_{4}\right)=-0.1190, s\left(r_{5}\right)=0.3338 \text {. }
$$

According to $s\left(r_{i}\right), i=1,2, \ldots, 5$, we get the ranking of the hospitals $x_{i}, i=1,2, \ldots, 5$ as:

$$
x_{1} \succ x_{5} \succ x_{3} \succ x_{4} \succ x_{2} \text { (where " } \succ \text { " represents "be superior to"). }
$$

Therefore, the best hospital is $x_{1}$.

Now, using Pythagorean fuzzy weighted geometric (PFWG) operator [6],

$$
r_{i}=\operatorname{PFWG}\left(r_{i 1}, r_{i 2}, \ldots, r_{i n}\right)=\left(\left(\prod_{j=1}^{n} \mu_{i j}\right)^{1 / n}, \sqrt{1-\left(\prod_{j=1}^{n}\left(1-v_{i j}^{2}\right)\right)^{1 / n}}, \quad i=1,2,3, \ldots, n,\right.
$$

we aggregate all $r_{i j}, j=1,2, \ldots, 5$ corresponding to the hospital $x_{i}$, and then get the complex PFN $r_{i}$ of the hospital $x_{i}$, over all the other hospitals:

$$
\begin{aligned}
& r_{1}=(0.7259,0.5016), r_{2}=(0.4227,0.6770), r_{3}=(0.5144,0.6505), \\
& r_{4}=(0.4743,0.7619), r_{5}=(0.6853,0.5580) .
\end{aligned}
$$

Utilize the score function to calculate the scores $s\left(r_{i}\right), i=1,2, \ldots, 5$ of $r_{i}, i=1,2, \ldots, 5$ :

$$
s\left(r_{1}\right)=0.2753, s\left(r_{2}\right)=-0.2797, s\left(r_{3}\right)=-0.1585, s\left(r_{4}\right)=-0.3555, s\left(r_{5}\right)=0.1583 .
$$

According to $s\left(r_{i}\right), i=1,2, \ldots, 5$, we get the ranking of the hospitals $x_{i}, i=1,2, \ldots, 5$ as:

$$
x_{1} \succ x_{5} \succ x_{3} \succ x_{2} \succ x_{4} .
$$

Therefore, the best hospital is $x_{1}$ again.

\subsection{Partner Selection in Supply Chain Management}

Consider a problem regarding the selection of critical factors used to assess the potential partners of a company. Supply chain management depends on strategic relationships between companies related to a supply chain. By effective coordination, companies benefit from lower cost, lower inventory levels, information sharing and thus stronger competitive edge. Many factors may impact the coordination of companies. Among them, the following is the list of four critical factors [26]:

$C_{1}:$ Response time and supply capacity;

$C_{2}$ : Quality and technical skills;

$C_{3}$ : Price and cost;

$C_{4}$ : Service level.

In order to rank the above four factors $C_{i}(\mathrm{i}=1,2,3,4)$, a committee of three decision makers $e_{k}(k=1,2,3)$ is invited. The decision makers compare each pair of these factors and provide Pythagorean fuzzy preferences contained in the PFPRs $R_{k}=\left(r_{i j}^{(k)}\right)_{4 \times 4}(k=1,2,3)$, respectively: 


$$
\begin{aligned}
& R_{1}=\left[\begin{array}{llll}
(0.5,0.5) & (0.6,0.5) & (0.9,0.3) & (0.7,0.4) \\
(0.5,0.6) & (0.5,0.5) & (0.3,0.8) & (0.8,0.5) \\
(0.3,0.9) & (0.8,0.3) & (0.5,0.5) & (0.1,0.9) \\
(0.4,0.7) & (0.5,0.8) & (0.9,0.1) & (0.5,0.5)
\end{array}\right], \\
& R_{2}=\left[\begin{array}{llll}
(0.5,0.5) & (0.3,0.9) & (0.7,0.6) & (0.5,0.8) \\
(0.9,0.3) & (0.5,0.5) & (0.7,0.4) & (0.1,0.7) \\
(0.6,0.7) & (0.4,0.7) & (0.5,0.5) & (0.3,0.6) \\
(0.8,0.5) & (0.7,0.1) & (0.6,0.3) & (0.5,0.5)
\end{array}\right], \\
& R_{3}=\left[\begin{array}{llll}
(0.5,0.5) & (0.4,0.9) & (0.6,0.5) & (0.7,0.4) \\
(0.9,0.4) & (0.5,0.5) & (0.7,0.3) & (0.2,0.6) \\
(0.5,0.6) & (0.3,0.7) & (0.5,0.5) & (0.8,0.4) \\
(0.4,0.7) & (0.6,0.2) & (0.4,0.8) & (0.5,0.5)
\end{array}\right] .
\end{aligned}
$$

Utilizing the PFWA operator,

$$
\operatorname{PFWA}\left(r_{i j}^{(1)}, r_{i j}^{(2)}, \ldots, r_{i j}^{(s)}\right)=\left(\sqrt{1-\left(\prod_{k=1}^{s}\left(1-\left(\mu_{i j}^{2}\right)(k)\right)\right)^{1 / s}},\left(\prod_{k=1}^{s}\left(v_{i j}\right)^{(k)}\right)^{1 / s}\right),
$$

we can aggregate three PFPRs into group one:

$$
R=\left[\begin{array}{cccc}
(0.50,0.50) & (0.46,0.74) & (0.77,0.45) & (0.65,0.50) \\
(0.84,0.42) & (0.50,0.50) & (0.62,0.46) & (0.55,0.59) \\
(0.49,0.72) & (0.59,0.53) & (0.50,0.50) & (0.56,0.60) \\
(0.61,0.63) & (0.61,0.25) & (0.73,0.29) & (0.50,0.50)
\end{array}\right]
$$

Draw a directed network (shown in Figure 12) corresponding to a collective PFPR above. Then, under the condition $\mu_{i j} \geq 0.5(i, j=1,2,3,4)$, a partial diagram is drawn, as shown in Figure 13.

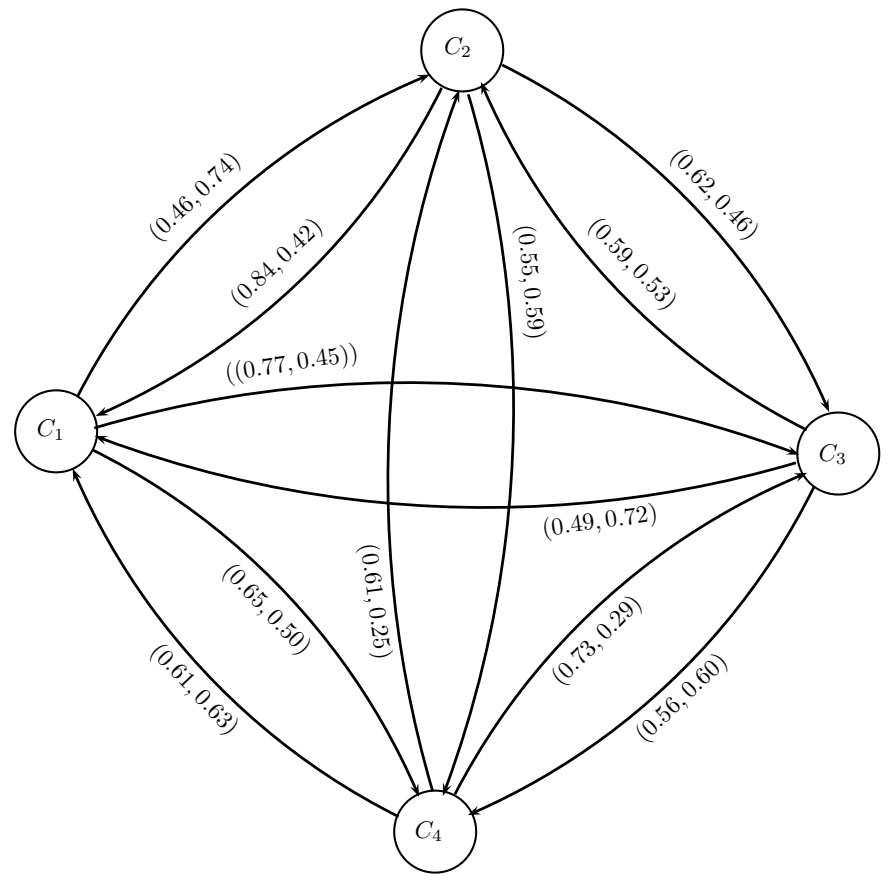

Figure 12. Directed network of the fused PFPR. 


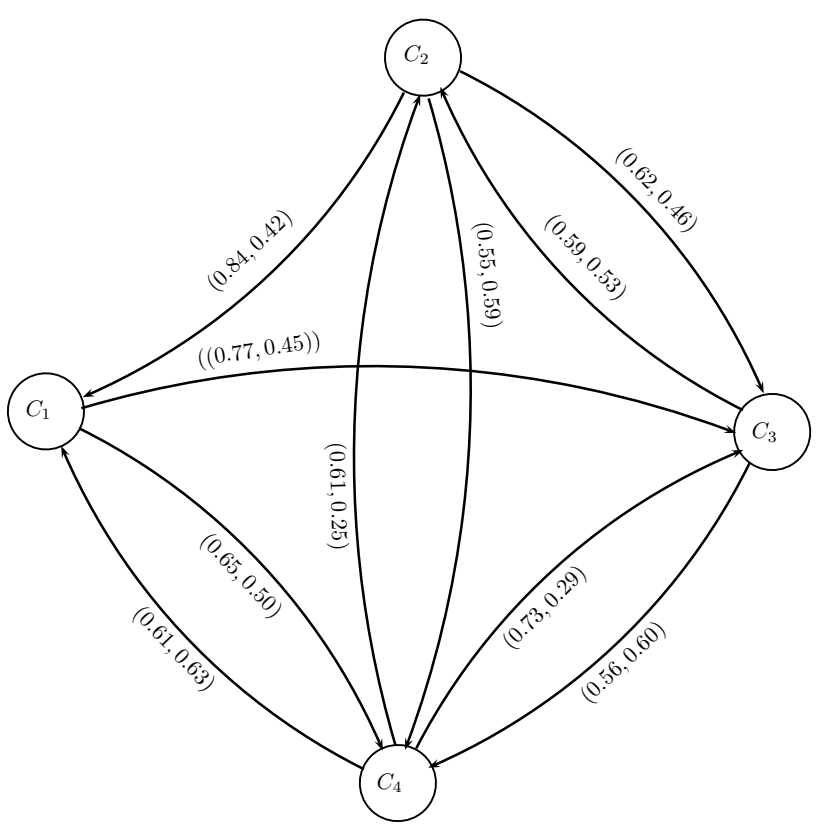

Figure 13. Partial directed network of the fused PFPR.

Calculate the out-degrees out-d $\left(C_{i}\right)(i=1,2,3,4)$ of all criteria in a partial directed network as follows:

$$
\begin{aligned}
& \text { out-d }\left(C_{1}\right)=(1.42,0.95), \text { out-d }\left(C_{2}\right)=(2.01,1.47), \\
& \text { out-d }\left(C_{3}\right)=(1.15,1.13), \text { out-d }\left(C_{4}\right)=(1.95,1.17) .
\end{aligned}
$$

According to membership degrees of out- $\mathrm{d}\left(C_{i}\right)(i=1,2,3,4)$, we get the ranking of the factors $C_{i}(i=1,2,3,4)$ as:

$$
C_{2} \succ C_{4} \succ C_{1} \succ C_{3} \text {. }
$$

Therefore, the most influential factor is quality and technical skills $C_{2}$.

Now, utilizing the PFWG operator,

$$
\operatorname{PFWG}\left(r_{i j}^{(1)}, r_{i j}^{(2)}, \ldots, r_{i j}^{(s)}\right)=\left(\left(\prod_{k=1}^{s}\left(\mu_{i j}\right)^{(k)}\right)^{1 / s}, \sqrt{1-\left(\prod_{k=1}^{s}\left(1-\left(v_{i j}^{2}\right)^{(k)}\right)\right)^{1 / s}}\right),
$$

we can aggregate three PFPRs into group one:

$$
R=\left[\begin{array}{llll}
(0.50,0.50) & (0.42,0.84) & (0.72,0.49) & (0.63,0.61) \\
(0.74,0.46) & (0.50,0.50) & (0.53,0.59) & (0.25,0.61) \\
(0.45,0.77) & (0.46,0.62) & (0.50,0.50) & (0.29,0.73) \\
(0.50,0.65) & (0.59,0.55) & (0.60,0.56) & (0.50,0.50)
\end{array}\right]
$$

According to the fused PFPR above, draw the directed network as shown in Figure 14. We select those PFNs whose membership degrees $\mu_{i j} \geq 0.5(i, j=1,2,3,4)$ (see Figure 15). Calculate the out-degrees out-d $\left(C_{i}\right)$ of all criteria as follows:

out-d $\left(C_{1}\right)=(1.35,1.1)$, out-d $\left(C_{2}\right)=(1.27,1.05)$, out-d $\left(C_{3}\right)=(0,0)$, out-d $\left(C_{4}\right)=(1.69,1.76)$.

According to membership degrees of out- $\mathrm{d}\left(C_{i}\right)(i=1,2,3,4)$, we get the ranking of the factors $C_{i}(i=1,2,3,4)$ as:

$$
C_{4} \succ C_{1} \succ C_{2} \succ C_{3} \text {. }
$$


Therefore, the service level is the most influential factor.

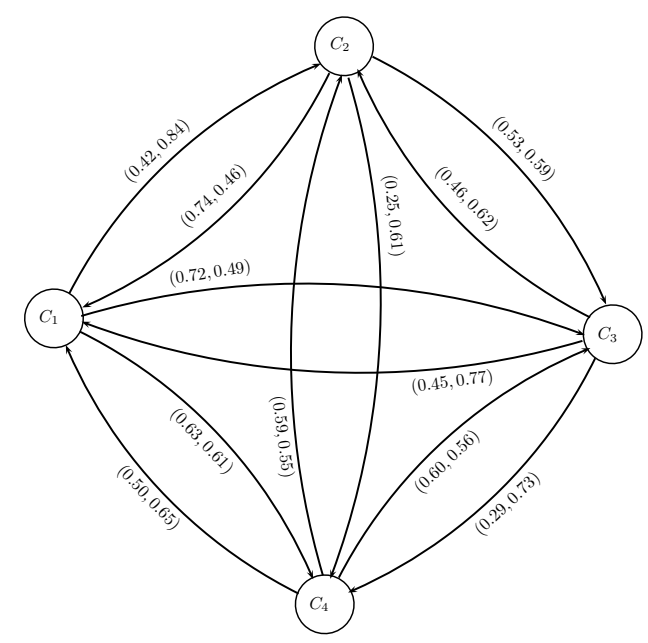

Figure 14. Directed network of the fused PFPR.

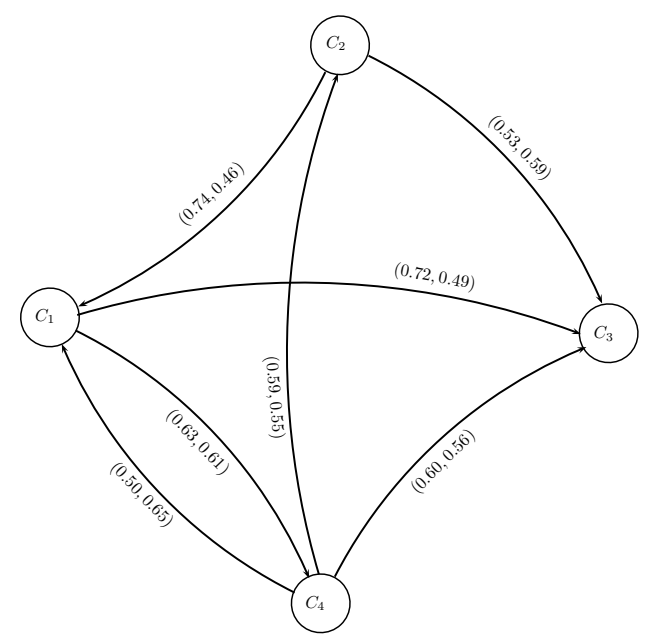

Figure 15. Partial directed network of the fused PFPR.

\subsection{Electronic Learning Main Factors Evaluation}

The electronic learning (e-learning) not only can provide expediency for learners to study courses and professional knowledge without the constraint of time and space especially in an asynchronous distance e-learning system, but also may save internal training cost for some enterprises' organizations in a long-term strategy. The e-learning becomes more and more popular along with the advancement of information technology and has played an important role in teaching and learning not only in different levels of schools but also in various commercial or industrial companies. Many schools and businesses invest manpower and money in e-learning to enhance their hardware facilities and software contents. Thus, it is meaningful and urgent to determine which is the most important among the main factors that influence the e-learning effectiveness. There are five key factors (or criteria) to evaluate the effectiveness of an e-learning system [27]:

$C_{1}$ : he synchronous learning;

$C_{2}$ : the e-learning material;

$C_{3}$ : he quality of web learning platform;

$C_{4}$ : e-learning course flexibility;

$C_{5}$ : the self-learning. 
In order to rank the above five factors, a committee comprising four decision makers $e_{k}(k=1,2,3,4)$ (whose weight vector is $w=\left(\frac{1}{4}, \frac{1}{4}, \frac{1}{4}, \frac{1}{4}\right)$ ) is founded. Based on their experiences, the decision makers compare each pair of factors (or criteria) $C_{i}(i=1,2,3,4,5)$ and give individual judgments using the following PFPRs $R_{k}=\left(r_{i j}^{(k)}\right)_{5 \times 5}(k=1,2,3,4)$ :

$$
\begin{aligned}
& R_{1}=\left[\begin{array}{lllll}
(0.5,0.5) & (0.8,0.6) & (0.7,0.5) & (0.4,0.7) & (0.1,0.8) \\
(0.6,0.8) & (0.5,0.5) & (0.4,0.5) & (0.5,0.6) & (0.3,0.5) \\
(0.5,0.7) & (0.5,0.4) & (0.5,0.5) & (0.7,0.6) & (0.6,0.7) \\
(0.7,0.4) & (0.6,0.5) & (0.6,0.7) & (0.5,0.5) & (0.4,0.8) \\
(0.8,0.1) & (0.5,0.3) & (0.7,0.6) & (0.8,0.4) & (0.5,0.5)
\end{array}\right], \\
& R_{2}=\left[\begin{array}{lllll}
(0.5,0.5) & (0.2,0.7) & (0.4,0.8) & (0.6,0.7) & (0.3,0.7) \\
(0.7,0.2) & (0.5,0.5) & (0.7,0.5) & (0.3,0.8) & (0.4,0.5) \\
(0.8,0.4) & (0.5,0.7) & (0.5,0.5) & (0.6,0.4) & (0.6,0.7) \\
(0.7,0.6) & (0.8,0.3) & (0.4,0.6) & (0.5,0.5) & (0.3,0.5) \\
(0.7,0.3) & (0.5,0.4) & (0.7,0.6) & (0.5,0.3) & (0.5,0.5)
\end{array}\right], \\
& R_{3}=\left[\begin{array}{lllll}
(0.5,0.5) & (0.7,0.6) & (0.3,0.8) & (0.4,0.6) & (0.2,0.7) \\
(0.6,0.7) & (0.5,0.5) & (0.4,0.7) & (0.8,0.1) & (0.6,0.5) \\
(0.8,0.3) & (0.7,0.4) & (0.5,0.5) & (0.7,0.5) & (0.4,0.7) \\
(0.6,0.4) & (0.1,0.8) & (0.5,0.7) & (0.5,0.5) & (0.3,0.5) \\
(0.7,0.2) & (0.5,0.6) & (0.7,0.4) & (0.5,0.3) & (0.5,0.5)
\end{array}\right], \\
& R_{4}=\left[\begin{array}{lllll}
(0.5,0.5) & (0.6,0.8) & (0.1,0.7) & (0.3,0.8) & (0.7,0.4) \\
(0.8,0.6) & (0.5,0.5) & (0.3,0.5) & (0.3,0.4) & (0.6,0.7) \\
(0.7,0.1) & (0.5,0.3) & (0.5,0.5) & (0.2,0.9) & (0.3,0.5) \\
(0.8,0.3) & (0.4,0.3) & (0.9,0.2) & (0.5,0.5) & (0.4,0.8) \\
(0.4,0.7) & (0.7,0.6) & (0.5,0.3) & (0.8,0.4) & (0.5,0.5)
\end{array}\right] .
\end{aligned}
$$

Utilize the aggregation operator to fuse all the individual PFPRs $R_{k}=\left(r_{i j}^{(k)}\right)_{5 \times 5}(k=1,2,3,4)$ into the collective PFPR $R=\left(r_{i j}\right)_{5 \times 5}$. Here, we apply the PFWA operator to fuse the individual PFPR. Thus, we have

$$
\begin{gathered}
\operatorname{PFWA}\left(r_{i j}^{(1)}, r_{i j}^{(2)}, \ldots, r_{i j}^{(s)}\right)=\left(\sqrt{1-\left(\prod_{k=1}^{s}\left(1-\left(\mu_{i j}^{2}\right)(k)\right)\right)^{1 / s}},\left(\prod_{k=1}^{s}\left(v_{i j}\right)^{(k)}\right)^{1 / s}\right), \\
R=\left[\begin{array}{ccccc}
(0.50,0.50) & (0.65,0.67) & (0.46,0.69) & (0.45,0.70) & (0.43,0.63) \\
(0.69,0.51) & (0.50,0.50) & (0.49,0.54) & (0.56,0.37) & (0.50,0.54) \\
(0.73,0.30) & (0.56,0.43) & (0.50,0.50) & (0.61,0.57) & (0.50,0.64) \\
(0.71,0.41) & (0.58,0.44) & (0.69,0.49) & (0.50,0.50) & (0.35,0.63) \\
(0.69,0.25) & (0.56,0.46) & (0.66,0.46) & (0.69,0.35) & (0.50,0.50)
\end{array}\right] .
\end{gathered}
$$

Draw a directed network corresponding to a collective PFPR above, as shown in Figure 16. Then, under the condition $\mu_{i j} \geq 0.5(i, j=1,2,3,4,5)$, a partial diagram is drawn, as shown in Figure 17. 


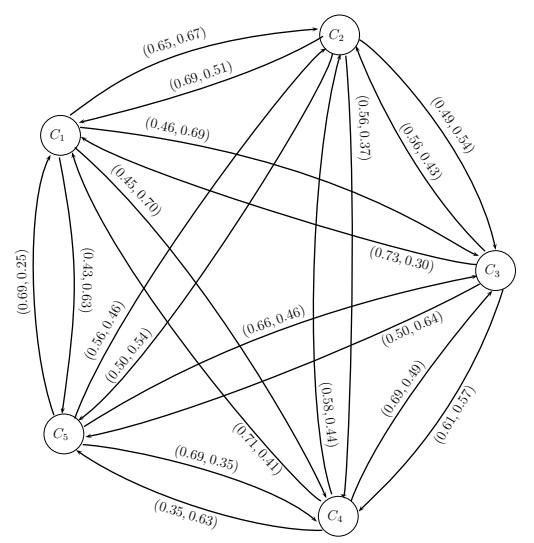

Figure 16. Directed network of the fused PFPR.

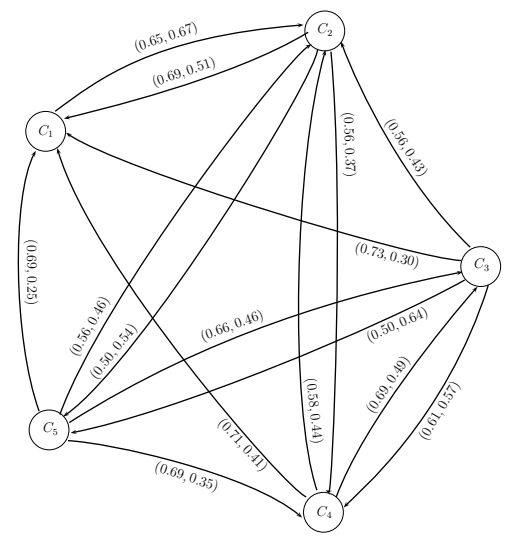

Figure 17. Partial directed network of the fused PFPR.

Calculate the out-degrees out-d $\left(C_{i}\right)(i=1,2,3,4,5)$ of all criteria in a partial directed network as follows:

$$
\begin{aligned}
& \text { out-d }\left(C_{1}\right)=(0.65,0.67), \text { out-d }\left(C_{2}\right)=(1.75,1.42), \text { out-d }\left(C_{3}\right)=(2.4,1.94), \\
& \text { out-d }\left(C_{4}\right)=(1.98,1.34), \text { out-d }\left(C_{5}\right)=(2.6,1.52) .
\end{aligned}
$$

According to membership degrees of out-d $\left(C_{i}\right)(i=1,2,3,4,5)$, we get the ranking of the factors $C_{i}(i=1,2,3,4,5)$ as:

$$
C_{5} \succ C_{3} \succ C_{4} \succ C_{2} \succ C_{1} .
$$

Therefore, the self-learning is the most influential factor.

Now, utilizing the PFWG operator,

$$
\operatorname{PFWG}\left(r_{i j}^{(1)}, r_{i j}^{(2)}, \ldots, r_{i j}^{(s)}\right)=\left(\left(\prod_{k=1}^{s}\left(\mu_{i j}\right)^{(k)}\right)^{1 / s}, \sqrt{1-\left(\prod_{k=1}^{s}\left(1-\left(v_{i j}^{2}\right)^{(k)}\right)\right)^{1 / s}}\right),
$$

we can aggregate four PFPRs into group one:

$$
R=\left[\begin{array}{ccccc}
(0.50,0.50) & (0.51,0.69) & (0.30,0.73) & (0.41,0.71) & (0.25,0.69) \\
(0.67,0.65) & (0.50,0.50) & (0.43,0.56) & (0.44,0.58) & (0.46,0.56) \\
(0.69,0.46) & (0.54,0.49) & (0.50,0.50) & (0.49,0.69) & (0.46,0.66) \\
(0.70,0.45) & (0.37,0.56) & (0.57,0.61) & (0.50,0.50) & (0.35,0.69) \\
(0.63,0.43) & (0.54,0.50) & (0.64,0.50) & (0.63,0.35) & (0.50,0.50)
\end{array}\right]
$$


According to the fused PFPR above, draw the directed network as shown in Figure 18. We select those PFNs whose membership degrees $\mu_{i j} \geq 0.5(i, j=1,2,3,4,5)$ (see Figure 19).

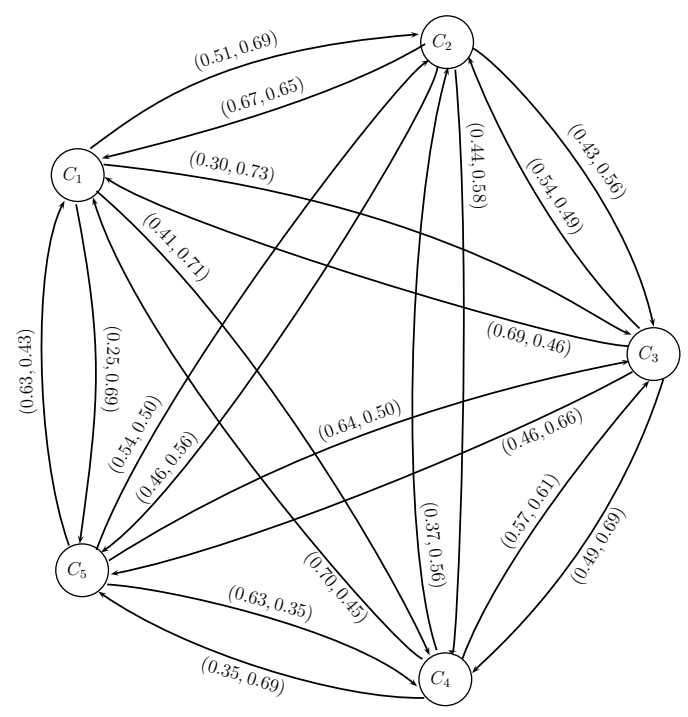

Figure 18. Directed network of the fused PFPR.

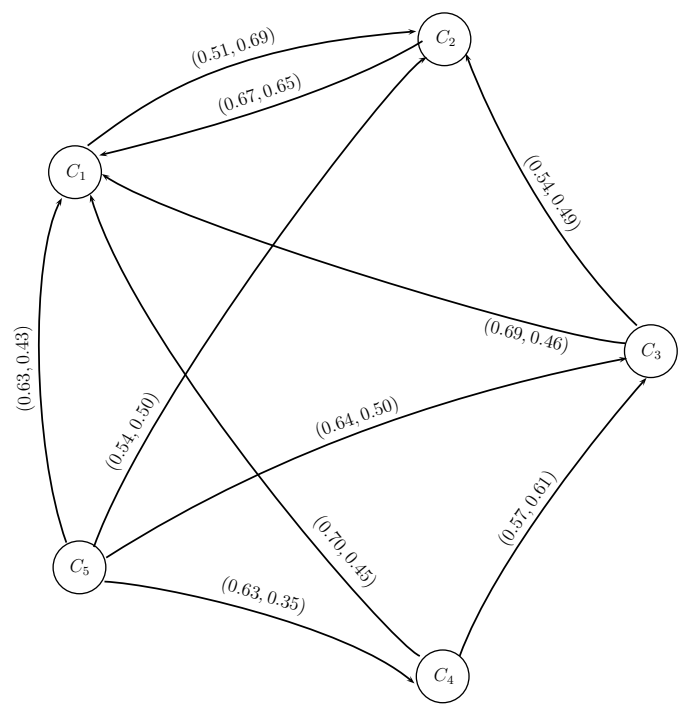

Figure 19. Partial directed network of the fused PFPR.

Calculate the out-degrees out-d $\left(C_{i}\right)$ of all criteria as follows:

$$
\begin{aligned}
& \text { out-d }\left(C_{1}\right)=(0.51,0.69), \text { out-d }\left(C_{2}\right)=(0.67,0.65), \text { out-d }\left(C_{3}\right)=(1.23,0.95), \\
& \text { out-d }\left(C_{4}\right)=(1.27,1.06), \text { out-d }\left(C_{5}\right)=(2.44,1.78) .
\end{aligned}
$$

According to membership degrees of out- $\mathrm{d}\left(C_{i}\right)(i=1,2,3,4,5)$, we get the ranking of the factors $C_{i}(i=1,2,3,4,5)$ as:

$$
C_{5} \succ C_{4} \succ C_{3} \succ C_{2} \succ C_{1} .
$$

Therefore, the self-learning is the most influential factor. 


\subsection{Comparison with IFSs}

In this sub-section, the application example concerning the evaluation of hospitals is compared with IFSs, in order to present the novelty of the introduced approach.

To present a comparison with IFS methods, firstly, we check the constraint condition $0 \leq \mu_{\alpha}+v_{\alpha} \leq 1$ for the decision matrix of preference relations, as shown in Table 1.

Table 1. Checking intuitionistic fuzzy sets (IFSs) requirement to address the problem.

\begin{tabular}{|c|c|c|c|c|c|}
\hline$R$ & $x_{1}$ & $x_{2}$ & $x_{3}$ & $x_{4}$ & $x_{5}$ \\
\hline$x_{1}$ & $0.5+0.5=1$ & $0.8+0.3>1$ & $0.7+0.6>1$ & $0.9+0.4>1$ & $0.8+0.6>1$ \\
\hline$x_{2}$ & $0.3+0.8>1$ & $0.5+0.5=1$ & $0.3+0.6<1$ & $0.6+0.5>1$ & $0.5+0.8>1$ \\
\hline$x_{3}$ & $0.6+0.7>1$ & $0.6+0.3<1$ & $0.5+0.5=1$ & $0.5+0.8>1$ & $0.4+0.7>1$ \\
\hline$x_{4}$ & $0.4+0.9>1$ & $0.5+0.6>1$ & $0.8+0.5>1$ & $0.5+0.5=1$ & $0.3+0.9>1$ \\
\hline$x_{5}$ & $0.6+0.8>1$ & $0.8+0.5>1$ & $0.7+0.4>1$ & $0.9+0.3>1$ & $0.5+0.5=1$ \\
\hline
\end{tabular}

The bold values represent values that do not satisfy the mentioned constraint. From Table 1, it can be observed that the space in which a number can be defined in IFSs is reduced in comparison with PFSs. Since the values of experts' judgments are not in the space of IFS, it is not possible to use the exact values to IFSs methods. Therefore, it is concluded that using PFS increases the flexibility and power of the experts in expressing their judgments and, while considering the limited space of IFSs in comparison with PFSs, it is not possible to solve the case presented in this paper by using IFSs. Similarly, the other application examples concerning the partner selection in supply chain management and the electronic learning main factors evaluation can be compared with IFSs to show the superiorities of the introduced approach.

\section{Conclusions}

A fuzzy graph can well describe the uncertainty of all kinds of networks. Pythagorean fuzzy models give more precision, flexibility and compatibility to the system as compared to the classical, fuzzy and intuitionistic fuzzy models. In this paper, we have introduced a new concept of PFGs. We have developed a series of operational laws of PFGs and investigated their desirable properties in detail. We have determined the degree and total degree of a vertex in PFGs formed by these operations in terms of the degree of vertices in the given PFGs in some particular cases. We have proposed the concept of PFPRs. Finally, applications of PFG theory in decision-making based on PFPRs are presented to illustrate the applicability of the proposed generalization of fuzzy graph theory. We are extending our research work to (1) Interval-valued Pythagorean fuzzy graphs; (2) Simplified interval-valued Pythagorean fuzzy graphs; and (3) Hesitant Pythagorean fuzzy graphs.

Author Contributions: S.N., S.A. and M.A. conceived and designed the experiments; S.N. wrote the paper.

Conflicts of Interest: The authors declare that they have no conflict of interest regarding the publication of the research article.

\section{References}

1. Zadeh, L.A. Fuzzy sets. Inf. Control 1965, 8, 338-353. [CrossRef]

2. Atanassov, K.T. Intuitionistic Fuzzy Sets, VII ITKR's Session, Sofia, Deposed in Central Sci; 1697/84; Central Library of the Bulgarian Academy of Sciences: Sofia, Bulgaria, 1983. (In Bulgarian)

3. Xu, Z. Intuitionistic fuzzy aggregation operators. IEEE Trans. Fuzzy Syst. 2007, 15, 1179-1187.

4. Yager, R.R. Pythagorean fuzzy subsets. In Proceedings of the Joint IFSA World Congress and NAFIPS Annual Meeting, Edmonton, AB, Canada, 24-28 June 2013; pp. 57-61.

5. Yager, R.R.; Abbasov, A.M. Pythagorean membership grades, complex numbers, and decision making. Int. J. Intell. Syst. 2013, 28, 436-452. [CrossRef] 
6. Yager, R.R. Pythagorean membership grades in multi-criteria decision making. IEEE Trans. Fuzzy Syst. 2014, 22, 958-965. [CrossRef]

7. Zhang, X.; Xu, Z. Extension of TOPSIS to multiple-criteria decision making with Pythagorean fuzzy sets. Int. J. Intell. Syst. 2014, 29, 1061-1078. [CrossRef]

8. Peng, X.; Yang, Y. Some results for Pythagorean fuzzy sets. Int. J. Intell. Syst. 2015, 30, 1133-1160. [CrossRef]

9. Ren, P.; Xu, Z.; Gou, X. Pythagorean fuzzy TODIM approach to multi-criteria decision making. Appl. Soft Comput. 2016, 42, 246-259. [CrossRef]

10. Kaufmann, A. Introduction a la Theorie des Sour-Ensembles Flous; Masson et Cie: Paris, French, 1973.

11. Zadeh, L.A. Similarity relations and fuzzy orderings. Inf. Sci. 1971, 3, 177-200. [CrossRef]

12. Rosenfeld, A. Fuzzy Graphs, Fuzzy Sets and Their Applications; Zadeh, L.A., Fu, K.S., Shimura, M., Eds.; Academic Press: New York, NY, USA, 1975; pp. 77-95.

13. Mordeson, J.N.; Peng, C.S. Operations on fuzzy graphs. Inf. Sci. 1994, 79, 159-170. [CrossRef]

14. Gani, A.N.; Radha, K. The degree of a vertex in some fuzzy graphs. Int. J. Algorith. Comput. Math. 2009, 2, $107-116$.

15. Nirmala, G.; Vijaya, M. Fuzzy graphs on composition, tensor and normal products. Int. J. Sci. Res. Publ. 2012, $2,1-7$.

16. Akram, M.; Davvaz, B. Strong intuitionistic fuzzy graphs. Filomat 2012, 26, 177-196. [CrossRef]

17. Parvathi, R.; Karunambigai, M.G. Intuitionistic fuzzy graphs. In Computational Intelligence, Theory and Applications; Springer: Berlin/Heidelberg, Germany, 2006; pp. 139-150.

18. Sarwar, M.; Akram, M. An algorithm for computing certain metrics in intuitionistic fuzzy graphs. J. Intell. Fuzzy Syst. 2016, 30, 2405-2416. [CrossRef]

19. Ashraf, S.; Naz, S.; Rashmanlou, H.; Malik, M.A. Regularity of graphs in single valued neutrosophic environment. J. Intell. Fuzzy Syst. 2017, 33, 529-542. [CrossRef]

20. Naz, S.; Rashmanlou, H.; Malik, M.A. Operations on single valued neutrosophic graphs with application. J. Intell. Fuzzy Syst. 2017, 32, 2137-2151. [CrossRef]

21. Akram, M.; Siddique, S. Neutrosophic competition graphs with applications. J. Intell. Fuzzy Syst. 2017, 33, 921-935. [CrossRef]

22. Akram, M.; Shahzadi, S.; Smarandache, F. Multi-attribute decision-making method based on neutrosophic soft rough information. Axioms 2018, 7, 19, doi:10.3390/axioms7010019. [CrossRef]

23. Sarwar, M.; Akram, M. Representation of graphs using m-polar fuzzy environment. Ital. J. Pure Appl. Math. 2017, 38, 291-312.

24. Shahzadi, S.; Akram, M. Graphs in an intuitionistic fuzzy soft environment. Axioms 2018, 7, 20, doi:10.3390/axioms7020020. [CrossRef]

25. Zafar, F.; Akram, M. A novel decision-making method based on rough fuzzy information. Int. J. Fuzzy Syst. 2018, 20, 1000-1014. [CrossRef]

26. Chen, C.Y.; Xu, L.G. Model for selecting partners in supply chain management. Chin. J. Manag. Sci. 2001, 9,57-62.

27. Liao, H.; Xu, Z.; Xia, M. Multiplicative consistency of interval-valued intuitionistic fuzzy preference relation. J. Intell. Fuzzy Syst. 2014, 27, 2969-2985.

(c) 2018 by the authors. Licensee MDPI, Basel, Switzerland. This article is an open access article distributed under the terms and conditions of the Creative Commons Attribution (CC BY) license (http://creativecommons.org/licenses/by/4.0/). 